Universidad Politécnica de Madrid

Escuela Técnica Superior de Ingeniería Aeronáutica y del Espacio
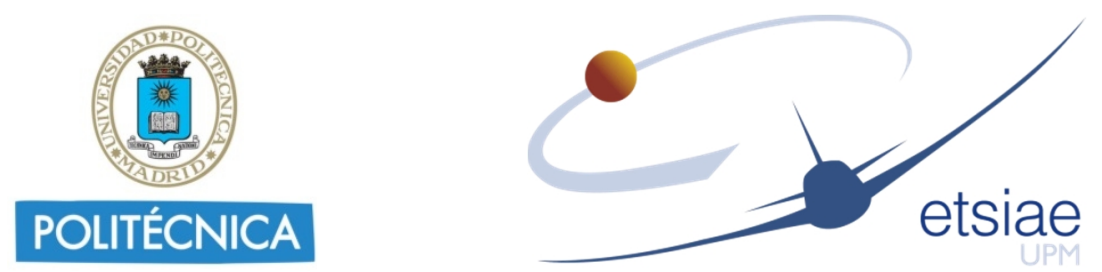

\title{
Quasi-Autonomous Spacecraft Thermal Model Reduction
}

\author{
Germán Fernández Rico \\ Ingeniero Aeronáutico
}

Directores:

Dra. Ingeniera Aeronáutica Isabel Pérez Grande

Dr. Ingeniero Aeronáutico Ángel Sanz Andrés

Madrid, 2018 

Tribunal nombrado por el Sr. Rector Magfco. de la Universidad Politécnica de Madrid, el día de. de $20 \ldots$.

Presidente:

Vocal:

Vocal:

Vocal:

Secretario:

Suplente:

Suplente:

Realizado el acto de defensa y lectura de la Tesis el día de. de $20 \ldots$ en la E.T.S.I. /Facultad

Calificación

EL PRESIDENTE

LOS VOCALES

EL SECRETARIO 



\section{Abstract}

Thermal analysis has become a core activity of the spacecraft and space subsystems design. Thermal models complexity is increasing and, for some activities, it is necessary to reduce the size of the models in order to make them easier to handle and faster to analyze (among other reasons). Thermal model reduction is a recurrent activity for space thermal analysts, thus the convenience of having a technique to reduce the models in an automatic and reliable way.

A matrix method is developed to reduce the number of elements of spacecraft thermal mathematical models based on the lumped parameter method. The aim of this method is to achieve a satisfactory thermal model reduction for steady state problems, in an automatic way, while preserving the physical meaning of the system and the main characteristics of the model. The simplicity of the method, and the computational cost, are also taken into account. The reduction process is based on the manipulation of the conductive coupling matrix, taking into account the temperature differences among the nodes of the detailed model. The resulting matrix is then treated as an undirected sparse graph adjacency matrix. Then, a depth-first search algorithm is used to find the connected components, which defines the condensed nodes. Finally, all the thermal entities are reduced, and the results from the condensed model are compared to those from the detailed one, upon definition of results correlation criteria. A brief theoretical introduction is given before describing the reduction algorithm in detail.

The entire reduction process is tested on a simple model, describing the method step-by-step procedure. Then the reduction technique is tested on a real thermal model, showing a good performance. The reduced thermal model is also tested in transient conditions, in order to check the usability of the condensed model in that kind of analysis.

In the conclusions section the characteristics and limitations of this method are shown, as well as the possible future work to be done in order to improve the method and solve its deficiencies.

Finally, the annex includes some additional material that completes what is exposed along the main body of the dissertation. 


\section{RESUMEN}

El análisis térmico constituye una parte clave del diseño de vehículos espaciales y sus diferentes subsistemas. Los modelos térmicos usados a tal efecto han ido ganando en complejidad y tamaño conforme han aumentado los recursos informáticos disponibles. A menudo, es necesario reducir el tamaño de dichos modelos, con el objetivo de facilitar su uso y acortar los tiempos de análisis, entre otras razones. La reducción de modelos térmicos es una actividad recurrente para el analista, de ahí la conveniencia de disponer de un método fiable y automático que permita obtener dichos modelos reducidos.

Para reducir el número de nodos de los modelos térmicos basados en el método de las capacitancias concentradas, se ha desarrollado un procedimiento matricial. El objetivo de dicho procedimiento es conseguir una reducción satisfactoria para problemas estacionarios, de forma automática, preservando el significado físico y las características del modelo. La simplicidad del método y su coste computacional han sido también tenidos en cuenta. El proceso de reducción está basado en la manipulación de la matriz de acoplamientos conductivos, teniendo en cuenta también la diferencia de temperatura entre los distintos nodos del modelo térmico detallado. La matriz resultante es tratada como una matriz de adyacencia que representa un grafo disperso no dirigido. Sobre esa matriz de adyacencia se usa un algoritmo de búsqueda en profundidad para encontrar los componentes conectados, que definirán los nodos condensados que forman el modelo reducido. Después, todas las matrices y vectores que conforman el modelo térmico detallado son compactados, y se pueden obtener resultados (temperaturas y flujos de calor) con el modelo reducido. Estos resultados pueden ser comparados con aquellos obtenidos con el modelo detallado, usando para ello los criterios de correlación previamente definidos. Al principio del texto se realiza una breve introducción teórica del algoritmo, describiéndose a continuación el proceso en detalle.

El método de reducción se ha probado primero en un modelo simple estacionario, describiendo el proceso paso por paso, y a continuación en un modelo térmico real en un análisis transitorio, en el que se muestra también un buen rendimiento.

En las conclusiones se recogen las principales características y limitaciones del método de reducción, así como posibles vías para mejorarlo y ampliar su ámbito de aplicación.

Finalmente, en el anexo se muestra diverso material adicional que completa lo expuesto a lo largo del texto principal de la disertación. 


\section{ACKNOWLEDGEMENTS}

I would like to thank Dr. Isabel Pérez Grande for giving me the opportunity to start my career at the Instituto de Microgravedad "Ignacio Da Riva" (IDR/UPM), introducing me into the fascinating world of space instrumentation and thermal control. I would also like to thank Dr. Ángel Sanz Andrés, for his ideas, his support and his encouragement to advance with the thesis.

I must thank my colleague and friend Ignacio Torralbo Gimeno for his ideas, the good times we shared together while struggling with ESATAN, developing new methods and scripts, and a long et cetera. Also, my extended gratitude to the rest of the team at IDR.

I thank Dr. Joachim Woch and Dr. Luca Teriaca for welcoming me at the Max Planck Institute for Solar System Research and integrating me into their teams. Also thanks to the International Max Planck Research School (IMPRS) for Solar System Science for its support.

I thank also Miguel Álvarez Copano for proofreading the first draft of this thesis dissertation.

Finally, I want to thank my parents Javier and Jazmín and my wife Lidia, for their love, patience and unconditional support.

This work has been partially supported by the Spanish Ministerio de Economía, Industria y Competitividad under projects ESP2014-56169-C6-6-R and ESP2016-77548-C5-3-R.

The author was awarded a mobility grant from Consejo Social of Universidad Politécnica de Madrid, which supported his research stay carried out at the Max Planck Institute for Solar System Research, Göttingen, Germany, from April to June 2014. 



\section{Contents}

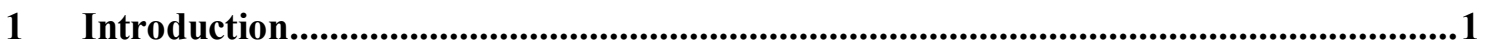

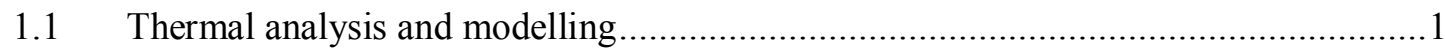

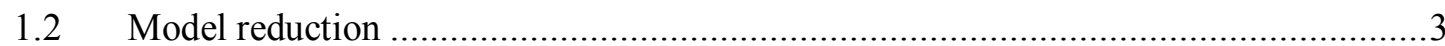

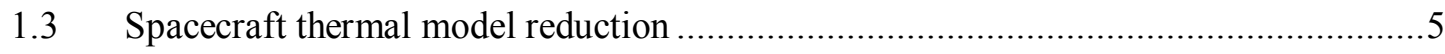

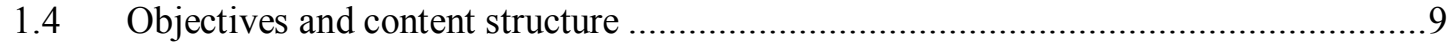

2 Theoretical background ......................................................................................................11

$2.1 \quad$ Reduction analysis of 4-node model......................................................................

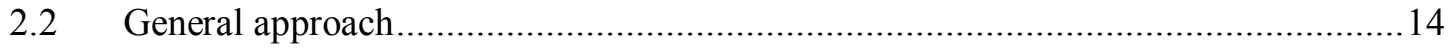

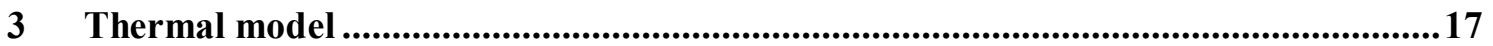

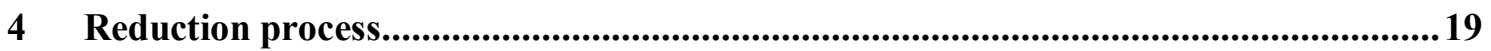

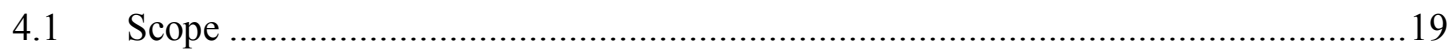

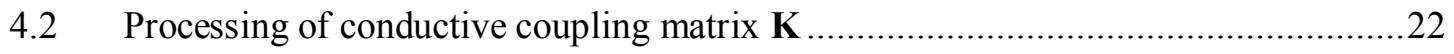

4.2.1 Building the dimensionless coupling matrix $\tilde{\mathbf{K}}$............................................22

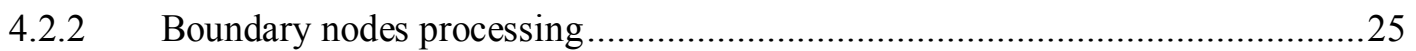

4.2.3 Filtering of the dimensionless conductive coupling matrix..............................26

4.3 Construction and process of temperature difference matrix ....................................27

4.3.1 Building and filtering the temperature difference matrix ................................27

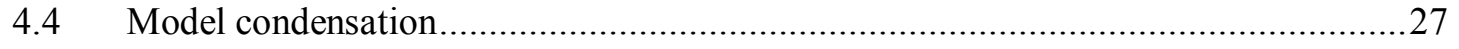

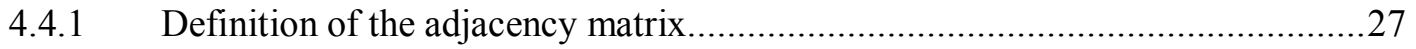

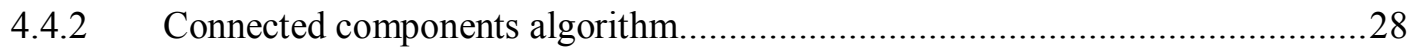

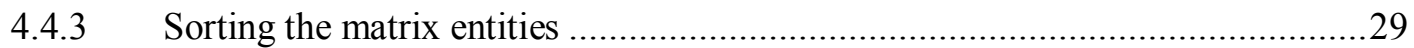

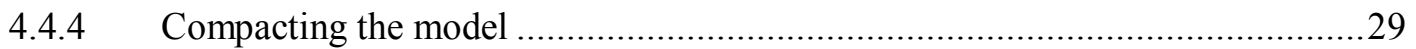

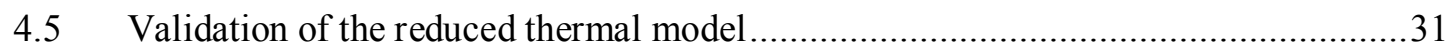

$5 \quad$ Testing of the proposed methodology …........................................................................33

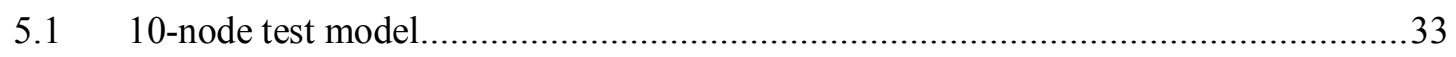

5.2 Solar Orbiter PHI Focal Plane Assembly test model ..................................................45

5.2.1 Reduction of the thermal mathematical model .................................................46

5.2.2 Reduction of the geometrical mathematical model.........................................58

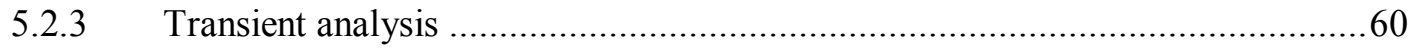

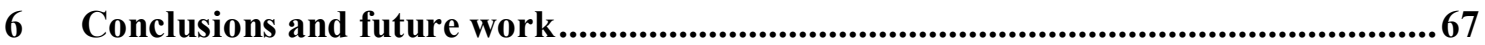




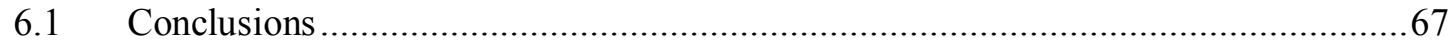

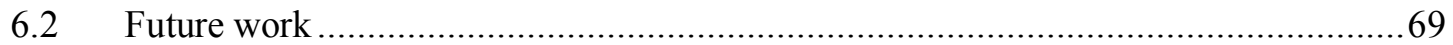

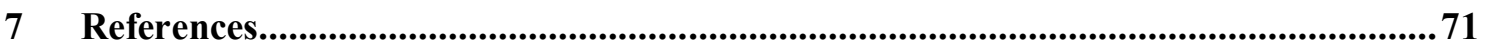

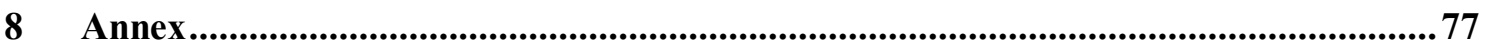

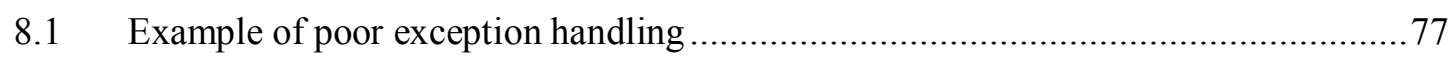

8.2 Output of the PHI OPT FPA model reduction (extract) ...........................................

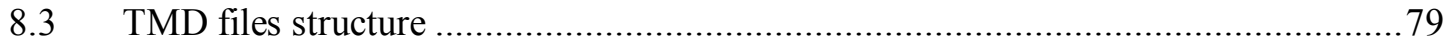

8.3.1 ESATAN-TMS produced TMD file (as of release 7 service pack 2) .................79

8.3.2 TMD file output by the reduction software ................................................... 80 


\section{LIST OF FIGURES}

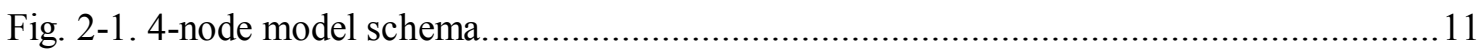

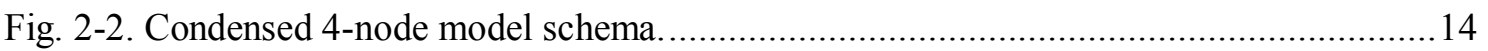

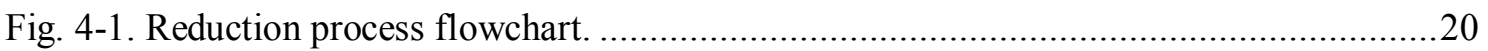

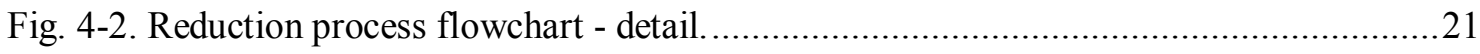

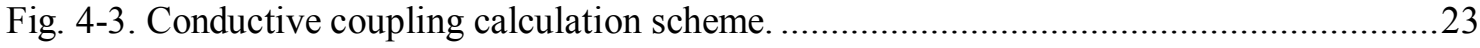

Fig. 4-4. Boundary nodes and internal boundary nodes retention process. a) Shows nodes interconnected, including boundary nodes, in orange. b) Internal boundary nodes are identified, in blue. c) Isolation of boundary and internal boundary nodes, by removing conductive couplings, in red. d) Final state of the nodes, once the nodes have been isolated. ......................26

Fig. 4-5. Example conductive couplings matrix (K) on the top left (integer values for the sake of matrix compactness), the adjacency matrix A (after binarizing $\mathrm{K}$ ) on the top right and the corresponding graph on the bottom. .28

Fig. 4-6. Connected components and couplings reduction process. a) Strong conductive couplings among the nodes (solid black lines) and weak connections (dashed black lines). b) The connected components are found within the mesh, condensing the nodes in reduced nodes. Strong couplings vanish among the condensed nodes. c) The weak couplings are summed up to find the equivalent couplings between two reduced nodes. d) Final status of the reduced model.

Fig. 5-1. 10-node model sketch (node 3 not shown).

Fig. 5-2. Sketch of 10-node model conductive couplings. There is no difference between solid black and dashed grey lines in the figure. Grey dashed lines have been used solely for visualization purposes. .34

Fig. 5-3. Sketch of 10-node model with the condensed nodes. ................................................42

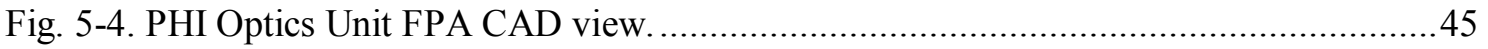

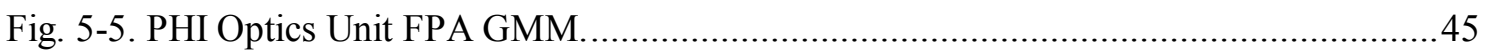

Fig. 5-6. PHI Optics Unit FPA boundary nodes (conductive)....................................................

Fig. 5-7. Sparse graphical representation of (a) matrix K and (b) matrix R.............................46

Fig. 5-8. Conductive couplings histograms of (a) matrix $\mathrm{K}$ values and (b) matrix $\mathrm{K}^{\mathrm{S}}$ values.....47

Fig. 5-9. Variation of (a) reduction ratio $r_{r}$ vs. filtering threshold $p_{f}$, and variation of (b) reduction ratio $r_{r}$ vs. temperature difference threshold $\Delta T_{\max }$. For (a), the SCC algorithm is run with $\tilde{\mathrm{K}}^{\mathrm{I}, \mathrm{F}}$. For (b), the matrix $\mathrm{K}$ is multiplied element-wise by $\Theta^{\mathrm{F}}$, and the $\mathrm{SCC}$ algorithm is run with the resulting matrix.

Fig. 5-10. Contour plot of the function $r_{r}=f\left(p_{f}, \Delta T_{\max }\right)$. Black solid lines represent constant $r_{r}$, whose value is indicated inline. Grey zone indicates successful correlation for the resulting reduced model. Orange colored zone indicates failed correlation for the resulting reduced 
model. Red line indicates the separation between both zones, being effectively the limit for a successful correlation.

Fig. 5-11. Contour plot of the function $r_{r}=f\left(p_{f} \Delta T_{\max }\right)$, zoomed at the $r_{r}=[0.5,0.9]$ zone. .....50

Fig. 5-12. Contour plot of the function $r_{r}=f\left(p_{f}, \Delta T_{\max }\right)$, zoomed at the $r_{r}=[0.5,0.9]$ zone. Orange line indicates the correlation limit line based on $\delta$. Green line indicates the limit based on $\mathrm{q}_{\mathrm{cb}}$. Blue line is for $\mathrm{q}_{\mathrm{rb}}$. Red line is the envelope of the previous three, and coincides with the red line in Fig. 5-11

Fig. 5-13. $\operatorname{Max}\{\delta\}=f\left(p_{f}, \Delta T_{\max }\right)$ over the zone $r_{r}=[0.5,0.9]$ zone. Black line: limit of the successful correlation zone in terms of temperature difference.

Fig. 5-14. $\operatorname{Max}\left\{\mathrm{q}_{\mathrm{cb}}\right\}=f\left(p_{f}, \Delta T_{\max }\right)$ over the zone $r_{r}=[0.5,0.9]$ zone. Black line: limit of the successful correlation zone in terms of conductive heat flux difference.

Fig. 5-15. $\operatorname{Max}\left\{\mathrm{q}_{\mathrm{rb}}\right\}=f\left(p_{f}, \Delta T_{\max }\right)$ over the zone $r_{r}=[0.5,0.9]$ zone. Black line: limit of the successful correlation zone in terms of radiative heat flux difference.

Fig. 5-16. (a) Variation of the reduction ratio $r_{r}$ as a function of filtering threshold $p_{f}$, keeping constant the temperature difference threshold $\Delta T_{\max }$. (b) is similar to (a), but showing $\max \left\{\delta_{i}\right\}$. (c) shows the variation of $\max \left\{q_{c b}\right\}$ as a function of $p_{f}$, again keeping constant $\Delta T_{\max }$. (d)

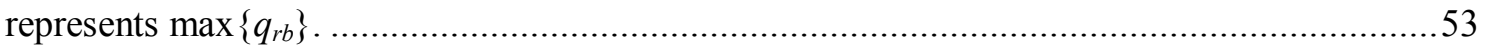

Fig. 5-17. Sparse graphical representation of restriction matrix P..........................................54

Fig. 5-18. Sparse graphical representation of (a) matrix $\mathrm{K}_{\text {sort }}$ and (b) matrix $\mathrm{R}_{\text {sort }}$. The matrices are symmetric, so the program only works with upper triangular matrices. .54

Fig. 5-19. Sparse graphical representation of (a) matrix $K^{R}$ and (b) matrix $R^{R}$. Matrix $K^{R}$ has 395 conductive couplings, whereas $\mathrm{R}^{\mathrm{R}}$ has 18 244. The matrices are symmetric, so the program only works with upper triangular matrices. .55

Fig. 5-20. Components of temperature differences vector $\delta . i$ : vector index. ..........................56

Fig. 5-21. Temperature differences histograms of (a) vector $\delta$ and (b) $\tau$. .56

Fig. 5-22. Temperature differences $\left({ }^{\circ} \mathrm{C}\right)$ between detailed and reduced nodes $(\tau)$ overlaid on the GMM .58

Fig. 5-23. Condensed node number overlaid on the FPGA GMM (top left for node range 101112 , top right for 113-124 and bottom for 125-136).....

Fig. 5-24. Evolution of the boundary nodes temperatures for transient analysis (horizontal axis time- only covers just up to $7200 \mathrm{~s}$ for more clarity).

Fig. 5-25. Evolution of the temperatures of nodes 190 and 210 (corresponding to maximum differences obtained in the transient analysis)......

Fig. 5-26. RTMM condensed node no. 190 (left) and node no. 210 (right).

Fig. 5-27. Evolution of the temperatures of nodes 4, 21, 58 and 185.

Fig. 5-28. RTMM condensed node no. 4 - housing upper lid (top left), no. 21 - cold finger (top right), no. 58 - sensor board (bottom left) and no. 185 - detector die (bottom right). 
Fig. 5-29. Components of vector $\delta$ at the end of the transient analysis

Fig. 8-1. SO/PHI Electronics Unit: variation of (a) $r_{r}$ vs. $p_{f}$, and variation of (b) $r_{r}$ vs. $\Delta T_{\max }$. For (a), the SCC algorithm is run with $\tilde{K}^{\mathrm{I}}$. F For (b), the matrix $\mathrm{K}$ is multiplied element-wise by $\Theta^{\mathrm{F}}$, and the SCC algorithm is run with the resulting matrix

\section{LIST OF TABLES}

Table 5-1. 10-node model temperature results comparison.......................................................44

Table 5-2. 10-node model heat fluxes results comparison. ...................................................... 44

Table 5-3. PHI FPA model conductive heat fluxes comparison..............................................57

Table 5-4. PHI FPA model conductive heat fluxes comparison..............................................57

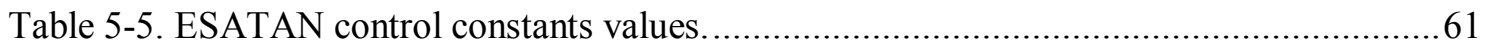

Table 5-6. PHI FPA model conductive heat fluxes comparison at the end of the transient analysis. Orange cell indicates an unfulfillment of the required heat flux difference.

Table 5-7. PHI FPA model conductive heat fluxes comparison at the end of the transient analysis 


\title{
NOMENCLATURE
}

\author{
Roman symbols
}

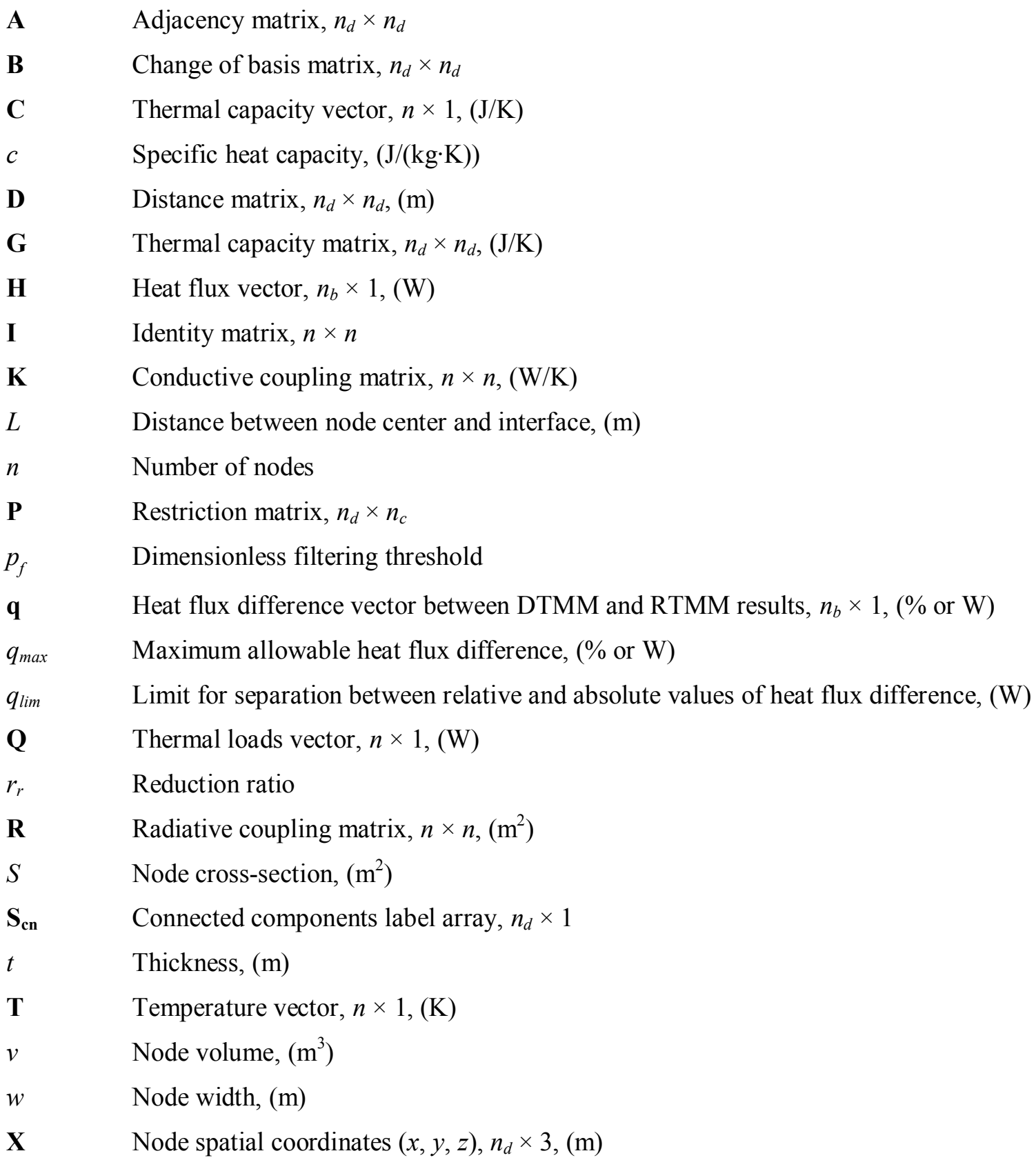




\section{Greek symbols}

$\boldsymbol{\delta} \quad$ Vector of temperature differences between derived and calculated values, $(\mathrm{K}), n_{c} \times 1$

$\delta_{\max } \quad$ Maximum allowable temperature difference, $(\mathrm{K})$

$\Delta T_{\max } \quad$ Temperature difference threshold, (K)

$\lambda \quad$ Thermal conductivity, $(\mathrm{W} /(\mathrm{m} \cdot \mathrm{K}))$

$\mu \quad$ Parameter, $\left(\mathrm{m}^{2} / \mathrm{s}\right)$

$\rho \quad$ Density, $\left(\mathrm{kg} / \mathrm{m}^{3}\right)$

$\sigma \quad$ Stefan-Boltzmann constant, $\left(\mathrm{W} /\left(\mathrm{m}^{2} \cdot \mathrm{K}^{4}\right)\right)$

$\boldsymbol{\tau} \quad$ Temp. Difference between condensed node and detailed node values, $(\mathrm{K}), n_{d} \times 1$

$\boldsymbol{\Theta} \quad$ Temperature difference matrix, $n_{d} \times n_{d},(\mathrm{~K})$

Accents

$\begin{array}{ll}- & \text { Derived values } \\ \wedge & \text { Calculated values } \\ \sim & \text { Dimensionless values }\end{array}$

Subscripts

b Boundary node

c Condensed model

$\mathrm{cb} \quad$ Conductive to boundary nodes

d Detailed model

$\max \quad$ Maximum

$\mathrm{rb} \quad$ Radiative to boundary nodes

sort Sorted

\section{Superscripts}

B Including marked boundary nodes

D Detailed model

F $\quad$ Filtered

I Including marked internal boundary nodes

R $\quad$ Reduced model

S Sizing

T Transposed

* $\quad$ Linearized 


\section{ABBREVIATIONS AND ACRONYMS}

$\begin{array}{ll}\text { AWE } & \text { Asymptotic Waveform Evaluation } \\ \text { BEM } & \text { Boundary Element Model } \\ \text { BTR } & \text { Balanced Truncation Realization } \\ \text { CAD } & \text { Computer-Aided Design } \\ \text { CDR } & \text { Critical Design Review } \\ \text { CFD } & \text { Computer Fluid Dynamics } \\ \text { CSG } & \text { Capacitance over Sum of conductors ratio } \\ \text { CSR } & \text { Compressed Storage by Rows } \\ \text { DOFs } & \text { Degrees of Freedom } \\ \text { DTIMEI } & \text { Input time step (ESATAN) } \\ \text { DTMM } & \text { Detailed Thermal Mathematical Model } \\ \text { ECSS } & \text { European Cooperation for Space Standardization } \\ \text { ESA } & \text { European Space Agency } \\ \text { FEM } & \text { Finite Element Method } \\ \text { FPA } & \text { Focal Plane Assembly } \\ \text { FPGA } & \text { Field Programmable Gate Array } \\ \text { GAM } & \text { Gravity Assisted Maneuver } \\ \text { GMM } & \text { Geometrical Mathematical Model } \\ \text { HDF } & \text { Hierarchical Data Format } \\ \text { I/F } & \text { Interface } \\ \text { LHS } & \text { Latin Hypercube Sampling } \\ \text { LPM } & \text { Lumped Parameter Method } \\ \text { MAM } & \text { Model Acceleration Method } \\ \text { MDM } & \text { Mode Displacement Method } \\ \text { MEMS } & \text { Micro-Electro-Mechanical System } \\ \text { MLI } & \text { Multi-Layer Insulation } \\ \text { MOR } & \text { Model-Order Reduction } \\ \text { NaN } & \text { Not a Number } \\ \text { ODE } & \text { Ordinary Differential Equation } \\ \text { OPT } & \text { Optics Unit } \\ \text { PHI } & \text { Polarimetric Helioseismic Imager } \\ \text { PID } & \text { Proportional-Integral-Derivative } \\ \text { Proper Orthogonal Decomposition } \\ \text { POia Lanczos }\end{array}$


REFs Radiative Exchange Factors

RGMM Reduced Geometrical Mathematical Model

RTMM Reduced Thermal Mathematical Model

SCC Strongly Connected Components

SPQ Sequential Quadratic Programming

SVD Single Value Decomposition

TAUMEL Tool for AUtomated Model Correlation using Equation Linearization

TMD Thermal Model Data

TMM Thermal Mathematical Model

TMRT Thermal Model Reduction Tool

TPWL Trajectory Piecewise Linear 



\section{INTRODUCTION}

When designing a space-borne scientific instrument or a satellite, one of the most important constraints the designer has to face are the temperature requirements of the different parts involved. Precisely, the spacecraft thermal control addresses the problem of keeping space vehicles and their components temperatures within the appropriate range [1-4]. The assessment of the compliance of the system to these thermal requirements is done through thermal analysis and testing. During the first phases of a space program (phases 0, A and B in the frame of European programs, see $[5,6])$, thermal analysis is typically the only tool for the appraisal of the system thermal performance. In later stages, thermal testing becomes fundamental for the system verification, although sometimes complete testing is not possible, due to budget or physical limitations.

\subsection{Thermal analysis and modelling}

In the earliest stages of the design, the preliminary studies can be performed using analytical calculations [7-13]. However, as the system becomes more complex and detailed, it is necessary to rely on other tools. While thermal tests allow the design to be verified (verification of the design and the thermal mathematical models), they are very complex and expensive. Complementary to the tests, thermal simulation has become more and more relevant, as the software packages have evolved rapidly in the last years. And so did the thermal models sizes: from the 400-node network used in the 1980s for Ulysses spacecraft [14] to roughly 30000 nodes used in present day Solar Orbiter [15] spacecraft thermal mathematical model (TMM).

Nowadays, it is quite usual to have very detailed thermal models for the element under study (for typical lumped parameter method (LPM) solvers, it is in the range of $10^{3}-10^{4}$ nodes). In Europe, one of the standard packages for thermal analysis in the space industry is ESATANTMS [16]).

The purpose of the thermal modelization of a system is to build up a system of ordinary differential equations (ODEs) that allows, by solving it, to find the temperatures and heat fluxes, in transient or steady state conditions. The setting up of a thermal and geometrical mathematical model starts generally with a mechanical model of the element under study. It can be a very detailed computer-aided design (CAD) model or a rough shape of the equipment. Regardless of the maturity of the mechanical design, it is necessary to build up a geometrical model, assigning to the different shapes and surfaces the corresponding thermo-optical properties -infrared emissivity, solar absorptivity- and bulk material properties -thermal conductivity, density, specific heat-. The surfaces can be meshed, depending on the detailed that is judged necessary by the engineer. Based on the type of analysis, it might be required to define orbit and mission characteristics. Before solving the thermal problem, it is necessary to calculate all the conductive couplings that are not automatically calculated by the software, as well as to implement any additional functionality or characteristic of the model (power dissipation values, thermostats, etc.). Finally, the boundary conditions, when not imposed by the orbit, must be defined. The aspect of the system of ODEs is given by

$$
C_{i} \frac{d T_{i}}{d t}=\sum_{j=1}^{n_{d}}\left[K_{i j}\left(T_{j}-T_{i}\right)+R_{i j}\left(T_{j}^{4}-T_{i}^{4}\right)\right]+Q_{i}, \quad i=1, \ldots, n_{d}, \quad i \quad j \quad,
$$


The terms of equation (1) are explained in detail in chapter 3.

If the element under study needs to be included in a higher hierarchy model, or if the analyst needs to run extensive simulations (thermal test predictions, in-orbit simulations, sensitivity analyses, etc.) the number of nodes of the model (the so-called "size"), and consequently the runtime, can become a problem. Therefore, it might be necessary to reduce the number of nodes. The reduced thermal model must behave as the detailed one (in terms of temperatures and heat fluxes through the boundaries), while having a significantly smaller number of nodes.

\section{Example}

The Solar Orbiter spacecraft detailed TMM had, at the time of the Critical Design Review (CDR), around 25000 nodes (most of them devoted to represent the platform itself, rather than the scientific instruments). The Solar Orbiter mission scientific payload consists of ten instruments, one of which is the Polarimetric and Helioseismic Imager (PHI) [17]. In turn, the PHI instrument is composed of five different units (optics, electronics, harness, and two heat rejecting entrance windows). Taking the Optics Unit for instance, its detailed TMM has around 6000 thermal nodes. The Electronics Unit thermal model, as well as other models of units belonging to different instruments, is in the same order of magnitude. This means that in case the Solar Orbiter spacecraft prime contractor wanted to assemble all the instruments detailed thermal models together with the spacecraft one, the final product would likely have more than 250000 nodes. This number might not represent a concern in structural finite element method (FEM) models, but for thermal LPM models, this number of nodes is unmanageable. Taking into account the number of analyses that are necessary for a mission such as Solar Orbiter (worst-case steady state scenarios, planetary flybys -corresponding to gravity assist maneuvers (GAM)-, off-pointing cases, etc.), such amount of nodes would make the computational effort unaffordable (let alone the difficulty of handling all the nodes and associated information, postprocessing results...). Therefore, during the whole extent of the spacecraft development, the payload teams are required to deliver representative and updated reduced thermal models of the different instrument units, so that the prime contractor can derive reliable results for the payload and their interfaces with the spacecraft, without compromising the overall size of the thermal model used to that end. Hence, some rules for delivering the reduced thermal models must be stablished. These are related to the maximum number of nodes and geometric elements within the new model, some limitations in terms of physical reduction, and the maximum acceptable temperatures and heat fluxes differences in selected analysis cases. Once the prime contractor has assembled the platform thermal model with the different reduced models from payload teams and subcontractors, new results are obtained. These can be used to derive a new set of environmental conditions, in such a way that the team responsible for the payload and the subcontractors are able to use them in order to refine their thermal models. In turn, the updated reduced thermal models are then provided back to the prime, in an iterative process that takes place during the whole mission development program.

This example reveals the importance of the reduced thermal models in spacecraft development. Before addressing the particular aspects of spacecraft thermal model reduction, let us start with a brief summary of general mathematical models reduction. 


\subsection{Model reduction}

Many references can be found in the literature related to model-order reduction (MOR). Of course, the problem of reducing the size or mathematical complexity of numerical models is not exclusive to spacecraft thermal mathematical modelling. Indeed the problem is quite the opposite: in the general MOR references, there are no examples or techniques addressed to reduced spacecraft related TMMs. MOR was originally developed in the systems and control theory disciplines, and then extended to electronic circuits, Computer Fluid Dynamics (CFD) and structural dynamics (although different authors hold different origins, see for instance [18] and [19]). One of the best reviews is given by Besselink et al in [18] (other reviews are very interesting as well, for instance [19] and [20]). Three different fields are described in that paper: structural dynamics, numerical mathematics (electronic circuits) and dynamical systems and control. Each one having different model reduction techniques.

\section{Structural dynamics}

The structural problem is mathematically modelled in terms of partial differential equations and then discretized by applying $\mathrm{FEM}^{1}$. The reduced model shall capture the global dynamic behavior of the system. According to [18], the basis for setting up reduced-order models in this field is to use a small number of free vibration modes with a reduced number of generalized coordinates, being able to represent the displacement pattern. The methods that follow this approach are called mode superposition methods. The original one is known as mode displacement method (MDM), which has some variations, like model acceleration method (MAM) and modal truncation augmentation. While these methods try to improve some aspects of the resulting reduced model, they do not provide any kind of a priori error bound, so their performance in terms of accuracy shall be evaluated after reducing the system, by comparing the results between the original and the reduced model. Alternative to the mode superposition methods, there exist the methods known as component mode synthesis techniques, such as Craig-Brampton and Rubin and MacNeal methods. The reader can consult [21] to get more information about these dynamic substructuring techniques. Besselink does not mention in [18] a well-know and popular method: the static condensation of Guyan [22] (also known as GuyanIrons). As it will be explained later in section 1.3, this is the base for a thermal model reduction method described in [23-27].

\section{Numerical mathematics}

The field of numerical mathematics corresponds mainly to electronic circuits (with large linear sub-circuits) and micro-electro-mechanical systems (MEMS). The techniques described for model reduction in this field are based in Krylov subspace methods (by transfer function expansion moment matching). This approach aims to preserve the input/output behavior of the system, reducing the number of degrees of freedom (DOFs). The idea is to approximate the system transfer function accurately enough for a particular frequency range. Some of the general characteristics of these MOR methods are:

\footnotetext{
${ }^{1}$ There are other methods available, such as boundary element method (BEM).
} 
a. They are local (the model is reduced for a particular frequency range).

b. There is not a priori error bound.

c. They are very efficient computationally -managing easily models of order $\mathcal{O}\left(10^{6}\right)$ -

d. They can preserve some model features such as stability, passivity and RLC realizability of electrical circuits (resistors, inductors and capacitors). The characteristics preserved depend on the model to be reduced and the method chosen for model reduction.

One of the first methods that appeared involving the use of Krylov subspace was the asymptotic waveform evaluation (AWE) in 1990, followed by Padé via Lanczos (PVL) in 1995, PRIMA (passive reduced-order interconnect macromodeling algorithm) in 1998 and SyPVL (1996). Pieter Heres presents a thorough analysis of different Krylov subspace methods in [28].

\section{Systems and control}

This field focuses on the analysis of dynamical systems. The models try to represent very accurately the input-output behavior; therefore, the associated reduced models have the same goal. The models used are usually not as big as those used in numerical mathematics, but since the analysis of dynamical systems is ordinarily done to design a feedback controller, the models have to be solved in real time. Thus, the necessity of reduced order models.

Balance truncation realization (BTR) method is the most popular reduction approach for dynamical systems problems (an alternative to BTR is the optimal Hankel norm approximation). Some of its characteristics are:

a. It is global, aimed at the whole frequency range.

b. It provides an a priori error bound.

c. The method requires solving the Lyapunov equations, which makes it not efficient computationally. In practice, it limits the applicability of the method to models of order up to $\mathcal{O}\left(10^{3}\right)$.

d. It preserves the stability of the system.

Altough Besselink et al [18] is a very complete reference, they still leave out some reduction methods in their paper. In particular, all the techniques described in their study are "modelbased reduction techniques for linear time-invariant dynamical systems". A priori, none of the methods abovementioned would work for spacecraft thermal model reduction, given the strong nonlinear behavior caused by the thermal radiation terms. Nonetheless, they are discussed here because they have already been considered by some authors for spacecraft thermal models reduction purposes. In this regard, there are two methods that address precisely the nonlinear problems: trajectory piecewise linear (TPWL) and proper orthogonal decomposition (POD).

\section{Trajectory piecewise linear approach}

According to Rewienski and White in [29], the idea in the TPLW approach is to simulate the nonlinear system for a "training" input, obtaining a trajectory based on that input. Many linearized models are then generated around different points in the trajectory. The linearized models are assembled using state-dependent weights. The TPWL reduced model can be used to solve the nonlinear dynamic model. If the input signal originates a trajectory far enough from 
the one used for building the TPWL reduced model, the results could be not accurate enough. This situation can be solved by generating more TPWL models along different trajectories, covering a wider input space. The method does not provide a priori error bound, but the error can be estimated as the linear models are generated. It is noted by Rewienski that the generation of the linear models does not necessarily imply solving the full nonlinear model. Instead, it can be used an approximation of the full nonlinear model. This can be relevant if solving the nonlinear original model is costly. This is one important difference between TPWL and POD MOR techniques. The TPWL approach has been applied by Wang, Qian et al in the problem of spacecraft thermal model reduction [30, 31], and briefly commented by Deiml et al in [32]; these references will be discussed later. It has also been applied in nonlinear heat transfer problems by Yang and Shen in [33]. More details about TPWL MOR can be found in [34].

\section{Proper orthogonal decomposition}

POD is also known as Karhunen-Loève decomposition, least-squares estimation, and principal component analysis (PCA). In contrast with all the MOR techniques explained before (modelbased), POD is a data-based MOR method (although POD is frequently used just as a data analysis tool). It has typically been used in CFD, controllers design, signal analysis and inverse problems in structural dynamics. The method does not make any assumption about the system dynamics, and only relies on the dataset generated in an experiment or by analysis. The goal is to find an orthogonal reduced basis of the data, and as it turns out, POD produces an optimal basis in the sense of least-squares error of the data reconstruction (hence the name of leastsquares estimation). This data matrix can be constructed by using a dataset from an experiment -see for instance [35]- or assembling simulation data (by the so-called snapshots method introduced by Sirovich in [36]; every snapshot containing the values of the state variables for each time step, solved with the full-scale system). The matrix is then factorized by single value decomposition (SVD), and the obtained POD modes are truncated to some dimension lower than the original system. This reduced basis can be used to project the original system in this new subspace, obtaining a reduced order model. It is important to note that even though the POD modes are an optimal representation of the data, it does not imply their optimality for the reduced model generation. The reader can find more information regarding general POD applications and theory in [37], whereas POD application for spacecraft thermal model reduction is discussed in [31] and [32] and will be reviewed later in section 1.3.

This review of MOR methods does not intend to be exhaustive; there are many techniques precluded from the analysis. As mentioned before, good summaries can be found in [19, 20, 28], and also in $[38,39]$.

\subsection{Spacecraft thermal model reduction}

The literature regarding the spacecraft thermal model reduction is unfortunately not as abundant as for general MOR (it is more abundant, however, for reduction of thermal models in electronics and building construction, which are essentially linear systems, see for instance [40]). Below these lines, the "classical" or traditional methodology for spacecraft thermal model reduction is described, followed by the review of the most interesting references, generally ordered by publication date or by analogy order in few cases. 
In the spacecraft thermal modeling domain, the classical approach to deal with this situation is to set up a reduced geometrical mathematical model (RGMM). The reduced thermal mathematical model (RTMM) is built on a user-defined basis. This means the modeler builds the RGMM and, according to its definition, the nodes from the detailed thermal mathematical model (DTMM) are condensed into RTMM nodes. Then, the general practice is to get two snapshots of the DTMM, generally the hot and cold operational worst cases, which use to be the sizing ones, and, by tuning the conductive couplings and the heat loads, try to match the results from the RTMM and the DTMM. This process is time consuming, and tends to be error-prone. The main advantages of the classical approach are that the generated reduced model keeps the topological properties of a thermal mathematical model (symmetric conductive and radiative couplings matrices, with non-negative elements), no artificial couplings are created (although this totally depends on the engineer) and the RGMM can be integrated into another thermal model.

Jouffroy et al make in [41] a good description of the classical approach for TMM reduction. In what respect the automatization of the procedure indicated above, they present a thermal model reduction method for telecommunications satellite walls. The DTMM is set up with Airbus internally developed software GENASSIST, which produces high level of detail, and consequently a high number of nodes. The method they introduce is based on LPM models, and consists in mathematically generating an equivalent conduction matrix, inspired by the Guyan static condensation [22]. Nodes are divided in two types: control nodes (kept "as they are") and condensable nodes (to be condensed), following a static condensation reduction scheme (somehow already proposed by Bushard in [42], but not dealing with radiation terms). The "goal" temperature of a reduced model node is defined as the surface-weighted average of the nodes of the detailed model belonging to that reduced node. On the other hand, the radiative fluxes are computed following the assumption that the radiative heat flux of a detailed model node is a surface-weight fraction of the total corresponding to the reduced node to which the detailed node belongs. The errors in this reduction method-defined as differences between the surface-weighted average reduced model condensed nodes temperatures ("goal" temperatures) and those calculated by solving the reduced model itself- come from the radiative heat fluxes. The reduction is exact for pure conductive models. The main drawback is that the reduced conduction matrix can present couplings between reduced nodes that have no physical contact. In addition, some of the reduced couplings can be negative. DTMM nodes to be condensed into RTMM nodes seem to be defined by the user, which limits the automatization of the method.

Molina and Clemente follow in $[43,44]$ a similar approach. However, they introduce a very interesting concept: the energy balance between detailed and reduced nodes, which yields an equivalent reduced conductance: a sort of reference value for comparing the heat fluxes between reduced nodes and their equivalents in the detailed model.

In line with Jouffroy and Molina in [41, 43, 44], Bernard et al present in [23-27] a software called Thermal Model Reduction Tool (TMRT), which is distributed under license by Thales Alenia Space. The mathematical development of the method given in [24] makes easier to follow the different steps involved in the model reduction (compared to the papers discussed earlier). A new category of nodes, called "suppressed" -which are neither condensed nor kept in the RTMM- is defined by the authors. These nodes are limited to those coupled to their neighbors only by conduction. 
Kim and Kim use in [45] the same approach, giving a very simple numerical example, which helps to understand part of the algebra involved. Kim and Kim use the software COMSTAP (developed by the Korean Space Research Institute) to generate the thermal mathematical model. In this paper, the authors define the reduced nodes by grouping nodes that are within a temperature difference range, with the help of the above-mentioned software (Fernández-Rico et al do something similar in [46], which is the basis for this thesis dissertation). Kim and Kim establish the relation between the level of isothermality of the condensed nodes in the definition phase and the temperatures obtained by solving the RTMM.

In [47], Krishnamoorthy and Chowdhury also use static condensation for compact thermal modelling of electronic components. This paper is unrelated to spacecraft thermal modelling, and they do not include radiation terms, but the interesting thing is that they suggest the representation of the thermal network as an undirected graph.

Following a completely different methodology, Noor et al propose in [48] to reformulate the nonlinear steady state thermal problem in terms of a reduced basis, calculated by making use of perturbation techniques and the Bubnov-Galerkin method (the math involved is very complex). The analysis uses the finite element method; the aim is to generate a lower order system for steady state analysis, but not to generate a stand-alone model to be included in a higher hierarchy model.

Gorlani and Rossi present in $[49,50]$ a stochastic optimization for thermal model reduction. The method is divided in two steps. The first step deals with the geometrical mathematical model (GMM), and aims to define the reduction scheme, that is, the correspondence between DTMM and RTMM nodes. This seems to be done manually by the engineer, and takes (usually) a single iteration. The second step takes the reduced model conductive couplings as variables (the existing reduced conductors that have been already stablished), and performs a full factorial analysis with stochastic optimization to determine the reduced conductors that best fit the goal temperatures. The stochastic optimization is carried out with PANAMA, a software tool developed by the authors. The reduced model ends up with positive reduced conductive couplings, no artificial conductors, with a reduction ratio of about $80 \%$. The reduction errors are defined, as before, by comparing the defined and calculated reduced model nodes temperatures, and by computing the root sum square of the temperature differences indicated before. This reduction approach has some similarities with thermal model correlation methods, which will be referred to further on.

The application of POD and TPWL to spacecraft thermal model reduction is studied by Wang, Qian et al in $[30,31]$. The goal of the studied approaches is not to generate a new model to be integrated within other models, but to obtain results under new boundary conditions and environmental fluxes. An interesting aspect to be mentioned is that the paper discusses in its introduction the data-driven heuristic models, posing them as not suitable for dynamic behavior prediction. To the best of our knowledge, these heuristic models have not been used among European space thermal community. TPWL shows an excellent performance with the case studies. As mentioned in section 1.2, the usability of the reduced model can be limited if the new inputs intended to be simulated differ too much from those used for the reduced model generation. POD, as discussed before, is a very popular nonlinear MOR method. It shows also good performance for the spacecraft thermal reduction. POD presents an important limitation: the necessity of evaluating the nonlinear terms in the detailed model, which limits the 
computational efficiency. Qian et al propose the use of discrete empirical interpolation method (DEIM, see [51]) to mitigate this calculation burden. A couple of examples are given in the paper, showing a good performance of both methods.

Deiml et al make in [32] a clear distinction between GMM and TMM in the process of reducing a spacecraft thermal model. The model has the environment -including deep space-, the boundaries (which are the nodes/geometries that have couplings with the environment) and internal nodes/geometries (which are only connected to the model boundaries). The first step is to reduce the GMM externally. Model geometry is simplified, trying to match heat flows between environment and the boundaries, by varying the thermo-optical properties -which will have an influence in the radiative exchange factors, REFs. To reduce the pure geometry, polygon reduction techniques are explored and several methods are tested by the authors in order to adjust the thermo-optical properties:

- Sequential Quadratic Programming (SPQ) algorithm [52].

- Global optimization derivative free algorithm [53].

The results obtained with the polygon reduction techniques are not very satisfactory, so the manual reduction is considered as the most effective way. More research is necessary in this area, since most of the references do not even mention the problem of reducing the GMM, so in this respect Deiml is very innovative. Regarding the adjustment of thermo-optical properties for heat fluxes matching, the SPQ algorithm does not work as expected, but the Global optimization derivative free algorithm seems to fulfill the requirements and yield good results.

The second step in the reduction process is the simplification of the TMM concerning heat fluxes between environment and boundaries. Again, this can be done by modifying the thermooptical properties. Unfortunately, no information is given by the authors about what happens to conductive fluxes.

Third step is to reduce the internal nodes. For that, linear and nonlinear MOR methods are investigated by the authors. The following linear reduction methods are suggested in the paper: Guyan, Truncated Balance Realization and Krylov subspace methods. Their results are not listed in the case studies shown in the paper, as they are not considered useful. Regarding nonlinear methods, POD is tested but it is not considered useful for the reduction, due to the necessity of calculating the nonlinear terms (also mentioned in [31]). TPWL is not applicable for the goal stated in the paper, which is the further integration of the reduced model in other models -TPWL only allows to solve the system to get results. Balancing for nonlinear systems [54] is very difficult to implement according to the authors. Apart from the methods explained above, three model reduction techniques are developed in the article:

- Empirical model reduction technique (no insight of this method is provided in the paper text).

- Matrix-based reduction method (also no specific details are given; it is described as "an exact reduction of the thermal model along a reference trajectory, typically a superposition of hot and cold cases").

- Summation method is chosen as the best one among those tested. The performance and solution runtime are considered adequate, but the description is limited to the name, so it is difficult to judge the adequacy for different models and circumstances. 
The first two methods are not considered useful by Deiml et al, as the lack of performance and the drawbacks are excessive.

Hengeveld and Biskner present in $[55,56]$ a novelty approach different from nodal reduction methods, since it relies in the data generated by solving the detailed thermal mathematical model. The approach does not aim to generate a reduced model as such (in the sense that can be implemented in a higher hierarchy model), but to obtain results with alternative configurations (different inputs for the detailed model). The development of the reduced model is based on sampling the input space and fitting the data obtained by running the detailed model in the sampled points. Latin Hypercube Sampling (LHS) pursues the sampling of the design space, defined as the combinations of values of different input parameters -for instance, boundary temperatures and physical properties- in the model definition. Once the design space has been sampled with LHS, the detailed model is run for each point in that design space (training input), producing training results for the data fitting. The outputs are then modeled as an overall mean plus a Gaussian process, thus mapping the inputs to outputs. The proposed method is judged very interesting and versatile for sensitivity analysis and finding possible design optimums, but it is not suitable for producing reduced order models that can be combined with other models.

A model reduction for FEM thermal models is proposed by Jacques et al in [57]. FEM is not as popular as the lumped parameter method within the spacecraft thermal analysis community. One of the reasons (among many others, see [58]), is the computational cost of calculating the REFs by means of the Monte Carlo ray tracing method. This difference in computational cost is because FEM model meshes are usually much more dense than LPM ones. Nevertheless, the use of FEM models is particularly interesting when using a mesh coming from a structural model. Jacques proposes the use of mesh clustering and super nodes to alleviate the problem of the calculation runtimes. For the calculation of REFs, the finite element mesh is clustered starting from the seed locations defined by the engineer. The reduction of the conductive model to super nodes relies on the zones already created by the mesh clustering. The determination of the reduced conductive couplings matrix is done by expanding the linear matrix system with the super nodes temperatures, and assuming that the loads are uniform within a super node. The authors also define a procedure that avoids the necessity of inverting the extended coefficients matrix for finding the reduced model conductive couplings matrix. This MOR approach seems to be in general very efficient and complete, but it presents some limitations for our purposes: the assumption that the loads are uniform within the super nodes, and the necessity of userdefined seeds for mesh clustering, which in turn define the super nodes. For the adaptation of a structural FEM mesh for thermal analysis, the method performance seems outstanding.

From the review of all the references above, one can notice that in general, the GMM reduction is left unaddressed. In addition, the reduction scheme (how the detailed model nodes are grouped and condensed into reduced nodes) is often defined by the user, rather than determined automatically by an algorithm.

\subsection{Objectives and content structure}

Without demeaning all the techniques and methods described in the previous section, in our opinion there is some space for improving the spacecraft thermal model reduction. Our idea is to use the physical insight of the thermal models to reduce their complexity. The aim shall be to reproduce the input-output behavior (basically the heat fluxes through the boundaries), as well 
as the inner behavior, that is, the system temperatures, while keeping the structure of a LPM thermal model, so that it can be further integrated with other thermal models (thus, not just a black box that matches the DTMM results). Aiming to guarantee that the RTMM resulting temperatures are really representative of the behavior predicted by the DTMM, it is necessary to preserve the hot and cold spots during the reduction process. For this reason, the condensation of nodes here proposed will be based on the identification of quasi-isothermal zones. The nodes part of these isothermal zones will be potential candidates to be condensed, provided that they are in physical contact.

Summarizing, the main goals and characteristics of the quasi-autonomous thermal reduction process presented here are:

a. Reduce the thermal model taking into account the different quasi-isothermal zones. By grouping similar-temperature nodes, it is possible to reduce the error produced by reducing the matrices. Furthermore, it can be supposed that parts that are strongly connected -larger conductive couplings- will be more isothermal than other parts that are weakly connected.

b. The model reduction should be done in a quasi-automatic way. This means, the only parameters that need to be defined are the maximum allowable temperature and heat fluxes differences, as well as the desired reduction ratio.

c. The reduction process will depend on the temperatures and boundary conditions. Having more scenarios (e.g. hot and cold operational cases) will constrain the problem more.

d. In engineering applications, it is essential to preserve the physical interpretation of the model elements, as well as the physical characteristics, to be able to take design decisions changing physically achievable parameters. This implies having positive thermal couplings, to preserve the boundary nodes, the heat loads, the symmetry in the coupling matrices, and the conductive physical paths. We can define this as structurepreserving reduction.

This dissertation is divided in six chapters. The first chapter (above these lines) corresponds to the introduction and the literature review. The second chapter provides a brief theoretical introduction to the reduction process. Chapter 3 describes the main quantities and parameters in a thermal mathematical model. Chapter 4 gives a detailed description of the reduction process. In chapter 5, the reduction process is tested on two models. Chapter 6 summarizes the conclusions and some ideas for future developments. 


\section{THEORETICAL BACKGROUND}

Before entering into the full description of the reduction method, let us start with a brief theoretical introduction to describe the condensation philosophy. First, a 4-node purely conductive model is analyzed, and then the conclusions found are applied on a general basis.

\subsection{Reduction analysis of 4-node model}

The couplings among the four nodes that form the model are shown in Fig. 2-1. It simulates a body made of two parts (nodes 1 and 2 on one side, and nodes 3 and 4 on the other) that internally are strongly connected, but are weakly connected to each other. There is a fifth node labeled as $B$ that represents a boundary condition in temperature. It is included to avoid the singularity of the system.

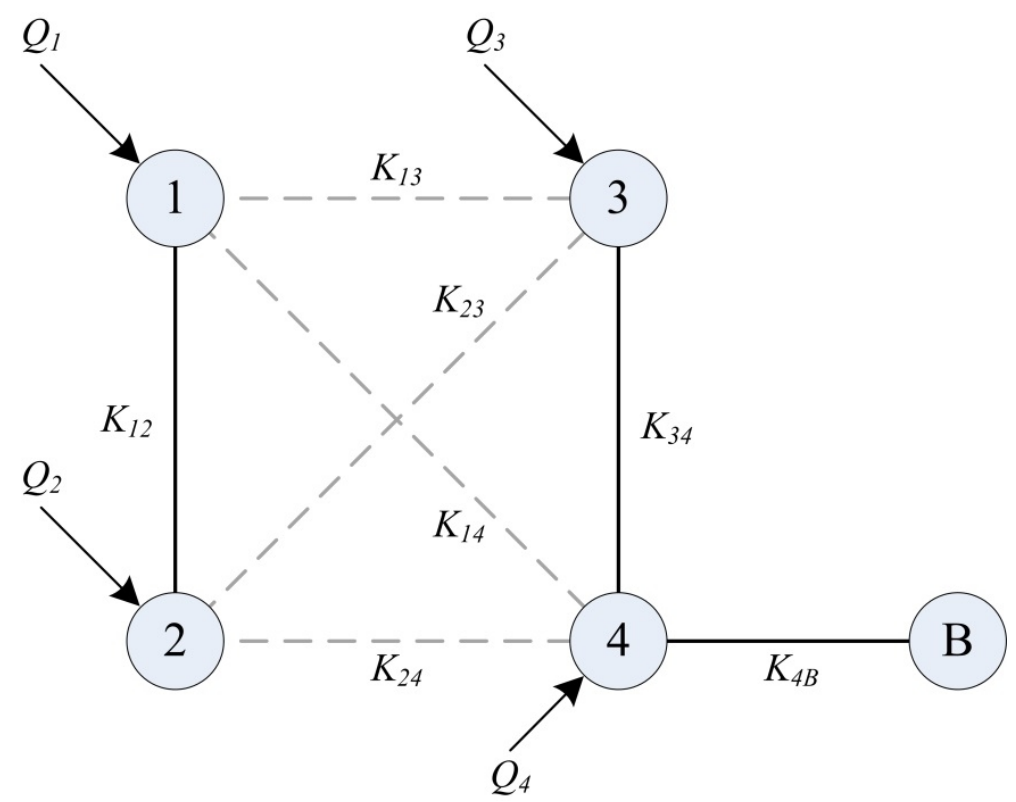

Fig. 2-1. 4-node model schema.

The couplings of the system in this example are such that

$$
K_{13} \sim K_{14} \sim K_{24} \sim K_{23} \ll K_{12} \sim K_{34}
$$

The sign convention is $Q_{i}>0$ when heat enters the node. We can write the energy balance equations, one per node:

$$
\left\{\begin{array}{l}
K_{12}\left(T_{2}-T_{1}\right)+K_{13}\left(T_{3}-T_{1}\right)+K_{14}\left(T_{4}-T_{1}\right)+Q_{1}=0 \\
K_{12}\left(T_{1}-T_{2}\right)+K_{23}\left(T_{3}-T_{2}\right)+K_{24}\left(T_{4}-T_{2}\right)+Q_{1}=0 \\
K_{13}\left(T_{1}-T_{3}\right)+K_{23}\left(T_{2}-T_{3}\right)+K_{34}\left(T_{4}-T_{3}\right)+Q_{3}=0 \\
K_{14}\left(T_{1}-T_{4}\right)+K_{24}\left(T_{2}-T_{4}\right)+K_{34}\left(T_{3}-T_{4}\right)+K_{4 B}\left(T_{B}-T_{4}\right)+Q_{4}=0
\end{array} .\right.
$$


In matrix form:

$$
\mathbf{K} \mathbf{T}=\mathbf{Q}
$$

where

$$
\begin{gathered}
\mathbf{K =} \\
\left(\begin{array}{cccc}
-\left(K_{12}+K_{13}+K_{14}\right) & K_{12} & K_{13} & K_{14} \\
K_{12} & -\left(K_{12}+K_{23}+K_{24}\right) & K_{23} & K_{24} \\
K_{13} & K_{23} & -\left(K_{13}+K_{23}+K_{34}\right) & K_{34} \\
K_{14} & K_{24} & K_{34} & -\left(K_{14}+K_{24}+K_{34}+K_{4 B}\right)
\end{array}\right) \\
\mathbf{T}=\left(\begin{array}{c}
T_{1} \\
T_{2} \\
T_{3} \\
T_{4}
\end{array}\right), \mathbf{Q}=\left(\begin{array}{c}
-Q_{1} \\
-Q_{2} \\
-Q_{3} \\
-Q_{4}-K_{4 B} T_{B}
\end{array}\right) .
\end{gathered}
$$

Summing up the four equations in (3), we get

$$
K_{4 B}\left(T_{B}-T_{4}\right)=-Q_{1}-Q_{2}-Q_{3}-Q_{4} \rightarrow T_{4}=\frac{\sum_{i=1}^{n_{d}} Q_{i}}{K_{4 B}}+T_{B} .
$$

Expression (7) allows us to fix a temperature level for the system. Based on the relations in (2), we can associate the nodes 1 and 2 on one side, and nodes 3 and 4 on the other, forming two reduced nodes called $N_{c_{1}}$ and $N_{c_{2}}$ respectively. Let us call the temperatures of the reduced nodes as $T_{1}^{R}$ and $T_{2}^{R}$. For each node $i$ of the detailed model:

$$
T_{i}=T_{j}^{R}+\tau_{i}, \quad i=1, \ldots, n_{d}, \quad j=1, n_{c} ;
$$

$\tau_{i}$ represents the difference between the temperature of node $i$ and the temperature of its associated reduced node $N_{c_{j}}$. We can reorganize the system in (5) and (6), using (8), by condensed nodes:

$$
\left(\mathbf{K}_{0}+\mathbf{K}_{1}\right)\left(\mathbf{T}^{\mathrm{R}}+\boldsymbol{\tau}\right)=\mathbf{Q}
$$


where $\mathbf{K}=\mathbf{K}_{0}+\mathbf{K}_{1}$, which are defined as follows

$$
\begin{gathered}
\mathbf{K}_{0}=\left(\begin{array}{cccc}
-K_{12} & K_{12} & 0 & 0 \\
K_{12} & -K_{12} & 0 & 0 \\
0 & 0 & -K_{34} & K_{34} \\
0 & 0 & K_{34} & -K_{34}
\end{array}\right), \\
\mathbf{K}_{1}=\left(\begin{array}{cccc}
-\left(K_{13}+K_{14}\right) & 0 & K_{13} & K_{14} \\
0 & -\left(K_{23}+K_{24}\right) & K_{23} & K_{24} \\
K_{13} & K_{23} & -\left(K_{13}+K_{23}\right) & 0 \\
K_{14} & K_{24} & 0 & -\left(K_{14}+K_{24}+K_{4 B}\right)
\end{array}\right),
\end{gathered}
$$

the terms in eq. (9) can be expanded, yielding

$$
\overbrace{\mathbf{K}_{0} \mathbf{T}^{\mathrm{R}}}^{\mathrm{a}}+\overbrace{\mathbf{K}_{1} \mathbf{T}^{\mathrm{R}}}^{\mathrm{b}}+\overbrace{\mathbf{K}_{0} \boldsymbol{\tau}+\mathbf{K}_{1} \boldsymbol{\tau}}^{\mathrm{c}}=\mathbf{Q} .
$$

Let us analyze the different elements in (12):

a. $\quad \mathbf{K}_{0} \mathbf{T}^{\mathrm{R}}=0$ : This represents the heat fluxes within the nodes belonging to the same reduced node, assuming that their temperatures are the same as the reduced node one. Obviously, these heat fluxes will be zero. For instance, for the first row:

$$
-K_{12} T_{c_{1}}+K_{12} T_{c_{1}}=0 \text {. }
$$

b. $\quad \mathbf{K}_{1} \mathbf{T}^{\mathrm{R}}=\mathbf{Q}$ : gives the equations to calculate the reduced nodes temperatures. For the first two rows:

$$
\left\{\begin{array}{l}
-\left(K_{13}+K_{14}\right) T_{c_{1}}+K_{13} T_{c_{2}}+K_{14} T_{c_{2}}=-Q_{1} \\
-\left(K_{23}+K_{24}\right) T_{c_{1}}+K_{23} T_{c_{2}}+K_{24} T_{c_{2}}=-Q_{2}
\end{array} .\right.
$$

Grouping by the difference $\left(T_{c_{2}}-T_{c_{1}}\right)$ :

$$
\left\{\begin{array}{l}
\left(K_{13}+K_{14}\right)\left(T_{c_{2}}-T_{c_{1}}\right)=-Q_{1} \\
\left(K_{23}+K_{24}\right)\left(T_{c_{2}}-T_{c_{1}}\right)=-Q_{2}
\end{array} .\right.
$$

Summing up both equations, we get

$$
\left(K_{13}+K_{14}+K_{23}+K_{24}\right)\left(T_{c_{2}}-T_{c_{1}}\right)=-\left(Q_{1}+Q_{2}\right) .
$$

Analogously, for rows 3 and 4 in $\mathbf{K}_{1} \mathbf{T}^{\mathrm{R}}=\mathbf{Q}$ :

$$
\left(K_{13}+K_{14}+K_{23}+K_{24}\right)\left(T_{c_{1}}-T_{c_{2}}\right)-K_{4 B} T_{c_{2}}=-\left(Q_{3}+Q_{4}+K_{4 B} T_{B}\right) .
$$


Combining (16) and (17):

$$
K_{4 B} T_{c_{2}}=Q_{1}+Q_{2}+Q_{3}+Q_{4}+K_{4 B} T_{B} \rightarrow T_{c_{2}}=\frac{\sum_{i=1}^{n_{d}} Q_{i}}{K_{4 B}}+T_{B} .
$$

Given the structure of the model (unique boundary condition solely connected to a single node), eq. (18) coincides with eq. (7). We can use the expression for $T_{c_{2}}$ given by (18) in equation (16), to get $T_{c_{1}}$ :

$$
T_{c_{1}}=\frac{Q_{1}+Q_{2}}{K_{13}+K_{14}+K_{23}+K_{24}}+T_{c_{2}} .
$$

The reduced model, with the aggregated loads and couplings, is shown in Fig. 2-2.

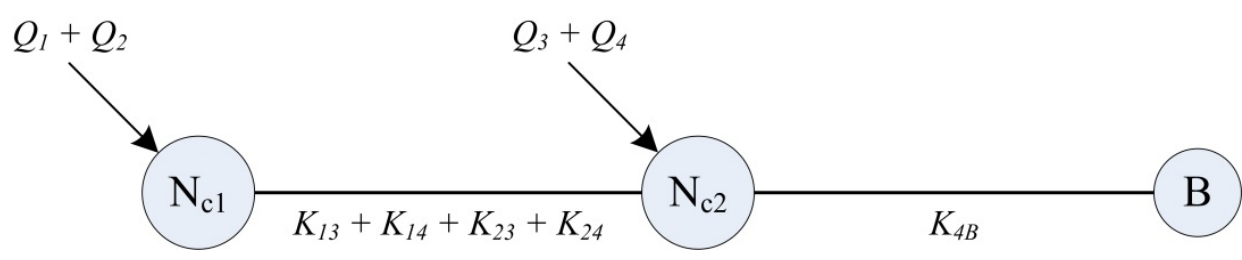

Fig. 2-2. Condensed 4-node model schema.

c. $\left(\mathbf{K}_{0}+\mathbf{K}_{1}\right) \boldsymbol{\tau}=0$ describes the heat fluxes among the nodes in the detailed model, as a consequence of the deviation between the original model temperatures and its associated reduced nodes ones. Having found the reduced thermal model node temperatures with (12)-b, we can calculate the deviations between $T_{i}$ and $T_{j}^{R}$. Of course, solving the system (4) allows us to get the detailed model temperatures $\mathbf{T}$, and then use together with $\mathbf{T}^{\mathbf{R}}$ in (8) to obtain $\boldsymbol{\tau}$.

\subsection{General approach}

Consider a thermal model consisting in $n$ thermal nodes described by its coupling matrix $\mathbf{K}$. Let us have matrix $\mathbf{K}$ already sorted by the reduced nodes, so as shown in (9) $\mathbf{K}=\mathbf{K}_{0}+\mathbf{K}_{1}$ :

a. $\mathbf{K}_{\mathbf{0}}$ is a block diagonal matrix; each block groups the nodes belonging to the same reduced thermal model node. In principle, $\mathbf{K}_{\mathbf{0}}$ has the higher order couplings $K_{i j}$.

b. $\quad \mathbf{K}_{1}$ is a matrix with blocks out of the diagonal; each block groups the couplings $K_{i j}$ (presumably lower order) between nodes belonging to different reduced thermal model nodes.

Regarding the temperatures, as per (8) $\mathbf{T}=\mathbf{T}^{\mathrm{R}}+\boldsymbol{\tau}$ :

a. $\quad \mathbf{T}^{\mathbf{R}}$ is a vector with the reduced nodes temperatures. For matrix operations, $\mathbf{T}^{\mathbf{R}}$ would have $n_{c}$ different components (one per reduced thermal model node), each of them repeated as many times as the amount of detailed model nodes condensed in each reduced node.

b. $\tau$ represents the temperature difference between the detailed mode node and its associated reduced node. 
The system is defined by the eq. (4), which can be expanded to get the expression (12), written again below for clarity:

$$
\mathbf{K} \mathbf{T}=\overbrace{\mathbf{K}_{0} \mathbf{T}^{\mathrm{R}}}^{=0}+\overbrace{\mathbf{K}_{1} \mathbf{T}^{\mathrm{R}}}^{\mathrm{b}}+\overbrace{\left(\mathbf{K}_{0}+\mathbf{K}_{1}\right) \boldsymbol{\tau}}^{\mathrm{c}}=\mathbf{Q} .
$$

The reduced thermal model temperatures can be obtained with $\mathbf{K}_{1} \mathbf{T}^{\mathrm{R}}=\mathbf{Q}$, combining rows and columns by condensed nodes (also compacting vectors $\mathbf{T}^{\mathbf{R}}$ and $\mathbf{Q}$ ), to get a system of dimension $n_{c}$. The equation (4) can be solved to get the detailed model temperatures

$$
\mathbf{T}=\mathbf{K}^{-1} \mathbf{Q} \text {. }
$$

This allows assessing the temperature differences $\tau_{i}$. The better the reduction the smaller should be $\tau_{i}$.

If the thermal problem involves also radiation, there will be another matrix called $\mathbf{R}$, with the radiative couplings between the nodes of the detailed model. Since the radiative heat exchange goes with the difference of the fourth power of the temperatures, this makes the problem nonlinear. One can try to linearize the system around the solution point, and incorporate the linearized terms in $\mathbf{K}$, getting a new matrix called $\mathbf{K}^{*}$. In principle, the whole approach explained above these lines is valid, regardless of the existence of radiation or not.

The quality of the reduction process is based in the way the nodes of the detailed model are grouped together. We need a systematic approach to select the nodes belonging to the same reduced thermal model nodes, reducing the model; that is, determining the thermal couplings between reduced thermal model nodes, keeping the temperature differences $\tau_{i}$ as small as possible or below a previously defined threshold, and matching the heat exchanges between the model and its boundaries. The following chapters are devoted to develop and test the reduction algorithm. 



\section{THERMAL MODEL}

The lumped parameter method thermal mathematical model is described by Eq. (22), one energy balance equation per node [59]:

$$
C_{i} \frac{d T_{i}}{d t}=\sum_{j=1}^{n_{d}}\left[K_{i j}\left(T_{j}-T_{i}\right)+R_{i j}\left(T_{j}^{4}-T_{i}^{4}\right)\right]+Q_{i}, \quad i=1, \ldots, n_{d}, \quad i \quad j \quad,
$$

where $C_{i}$ is the thermal capacitance of node $i, K_{i j}$ and $R_{i j}$ the conductive and radiative couplings between nodes $i$ and $j$, respectively, and $Q_{i}$ the heat load per unit of time on node $i$. $C_{i}$ includes the nodal volume, specific heat and density. $K_{i j}$ includes the effect of nodal areas, distances, thermal conductivities and thermal contact conductances involved in the calculation. $R_{i j}$ includes the Stefan-Boltzmann constant, $\sigma . R_{i j}$ includes also the different parameters used for its determination: view factors, thermo-optical properties and areas. As indicated before, the sign convention is $Q_{i}>0$ when heat enters the node. The coefficients of these equations are usually generated with a thermal analysis software package, but they can be partially or totally produced by other means. For the development and execution of the reduction algorithm, the reference software is ESATAN-TMS, as one of the standard tools for space thermal analysis in Europe. In particular, all the magnitudes contained in the ESATAN thermal model data ${ }^{2}$ (TMD) output file will be considered known, and are the base for the numerical method developed in this work. Thus, all the variables that appear in eq. (22) are considered as available inputs. These are $\mathbf{K}$ (conductive coupling matrix), $\mathbf{R}$ (radiative coupling matrix), $\mathbf{T}$ (temperature vector), $\mathbf{C}$ (node thermal capacity vector) and $\mathbf{Q}$ (node heat load vector). Also the node spatial coordinate matrix, $\mathbf{X}$, is considered to be known.

In general, it could be also considered that the material properties (thermal conductivity, specific heat and density) of the different parts of the thermal model are known. However, while these properties allow the calculation of some of the matrices and vectors indicated in equation (22), they are not explicitly output by ESATAN in the TMD file.

We can delimit a little bit more the available inputs. As it will be shown in section 4.2.1, the node associated geometry thickness could be very useful for the model reduction. However, this information is not available in the ESATAN TMD output file, and therefore it is not used in the reduction method. In addition, the nodal area could be helpful, and it is output in the TMD file, but the way in which this is output is ambiguous ${ }^{3}$.

\footnotetext{
${ }^{2}$ TMD is a thermal data file formatted in Hierarchical Data Format (HDF) Version 5.

${ }^{3}$ When radiative active geometry, a shell defined in the GMM with the same node number on both sides will account twice for the face area, whereas if the shell has different node numbers, the output surface indeed corresponds to the real face area. In general, it is impossible to distinguish between both situations based on what is output in the TMD file.
} 



\section{REDUCTION PROCESS}

\subsection{Scope}

Reducing pure resistive electric circuits networks -with the same behavior as linear thermal models, that is, those exclusively made of conductive couplings- has been addressed in some references $[60,61]$ and can be done to get exact results in terms of heat fluxes through the boundaries. However, reducing nonlinear models (in principle, those that include radiative terms) can lead to excessive temperature deviations between the detailed and the reduced models, and consequently produce excessive heat flux differences through the boundaries. Normally, nodes with similar temperatures -and that can therefore be considered as condensable- are internally conductively coupled. Radiation is usually not as important as conduction. Thus, for the reduction, groups of nodes physically well connected and with a similar temperature level are sought. In addition, it is considered here that two nodes can be condensed only if they are connected by a material medium, so that the physical meaning is preserved. That is why only the conductive couplings matrix $\mathbf{K}$ is taken into account to run the reduction algorithm. Once the model has been reduced, the radiative couplings matrix $\mathbf{R}$ is reduced using the same scheme and the problem can be linearized and solved.

A flowchart of the reduction process that is explained in the subsequent sections is shown in Fig. 4-1. Detailed view of the process can be found in Fig. 4-2. The goal of the reduction process is to find, in an autonomous way, the scheme that condenses the detailed thermal model nodes into reduced thermal model nodes. In particular, the aim is to transform the matrix system dimension from $n_{d}$ (number of nodes of the detailed model, including the boundary nodes) to $n_{c}$ (number of nodes of the reduced model, including the boundary nodes). The relation between $n_{d}, n_{c}$ and $n_{b}$ (number of boundary nodes, invariant in the reduction process) is here called reduction ratio $r_{r}$, which is a measure of the reduction degree. $r_{r}$ is defined as

$$
r_{r}=1-\frac{n_{c}-n_{b}}{n_{d}-n_{b}}
$$

$r_{r}$ ranges from 0 to $1 ; 0$ indicates that the reduced and the detailed models are the same, whereas 1 represents complete reduction, as only the boundary nodes remain in the reduced model ${ }^{4}$.

To transform the system, a matrix $\mathbf{A}$ is built. $\mathbf{A}$ indicates the connection between nodes, taking into account the conductive coupling $\mathbf{K}$ and the temperature difference between nodes $\boldsymbol{\Theta}$. Matrix $\mathbf{A}$ is treated as an adjacency matrix, on which the strongly ${ }^{5}$ connected components (SCC) algorithm [62-64] is used. The resulting reduction scheme (represented by restriction matrix $\mathbf{P}$ ) is applied to the thermal problem, reducing the dimension of matrices $\mathbf{K}^{\mathrm{R}}, \mathbf{R}^{\mathrm{R}}, \mathbf{Q}^{\mathrm{R}}$.

\footnotetext{
${ }^{4}$ In reality, as it will be shown in the chapter, this situation is not possible, since at least one diffusion node has to remain in the condensed model. Nevertheless, ideally $r_{r}=1$ represents the upper limit of the reduction ratio.

${ }^{5}$ The thermal network is represented as an undirected graph. For such type of graph, it makes no sense to use the word "strongly" to define the connected components, but still it has been chosen to keep it, as it is used in the generic algorithm used for finding the condensed nodes [64].
} 
Then this system is solved, and the calculated temperatures $\hat{\mathbf{T}}^{\mathrm{R}}$ and heat fluxes $\hat{\mathbf{H}}^{\mathrm{R}}$ are compared to those derived from the detailed thermal model, $\overline{\mathbf{T}}^{\mathrm{D}}$ and $\mathbf{H}^{\mathrm{D}}$ respectively. An iteration loop could be set up to find the threshold values to fulfill the requirements imposed on both temperature $\left(\delta_{\max }\right)$ and heat flux differences $\left(q_{\max }\right)$ between the reduced and the detailed TMMs. If the correlation requirements $\delta_{\max }$ and $q_{\max }$ are too strict, it might be impossible to achieved the required reduction ratio, $r_{r, m i n}$.

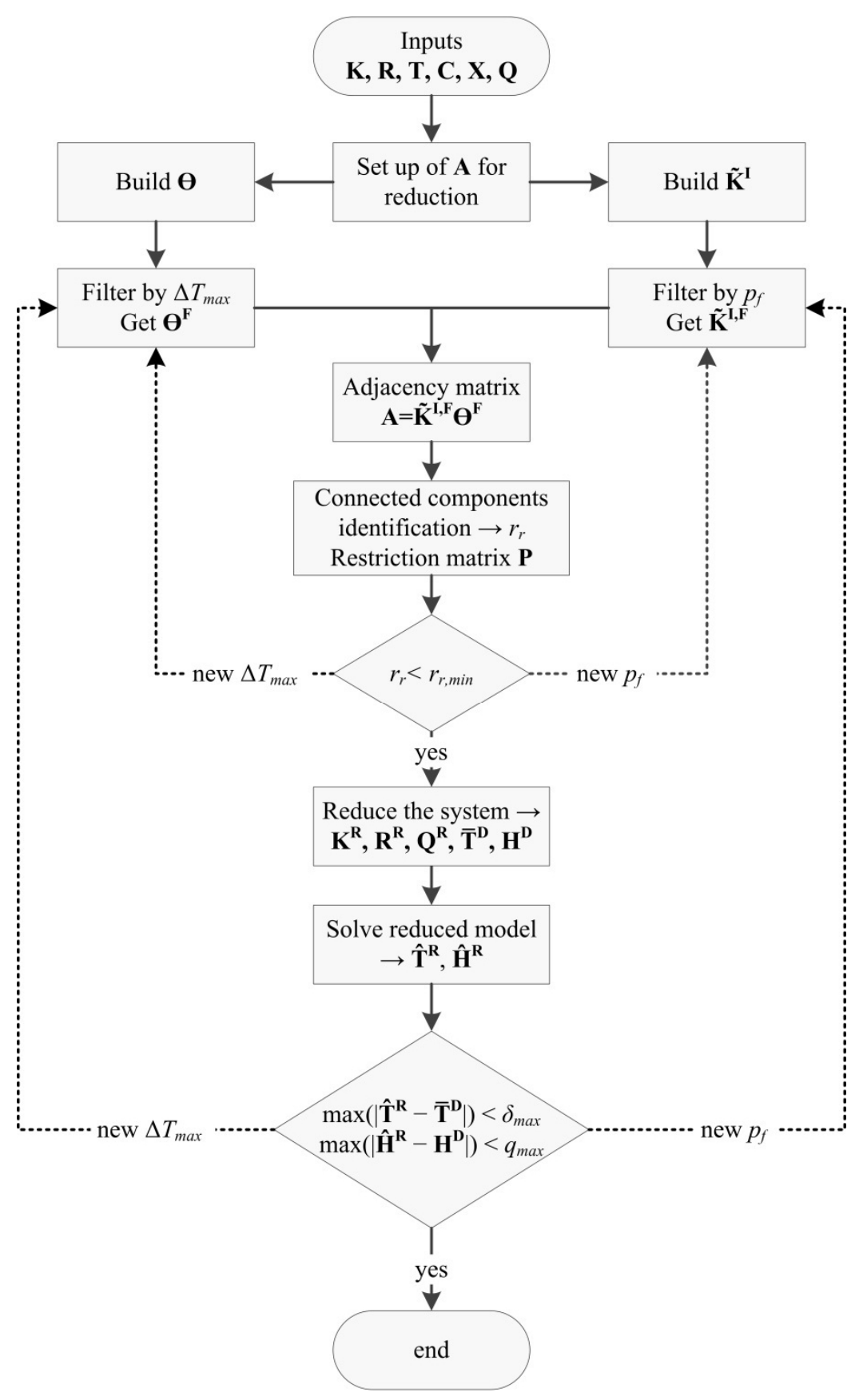

Fig. 4-1. Reduction process flowchart. 


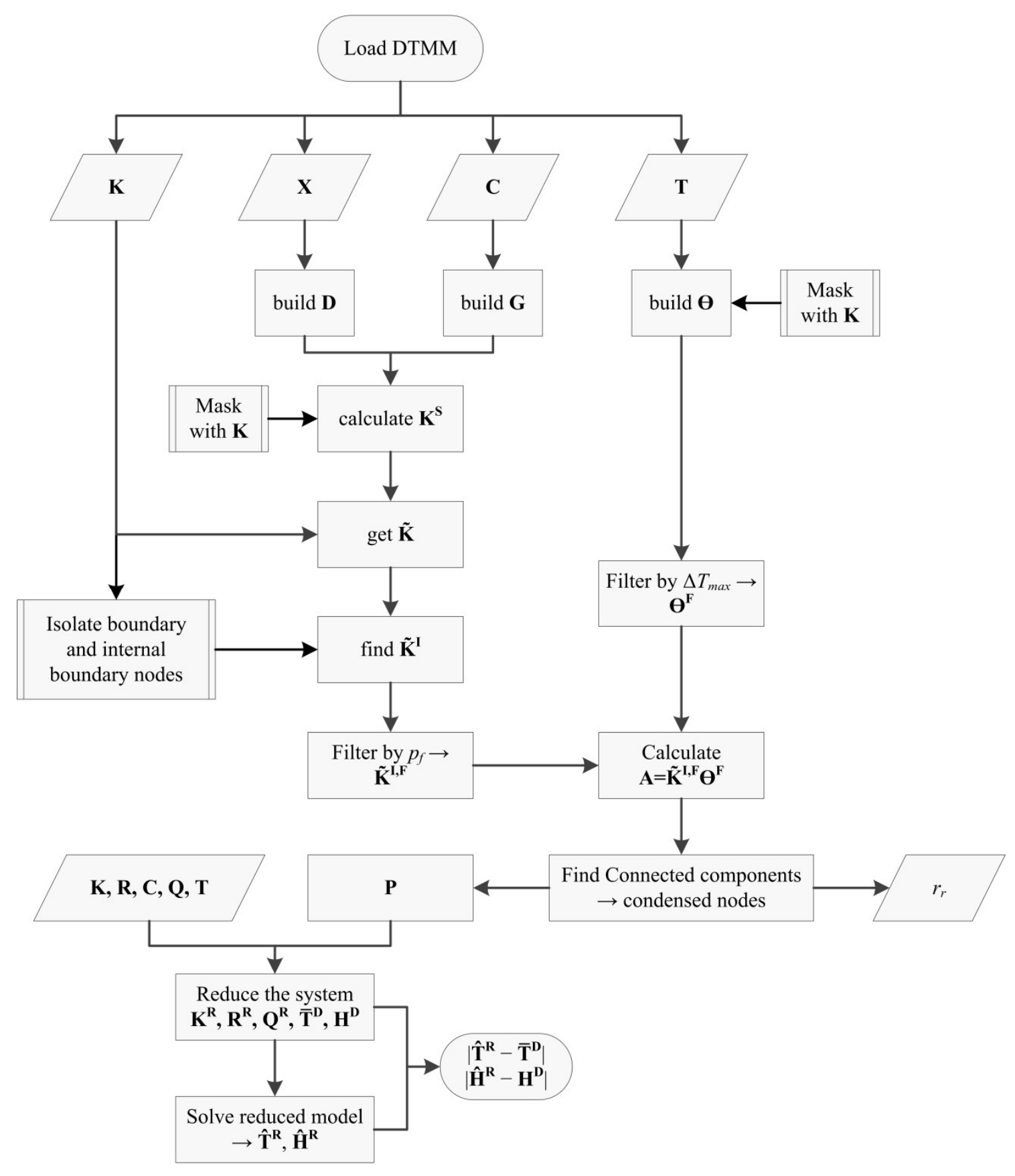

Fig. 4-2. Reduction process flowchart - detail.

All the steps described above have been programmed with Python 3.4 and the Python-based scientific ecosystem SciPy ${ }^{6}$. Compressed Storage by Rows ${ }^{7}$ (CSR) sparse matrices have been used in order to save memory and make the code more efficient.

${ }^{6}$ https://www.scipy.org/about.html 


\subsection{Processing of conductive coupling matrix $\mathbf{K}$}

The conductive couplings between the different nodes in a thermal model are calculated based on geometrical and physical properties, as an application of Fourier's law in a lumped parameter model [65-67]. Usually, the values generated in this way are within quite a wide range (eight orders of magnitude, approx. $10^{-5}$ to $10^{+3} \mathrm{~W} / \mathrm{K}$ ). One of the most important contributions of this study is to realize that to compare and classify the different values as "strong" or "weak" conductors, one has to take into account not only the values themselves, but also the geometrical and physical properties which have produced such values. It should be considered that, when constructing a thermal model, different spatial scales are frequently used for different parts of the geometrical model, and they largely influence the values of the couplings. For instance, anticipating that metallic parts, such as structural panels, will be quasi-isothermal, one can produce a coarser mesh. On the other hand, modelling printed circuit boards, where it could be important to distribute accurately the electric power sources, can lead to a finer mesh. This situation can produce thermal models with a great disparity of node sizes, and therefore of thermal coupling values. The diversity of bulk materials in the model construction, the simulation of thermal contacts at the bolted or glued interfaces, and other effects, greatly influence the inhomogeneity of the conductors values. If the nodes are going to be grouped based on the values of the conductors involved, it will be necessary to assess the relationship between the conductor actual value, and the conductor potential value -how big this conductor could be- based on the available magnitudes. To help making this comparison, a dimensionless conductive coupling matrix, $\tilde{\mathbf{K}}$, is built.

\subsubsection{Building the dimensionless coupling matrix $\tilde{\mathbf{K}}$}

To obtain the dimensionless conductive coupling matrix $\tilde{\mathbf{K}}$, characteristic values of $K_{i j}$ are needed to be used as a reference. These reference values are grouped inside the conductive coupling sizing matrix, $\mathbf{K}^{\mathrm{S}}$. As we want to identify which nodes should be condensed, we are interested in having orders of magnitude for the conductive couplings in $\mathbf{K}^{\mathrm{S}}$ based on the size of the contact and the materials involved. In LPM models, the thermal capacity is concentrated in the nodal center, and this capacity represents somehow the geometry. The geometry is taken into account also when calculating the conductive and radiative couplings. $\mathbf{K}^{\mathrm{S}}$ defines a potential value for a typical coupling for each conductive coupling existing in the model. For each non-zero $i j$ element of matrix $\mathbf{K}$, the conductive coupling between node $i$ and $j$ is defined as follows:

$$
K_{i j}^{S}=\frac{1}{\frac{L_{i}}{\lambda_{i} S_{i}}+\frac{L_{j}}{\lambda_{j} S_{j}}},
$$

\footnotetext{
${ }^{7}$ http://www.scipy-lectures.org/advanced/scipy sparse/csr_matrix.html
} 
where $\lambda_{i}$ and $\lambda_{j}$ are the thermal conductivities, $L_{i}$ and $L_{j}$ the distances between the node centers and the interface, and $S_{i}$ and $S_{j}$ the cross section of nodes $i$ an $j$ for the conductive coupling calculation. Assuming prismatic geometries, all the involved geometric magnitudes are shown in Fig. 4-3.

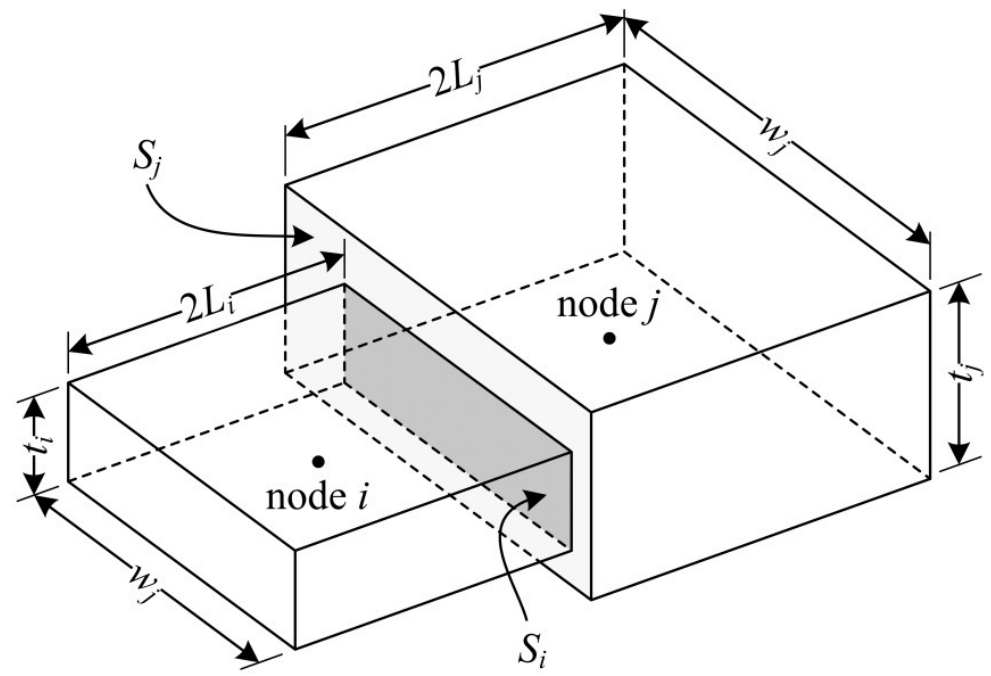

Fig. 4-3. Conductive coupling calculation scheme.

The thermal conductivities $\lambda_{i}$ are not explicitly included in the inputs mentioned in section 3 , so they are unknown. To estimate the sizing matrix $\mathbf{K}^{\mathrm{S}}$, a reference value, say $\lambda$, is used for all the conductors. This generic value itself does not matter and does not influence the results. It is intended just to obtain values with thermal engineering meaning. Therefore:

$$
K_{i j}^{S}=\frac{\lambda}{\frac{L_{i}}{S_{i}}+\frac{L_{j}}{S_{j}}} .
$$

As $S_{i}, S_{j}$ are also unknown, they are estimated as follows. Let us consider the thermal capacities for the nodes $i$ and $j$ :

$$
\left\{\begin{array}{l}
C_{i}=\rho_{i} c_{i} v_{i}=\rho_{i} c_{i} w_{i} t_{i} 2 L_{i} \\
C_{j}=\rho_{j} c_{j} v_{j}=\rho_{j} c_{j} w_{j} t_{j} 2 L_{j}
\end{array},\right.
$$

where, as for the thermal conductivities, $\rho$ and $c$ cannot be inferred from the input data. Again, standard values are used for these magnitudes. $S_{i}$ and $S_{j}$ can be defined as

$$
S_{i}=w_{i} t_{i} ; \quad S_{j}=w_{j} t_{j} .
$$

Although $w$ and $t$ are unknown magnitudes, they can be obtained from (26) as follows

$$
w_{i} t_{i}=\frac{C_{i}}{2 \rho c L_{i}} ; \quad w_{j} t_{j}=\frac{C_{j}}{2 \rho c L_{j}} .
$$


Substituting (27) and (28) into (25):

$$
K_{i j}^{s}=\frac{\lambda}{\frac{L_{i}}{w_{i} t_{i}}+\frac{L_{j}}{w_{j} t_{j}}}=\frac{\lambda}{2 \rho c} \frac{1}{\frac{L_{i}^{2}}{C_{i}}+\frac{L_{j}^{2}}{C_{j}}} .
$$

We can try to estimate the values for $L_{i}$ and $L_{j}$. From nodal spatial coordinates matrix, $\mathbf{X}$, the distance between nodal centers $i$ and $j, D_{i j}$, can be calculated. In $\mathbf{X}$, the first column corresponds to coordinate $x$, second column to $y$, and third to $z$. The distances are calculated as:

$$
D_{i j}=\sqrt{\left(x_{j}-x_{i}\right)^{2}+\left(y_{j}-y_{i}\right)^{2}+\left(z_{j}-z_{i}\right)^{2}} .
$$

It is assumed that the nodal geometries are of the same order of magnitude, and so will be the distances $L_{i}$ and $L_{j}$. Then

$$
L_{i} \sim L_{j} \rightarrow L_{i}=L_{j}=\frac{D_{i j}}{2}
$$

And consequently, using (29) and (31) we get

$$
K_{i j}^{S}=\frac{2 \lambda}{\rho c} \frac{1}{D_{i j}^{2}} \frac{1}{\frac{1}{C_{i}}+\frac{1}{C_{j}}}=\mu \frac{G_{i j}}{D_{i j}^{2}},
$$

where the parameter $\mu$ is defined as

$$
\mu=\frac{2 \lambda}{\rho c}
$$

and

$$
G_{i j}=\frac{1}{\frac{1}{C_{i}}+\frac{1}{C_{j}}} .
$$

The sizing matrix $\mathbf{K}^{\mathrm{S}}$ is obtained from (29) by calculating the thermal capacity and distance matrices, $\mathbf{G}$ and $\mathbf{D}$, which are symmetric, together with the parameter $\mu$. The choice of the parameter $\mu$ does not affect the construction of the dimensionless matrix $\tilde{\mathbf{K}}$, as the same value is used for all the elements. However, it is important to choose a realistic value, so that the sizing conductive coupling matrix $\mathbf{K}^{\mathrm{S}}$ has realistic values as well. Assuming typical values, such as $\lambda=50 \mathrm{~W} /(\mathrm{m} \cdot \mathrm{K}), \rho=3000 \mathrm{~kg} / \mathrm{m}^{3}$ and $c=1000 \mathrm{~J} /(\mathrm{kg} \cdot \mathrm{K})$, these would yield a value for $\mu=3.33 \cdot 10^{-5} \mathrm{~m}^{2} / \mathrm{s}$.

Once the sizing matrix $\mathbf{K}^{\mathrm{S}}$ has been calculated, the dimensionless matrix $\tilde{\mathbf{K}}$ is obtained by dividing element-wise the matrix $\mathbf{K}$ by $\mathbf{K}^{\mathrm{S}}$ (only applies for non-zero elements)

$$
\tilde{K}_{i j}=\frac{K_{i j}}{K_{i j}^{S}}, \quad i, j: K_{i j}^{S} \quad 0, \quad i \quad j .
$$


$\tilde{\mathbf{K}}$ represents the comparison between the conductive couplings of the detailed model and their estimated values obtained from the available data, such as geometry and thermal capacities.

\section{Exception handling}

The policy in the reduction process is to leave the nodes with insufficient information not condensed in the reduced model. We will call these nodes "isolated nodes". This is equivalent to make all the related conductors equal to zero in $\mathbf{K}$.

As a consequence of using ESATAN output, an specific procedure should be applied in some cases. In this software, all the elements with no spatial coordinates defined in the modelling process are located at the origin in the TMD output file (thus, showing the coordinates $[0,0,0]$ ). For a node $i$, all the distances $D_{i j}$ are set to zero, in the understanding that the position of the node $i$ is unknown, and therefore is impossible to assess the corresponding terms in $\mathbf{K}^{\mathrm{S}}$ and $\tilde{\mathbf{K}}$.

In the calculation of the thermal capacities matrix elements (34), if one of the capacities is zero, then the capacity term in the matrix will be the non-zero capacity. If both capacities are zero, then this term is not determined, and the capacity term is set to zero. The zeros in matrices $\mathbf{G}$ and $\mathbf{D}$ are propagated to $\mathbf{K}^{\mathrm{S}}$ and $\tilde{\mathbf{K}}$ by using CSR data operations.

\subsubsection{Boundary nodes processing}

The boundary nodes have to be retained in the reduced model, and should not be condensed. They should remain as isolated nodes (as only connected nodes are candidates for condensation). To achieve this, they have to be artificially decoupled from the rest of the nodes in the model in order to process the adjacency matrix $\mathbf{A}$ adequately. To do so, the boundary nodes in the sizing matrix $\tilde{\mathbf{K}}$ need to be marked $^{8}$. Basically, this means that all the related conductive couplings to the boundary nodes will be artificially set to zero, so that they are not able to be condensed with any other node (the boundary nodes appear as if they were isolated). It is important to underline that this is done only in the $\tilde{\mathbf{K}}$ matrix (the resulting matrix is called $\tilde{\mathbf{K}}^{\mathrm{B}}$ ). It only affects the reduction scheme, but all the information regarding the conductive couplings between the boundary nodes and rest of the model is still available, and will be consequently considered in the reduced model.

Furthermore, in order to improve the processing of the conductive coupling matrix and the outcome of the model reduction, it is useful to retain the nodes (mark them as isolated in $\tilde{\mathbf{K}}^{\mathrm{B}}$ ) that are directly conductively coupled to the boundary nodes. These nodes are called "internal boundary nodes". Regardless of the aim of the model reduction -intensive internal calculations, delivery to an assembler of thermal models, etc.-, the retention (isolation) of these internal nodes will generally allow for a better temperature correlation to be achieved in these nodes, which ultimately will yield a better heat fluxes correlation, because the conductive couplings between the boundary nodes and the internal boundary nodes are kept independent from the

\footnotetext{
${ }^{8}$ Due to operations sequence, the boundary and the internal boundary nodes are actually processed in a copy of the conductive couplings matrix $\mathbf{K}$. The nonzero elements in the resulting matrix become 1, and then this matrix is multiplied element-wise by $\tilde{\mathbf{K}}$, obtaining $\tilde{\mathbf{K}}^{1}$ (see paragraph 4.2.3).
} 
general reduction process. The process is applied in $\tilde{\mathbf{K}}^{\mathrm{B}}$, and the resulting matrix is called $\tilde{\mathbf{K}}^{\mathrm{I}}$. An scheme of the "isolation" process is shown in Fig. 4-4.

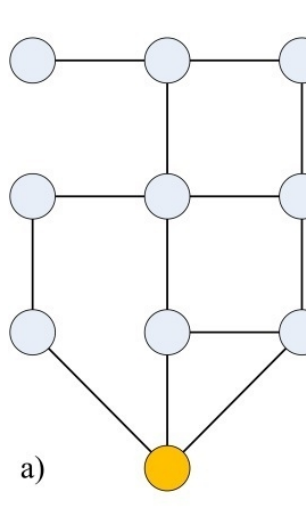

Boundary node
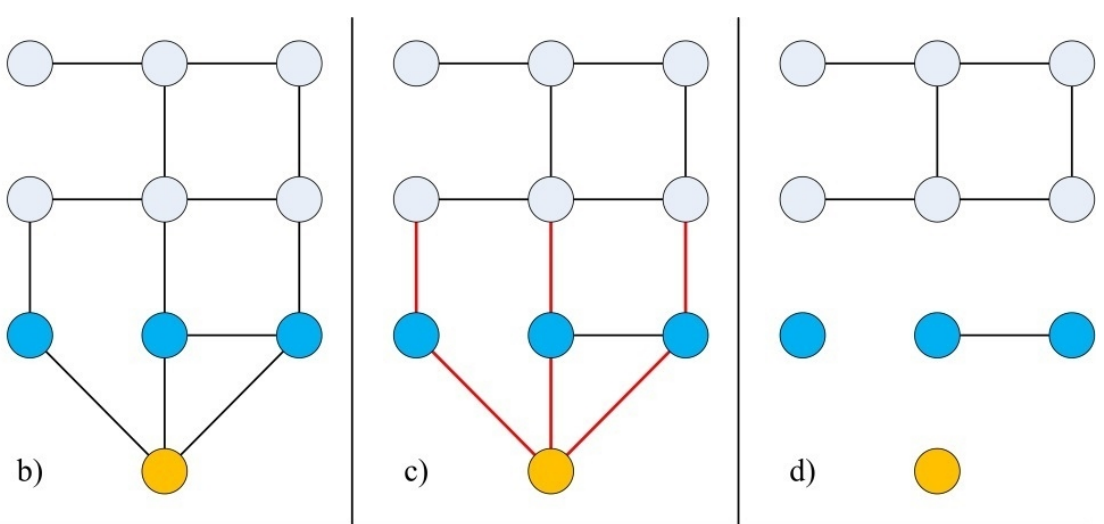

Diffusive node

Internal boundary node

Fig. 4-4. Boundary nodes and internal boundary nodes retention process. a) Shows nodes interconnected, including boundary nodes, in orange. b) Internal boundary nodes are identified, in blue. c) Isolation of boundary and internal boundary nodes, by removing conductive couplings, in red. d) Final state of the nodes, once the nodes have been isolated.

It has to be pointed out that the only purpose of isolating the nodes is to prevent them from participating in the condensation. The node or group of nodes is marked in $\tilde{\mathbf{K}}$ or $\tilde{\mathbf{K}}^{\mathrm{B}}$-are disconnected from its neighbors- in order to prevent them from being condensed with the nodes from which they have been disconnected. Once the model is reduced, the conductive couplings are recovered, and the nodes are not isolated anymore.

Regarding the boundary nodes that are connected to the rest of the model only by means of radiative couplings, they are already conductively disconnected, as their conductive couplings with the model are obviously zero.

\subsubsection{Filtering of the dimensionless conductive coupling matrix}

The matrix $\tilde{\mathbf{K}}^{\mathrm{I}}$ is ready to be filtered by a value $p_{f}$, which represents a dimensionless threshold as follows

$$
\tilde{K}_{i j}^{I, F}=\left\{\begin{array}{lc}
1 & \tilde{K}_{i j}^{I}>p_{f} \\
0 & \tilde{K}_{i j}^{I} \leq p_{f}
\end{array} .\right.
$$

The resulting matrix $\tilde{\mathbf{K}}^{\mathrm{I}, \mathrm{F}}$ is "binarized", a matrix with only ones and zeros, which contains the information of the nodes that are to be merged. The number of reduced nodes depends on $p_{f}$ $\tilde{K}_{i j}^{I, F}=1$ means a good connection, and therefore the connected nodes have to be condensed in the same reduced node. As the threshold value $p_{f}$ increases, the number of "good" connections decreases, more nodes appear as isolated in the model, and consequently the number of nodes in the reduced model is larger. 


\subsection{Construction and process of temperature difference matrix}

The representativeness of the reduced model is evaluated by comparing the temperatures of the reduced model and of the detailed model. Therefore, if the nodes from the detailed model that form a reduced node are isothermal (or nearly isothermal), the reduced model will behave more similarly to the detailed one. Thus, it is reasonable to limit the temperature difference that two nodes can have in order for them to be included in the same reduced node.

\subsubsection{Building and filtering the temperature difference matrix}

The temperature difference matrix $\boldsymbol{\theta}$ is built with the nodal temperature vector $\mathbf{T}$ of the detailed model,

$$
\theta_{i j}=\left|T_{i}-T_{j}\right|
$$

The matrix $\boldsymbol{\Theta}$ is ready to be filtered by a maximum temperature difference, $\Delta T_{\max }$. The resulting matrix is "binarized" in the same fashion as explained in section 4.2.3, as follows,

$$
\theta_{i j}^{F}=\left\{\begin{array}{ll}
0 & \theta_{i j}>\Delta T_{\max } \\
1 & \theta_{i j} \leq \Delta T_{\max }
\end{array} .\right.
$$

Obviously, the greater the maximum allowed temperature difference $\Delta T_{\max }$, the lower the number of nodes in the reduced model.

\subsection{Model condensation}

\subsubsection{Definition of the adjacency matrix}

With $\tilde{\mathbf{K}}^{\mathrm{I}, \mathrm{F}}$ and $\boldsymbol{\Theta}^{\mathrm{F}}$ already built, it is necessary to merge both matrices in the adjacency matrix A. This is achieved by multiplying both matrices element-wise

$$
A_{i j}=\tilde{K}_{i j}^{I, F} \theta_{i j}^{F}
$$

where essentially the filtered dimensionless matrix $\tilde{\mathbf{K}}^{\mathrm{I}, \mathrm{F}}$ is restricted with $\boldsymbol{\Theta}^{\mathrm{F}}$. Because of the definition of $\tilde{\mathbf{K}}^{\mathrm{I}, \mathrm{F}}$ and $\boldsymbol{\Theta}^{\mathrm{F}}$ in equations (36) and (38), the adjacency matrix $\mathbf{A}$ will have only zeros and ones. It represents the thermal network as a graph, the nodes being the graph vertices and the conductive couplings the edges (see Fig. 4-5). The thermal network is an undirected graph, and $\mathbf{A}$ is a sparse adjacency symmetrical matrix. As mentioned in section 4.1, the programming has been carried out using sparse matrices in CSR format [68]. 


$$
\mathbf{K}=\left(\begin{array}{llllllllll}
0 & 0 & 0 & 0 & 0 & 4 & 0 & 0 & 0 & 2 \\
0 & 0 & 8 & 0 & 0 & 0 & 0 & 0 & 3 & 0 \\
0 & 8 & 0 & 0 & 1 & 0 & 4 & 0 & 2 & 0 \\
0 & 0 & 0 & 0 & 0 & 0 & 0 & 0 & 0 & 2 \\
0 & 0 & 1 & 0 & 0 & 0 & 0 & 2 & 0 & 0 \\
4 & 0 & 0 & 0 & 0 & 0 & 0 & 4 & 0 & 1 \\
0 & 0 & 4 & 0 & 0 & 0 & 0 & 2 & 0 & 0 \\
0 & 0 & 0 & 0 & 2 & 4 & 2 & 0 & 0 & 2 \\
0 & 3 & 2 & 0 & 0 & 0 & 0 & 0 & 0 & 0 \\
2 & 0 & 0 & 2 & 0 & 1 & 0 & 2 & 0 & 0
\end{array}\right) \quad \mathbf{A}=\left(\begin{array}{llllllllll}
0 & 0 & 0 & 0 & 0 & 1 & 0 & 0 & 0 & 1 \\
0 & 0 & 1 & 0 & 0 & 0 & 0 & 0 & 1 & 0 \\
0 & 1 & 0 & 0 & 1 & 0 & 1 & 0 & 1 & 0 \\
0 & 0 & 0 & 0 & 0 & 0 & 0 & 0 & 0 & 1 \\
0 & 0 & 1 & 0 & 0 & 0 & 0 & 1 & 0 & 0 \\
1 & 0 & 0 & 0 & 0 & 0 & 0 & 1 & 0 & 1 \\
0 & 0 & 1 & 0 & 0 & 0 & 0 & 1 & 0 & 0 \\
0 & 0 & 0 & 0 & 1 & 1 & 1 & 0 & 0 & 1 \\
0 & 1 & 1 & 0 & 0 & 0 & 0 & 0 & 0 & 0 \\
1 & 0 & 0 & 1 & 0 & 1 & 0 & 1 & 0 & 0
\end{array}\right)
$$

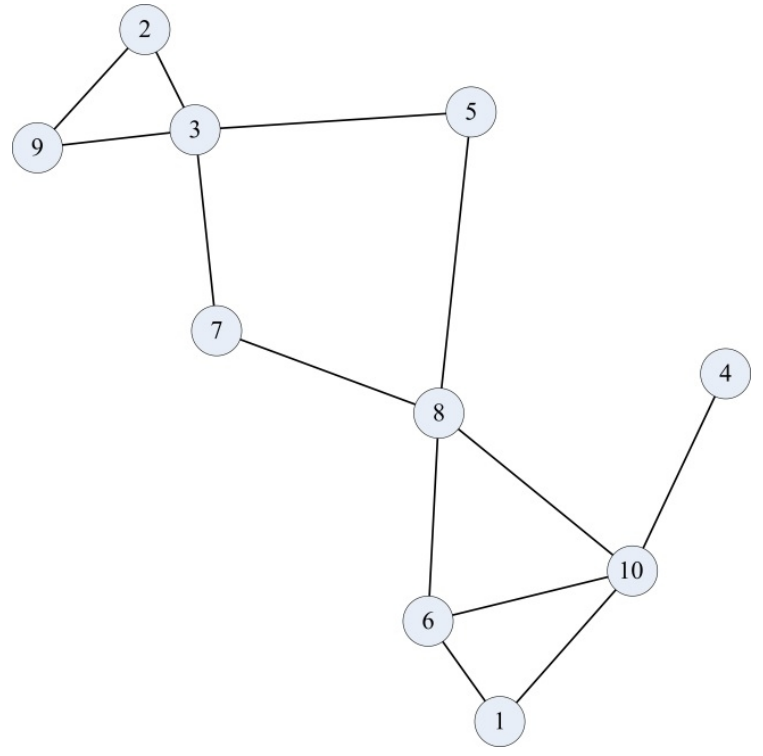

Fig. 4-5. Example conductive couplings matrix (K) on the top left (integer values for the sake of matrix compactness), the adjacency matrix A (after binarizing $K$ ) on the top right and the corresponding graph on the bottom.

\subsubsection{Connected components algorithm}

The model is reduced by running the connected components algorithm ${ }^{9}[60,62-64]$, so that the nodes are condensed according to their connections (the algorithm finds the nodes which are connected to each other). As a result, the algorithm returns the number of reduced nodes $n_{c}$, and the connected component labels array $\mathbf{S}_{\mathrm{cn}}$, in which the nodes belonging to the same connected component (thus, belonging to the same reduced node) have the same identifier.

\footnotetext{
${ }^{9}$ The routine used belongs to the SciPy Compressed Sparse Graphs package and can be looked up in https://docs.scipy.org/doc/scipy/reference/generated/scipy.sparse.csgraph.connected components.html
} 


\subsubsection{Sorting the matrix entities}

Sorting the original $\mathbf{K}$ and $\mathbf{R}$ matrices is not strictly necessary for the reduction process. However, arranging the thermal coupling matrices by reduced nodes order can give visual information about how the reduction process has grouped the nodes. To do this, a change of basis matrix $\mathbf{B}$ is necessary, which is built making use of the label array $\mathbf{S}_{\mathrm{cn}}$. Then, one can get the sorted versions of $\mathbf{K}$ and $\mathbf{R}$ as the following matrix products

$$
\left\{\begin{array}{l}
\mathbf{K}_{\text {sort }}=\mathbf{B} \mathbf{K} \mathbf{B}^{\mathrm{T}} \\
\mathbf{R}_{\text {sort }}=\mathbf{B} \mathbf{R} \mathbf{B}^{\mathrm{T}}
\end{array} .\right.
$$

(See example in Section 5.2.1).

\subsubsection{Compacting the model}

To reduce the dimension of the input matrices and vectors a transformation is required, which is performed by using the so-called restriction matrix $\mathbf{P}$. The dimensions of $\mathbf{P}$ are $n_{d}$ rows $\times n_{c}$ columns. The node order of $\mathbf{P}$ is the same as in $\mathbf{K}$, each row corresponds to a detailed node, which will have a " 1 " in the column corresponding to the associated condensed node, and the rest of the elements in the row will be " 0 ". With the restriction matrix, and the original inputs, the matrix entities can be built for the reduced model

$$
\left\{\begin{array}{l}
\mathbf{K}^{\mathrm{R}}=\mathbf{P}^{\mathrm{T}} \mathbf{K} \mathbf{P} \\
\mathbf{R}^{\mathrm{R}}=\mathbf{P}^{\mathrm{T}} \mathbf{R} \mathbf{P}
\end{array} .\right.
$$

Thus, for each condensed node, all of its conductive couplings with a neighbor are summed up. Same methodology is used for the radiative couplings. Actually, these combinations are the source of the deviation between the reduced nodes predicted temperatures (derived from the detailed thermal model) and those calculated by solving the reduced thermal model. The errors will be small as far as the condensed nodes are isothermal. Thus, the threshold values $\left(p_{f}\right.$ and $\Delta T_{\max }$ ) determine the error obtained in the reduction, considering the rest of the elements constant. An example of the formation of the reduced nodes and the condensation of the couplings among the nodes is shown in Fig. 4-6. 


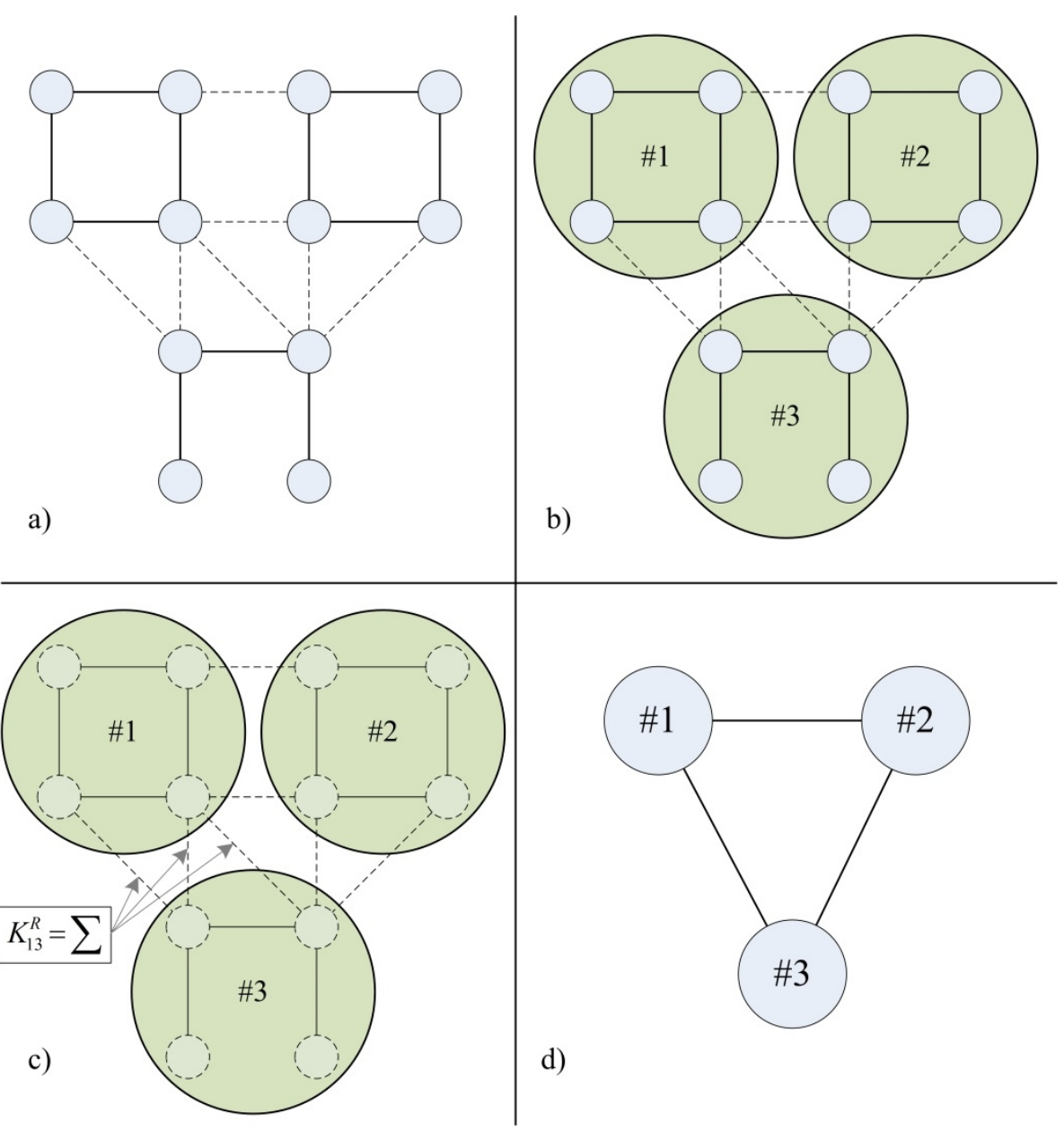

Fig. 4-6. Connected components and couplings reduction process. a) Strong conductive couplings among the nodes (solid black lines) and weak connections (dashed black lines). b) The connected components are found within the mesh, condensing the nodes in reduced nodes. Strong couplings vanish among the condensed nodes. c) The weak couplings are summed up to find the equivalent couplings between two reduced nodes. d) Final status of the reduced model.

The procedure of calculating the reduced model conductive couplings is shown in Fig. 4-6 c). The conductive couplings between detailed model nodes belonging to reduced node \#1 and those belonging to reduced node \#3 are summed up, in order to find the equivalent conductive coupling for the reduced model. The procedure is repeated for the rest of the model.

Also for the loads and thermal capacities vectors

$$
\begin{aligned}
& \mathbf{Q}^{\mathrm{R}}=\mathbf{P}^{\mathrm{T}} \mathbf{Q} \\
& \mathbf{C}^{\mathrm{R}}=\mathbf{P}^{\mathrm{T}} \mathbf{C}
\end{aligned}
$$

This operation assigns to each reduced node the sum of the thermal capacities of the nodes that are condensed into that reduced node. The same occurs for the thermal loads. The reduced system can be solved with the reduced matrices and vectors to obtain $\hat{\mathbf{T}}^{\mathrm{R}}$. However, the correctness or accuracy of the reduction process needs to be evaluated. Thus, this matrix need to be compared with the target temperatures $\overline{\mathbf{T}}^{\mathrm{D}}$, derived directly from the detailed model. One widely accepted standard in the industry is to calculate these temperatures as the thermal 
capacity-weighted mean temperature values [69] (it would be also possible to weight the temperatures with nodal areas)

$$
\bar{T}_{i}{ }^{D}=\frac{\sum_{j=1}^{m} T_{j} C_{j}}{\sum_{j=1}^{m} C_{j}}, i=1, \ldots, n_{c},
$$

where $m$ is the number of nodes that belong to reduced node $i$. It might happen that some elements belonging to a reduced node have zero thermal capacity. These nodes are called "arithmetic nodes", corresponding to elements with a very small mass, and they respond immediately to the changes in their environment $[16,70]$. The arithmetic nodes are ignored in the calculation of thermal capacity-weighted mean temperature values. If all the nodes condensed into a reduced node have zero thermal capacity, then the corresponding value in $\overline{\mathbf{T}}^{\mathrm{D}}$ is calculated as an arithmetic mean (this general rule works well for the boundary nodes, since they are the only element in their reduced nodes). Once the reduced model is solved, the temperatures derived from the detailed model, $\overline{\mathbf{T}}^{\mathrm{D}}$, are compared with those calculated with the reduced model, $\hat{\mathbf{T}}^{\mathrm{R}}$. The boundary nodes are directly assigned a temperature in the reduced model, as they were isolated in the adjacency matrix (see section 4.2.2).

Regarding the heat fluxes, the calculated values for the reduced model, $\hat{\mathbf{H}}^{\mathrm{R}}$, are compared with the target values, those corresponding to the detailed model, $\mathbf{H}_{\mathrm{cb}}^{\mathrm{D}}$ and $\mathbf{H}_{\mathrm{rb}}^{\mathrm{D}} \cdot \mathbf{H}_{\mathrm{cb}}^{\mathrm{D}}$ accounts for the conductive heat fluxes to boundary nodes, whereas the vector $\mathbf{H}_{\mathrm{rb}}^{\mathrm{D}}$ does so for the radiative heat fluxes.

\subsection{Validation of the reduced thermal model}

With the reduced matrices and vectors, the reduced system can be solved. The derived temperatures vector $\overline{\mathbf{T}}^{\mathrm{D}}$ is used to set up the boundary conditions, and also as an initial condition if an iterative solver is employed. The thermal system is then solved in steady state conditions, one energy balance equation per node

$$
\sum_{j=1}^{n_{c}}\left[K_{i j}^{R}\left(\hat{T}_{i}^{R}-\hat{T}_{j}^{R}\right)+R_{i j}^{R}\left(\hat{T}_{i}^{R^{4}}-\hat{T}_{j}^{R^{4}}\right)\right]+Q_{i}^{R}=0, \quad i=1, \ldots, n_{c}, \quad i \quad j .
$$

The model is solved with a steady state solver. For the linearization of the radiative terms, the following algebraic identity is used (see [71])

$$
\left(\hat{T}_{i}^{R^{4}}-\hat{T}_{j}^{R^{4}}\right)=\left(\hat{T}_{i}^{R^{2}}+\hat{T}_{j}^{R^{2}}\right)\left(\hat{T}_{i}^{R}+\hat{T}_{j}^{R}\right)\left(\hat{T}_{i}^{R}-\hat{T}_{j}^{R}\right) .
$$

Using eq. (45) in the nonlinear part of eq. (44), one gets

$$
R_{i j}^{R}\left(\hat{T}_{i}^{R^{4}}-\hat{T}_{j}^{R^{4}}\right)=R_{i j}^{R}\left(\hat{T}_{i}^{R^{2}}+\hat{T}_{j}^{R^{2}}\right)\left(\hat{T}_{i}^{R}+\hat{T}_{j}^{R}\right)\left(\hat{T}_{i}^{R}-\hat{T}_{j}^{R}\right)=R_{i j}^{R^{*}}\left(\hat{T}_{i}^{R}-\hat{T}_{j}^{R}\right),
$$

where

$$
R_{i j}^{R^{*}}=\left(\hat{T}_{i}^{R^{2}}+\hat{T}_{j}^{R^{2}}\right)\left(\hat{T}_{i}^{R}+\hat{T}_{j}^{R}\right)
$$


The values for $\mathbf{K}^{\mathrm{R}^{*}}$ are obtained with the temperatures calculated for the current step. Then, the system is solved, and the latest temperatures are compared with those corresponding to the previous step. Once the model has been solved, the results must be compared with those coming from the detailed model. For the temperatures, the absolute difference will have to be less than a predefined value, $\delta_{\max }$. Hence, with the reduced model derived temperatures $\overline{\mathbf{T}}^{\mathrm{D}}$, and the reduced model calculated temperatures $\hat{\mathbf{T}}^{\mathrm{R}}$, it can be checked that the difference is below the maximum allowable value $\delta_{\max }$.

$$
\delta_{i}=\left|\bar{T}_{i}^{D}-\hat{T}_{i}^{R}\right|<\delta_{\max }, \quad i=1, \ldots, n_{c} .
$$

The difference between the temperature of each node in the detailed model and its corresponding calculated temperature of the reduced node, to which the original node belongs, can be calculated as

$$
\boldsymbol{\tau}=\mathbf{T}-\hat{\mathbf{T}}_{\mathrm{d}}^{\mathrm{R}}=\mathbf{T}-\left(\mathbf{P} \hat{\mathbf{T}}^{\mathrm{R}}\right) .
$$

Regarding the heat fluxes exchanged with the boundary nodes, the heat fluxes vectors will have as many components as the number of boundary nodes, $n_{b}$. The difference can be expressed in terms of percentage or in absolute numbers, depending on the heat flux value (the limit is defined by $q_{\text {lim }}$ ). For the conductive heat fluxes

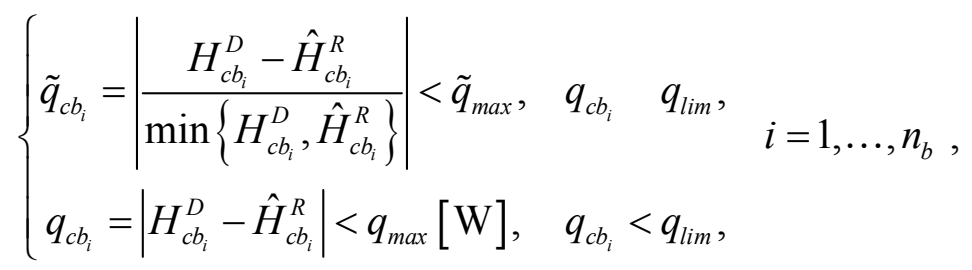

and for the radiative heat fluxes

$$
\left\{\begin{array}{l}
\tilde{q}_{r b_{i}}=\left|\frac{H_{r b_{i}}^{D}-\hat{H}_{r b_{i}}^{R}}{\min \left\{H_{r b_{i}}^{D}, \hat{H}_{r b_{i}}^{R}\right\}}\right|<\tilde{q}_{m a x}, \quad q_{r b_{i}} \quad q_{\text {lim }}, \quad i=1, \ldots, n_{b}, \\
q_{r b_{i}}=\left|H_{r b_{i}}^{D}-\hat{H}_{r b_{i}}^{R}\right|<q_{\max }[\mathrm{W}], \quad q_{r b_{i}}<q_{\text {lim }} .
\end{array}\right.
$$

The model reduction mathematical operations finish with the calculation of the correlation errors. The reduced model obtained depends on the values of the thresholds chosen $\left(p_{f}\right.$ and $\Delta T_{\max }$ ). More iterations can be run using different values of $p_{f}$ and $\Delta T_{\max }$. The problem of implementing an automatic procedure to find a pair of threshold values that optimize a certain objective function is beyond the scope of this work. For the time being, the final choice for the thresholds relies on the thermal engineer judgement. A parametric sweep that explores many different possible combinations of $p_{f}$ and $\Delta T_{\max }$ is shown in section 5.2. This sweep can help the engineer to study the problem and pick a suitable combination of thresholds for the thermal model reduction. 


\section{TESTING OF THE PROPOSED METHODOLOGY}

Two models are used in order to test the methodology described in chapter 4 . The first one, a 10-node model, will allow us to write down the matrices and vectors as the reduction process develops. The second model represents real space hardware and will test quantitatively the reduction algorithm.

\subsection{0-node test model}

The 10-node model used for reduction method illustration is shown in Fig. 5-1. This small model represents a small housing, with some equipment inside. The equipment is conductively coupled to the lateral faces of the housing (lateral wall 1, Lat1, is not shown in the figure). The base is meshed forming two nodes, which are connected to the platform. Everything is surrounded by a boundary node, the so-called environment, which is not represented in the figure.

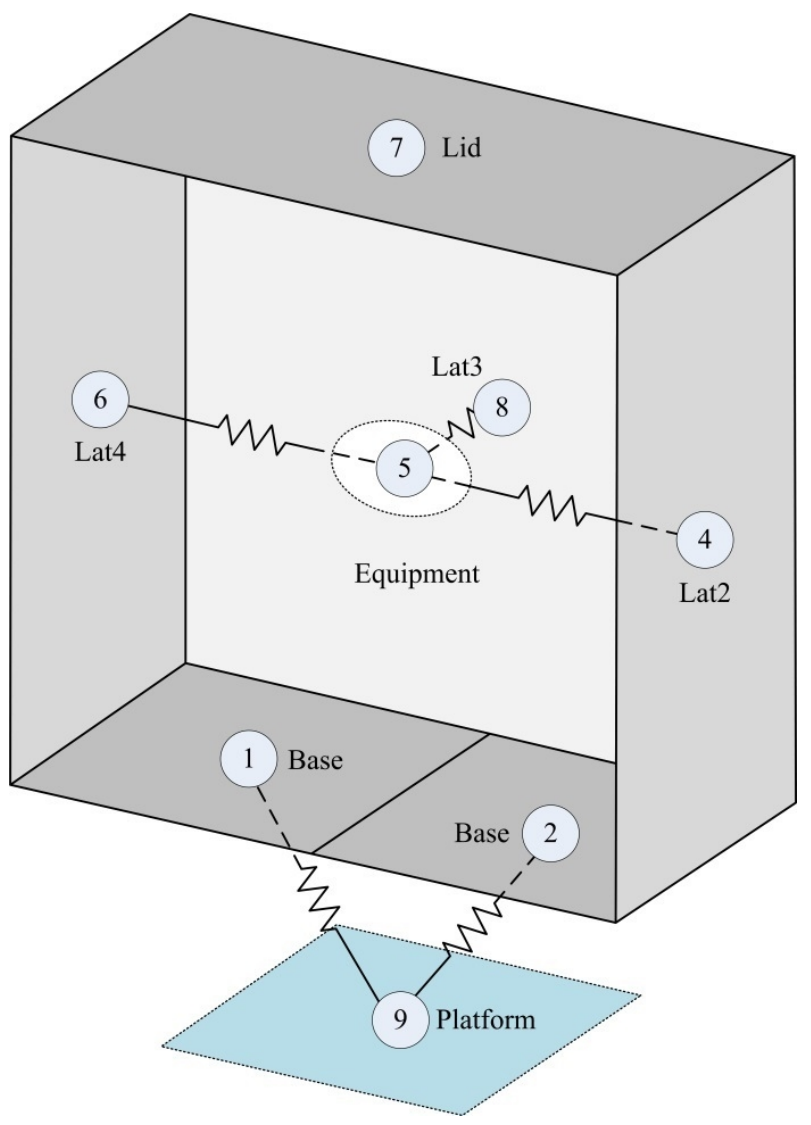

Fig. 5-1. 10-node model sketch (node 3 not shown).

The housing forms a rectangular prism, whose size is $5 \times 10 \times 10 \mathrm{~cm}^{3}$, and is made of aluminum, with a thickness of $2 \mathrm{~mm}$, painted black on both sides. The equipment is modelled as a non-geometrical node, with a thermal capacity of $100 \mathrm{~J} / \mathrm{K}$. The conductive couplings are shown in Fig. 5-2. 


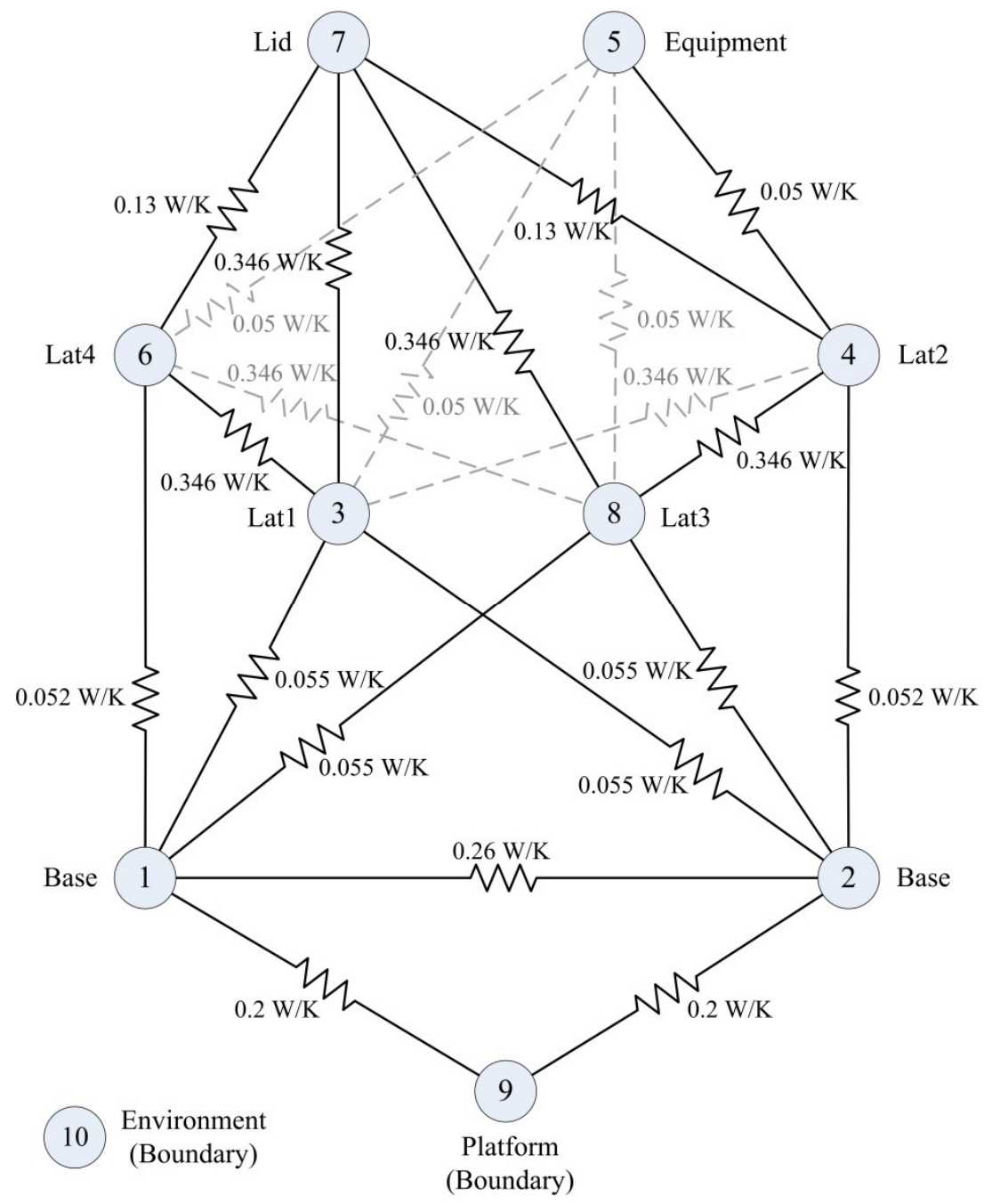

Fig. 5-2. Sketch of 10-node model conductive couplings. There is no difference between solid black and dashed grey lines in the figure. Grey dashed lines have been used solely for visualization purposes.

The system is defined by the following matrices and vectors

$$
\mathbf{K}=\left(\begin{array}{cccccccccc}
0 & 0.26 & 0.06 & 0 & 0 & 0.05 & 0 & 0.06 & 0.20 & 0 \\
0 & 0 & 0.06 & 0.05 & 0 & 0 & 0 & 0.06 & 0.20 & 0 \\
0 & 0 & 0 & 0.35 & 0.05 & 0.35 & 0.35 & 0 & 0 & 0 \\
0 & 0 & 0 & 0 & 0.05 & 0 & 0.13 & 0.35 & 0 & 0 \\
0 & 0 & 0 & 0 & 0 & 0.05 & 0 & 0.05 & 0 & 0 \\
0 & 0 & 0 & 0 & 0 & 0 & 0.13 & 0.35 & 0 & 0 \\
0 & 0 & 0 & 0 & 0 & 0 & 0 & 0.35 & 0 & 0 \\
0 & 0 & 0 & 0 & 0 & 0 & 0 & 0 & 0 & 0 \\
0 & 0 & 0 & 0 & 0 & 0 & 0 & 0 & 0 & 0 \\
0 & 0 & 0 & 0 & 0 & 0 & 0 & 0 & 0 & 0
\end{array}\right) \mathrm{W} / \mathrm{K}
$$




$$
\mathbf{R}=\left(\begin{array}{cccccccccc}
0 & 0.01 & 0.64 & 0.15 & 0 & 0.51 & 0.25 & 0.65 & 0 & 2.25 \\
0 & 0 & 0.64 & 0.50 & 0 & 0.15 & 0.25 & 0.65 & 0 & 2.25 \\
0 & 0 & 0 & 1.30 & 0 & 1.28 & 1.29 & 3.52 & 0 & 9.00 \\
0 & 0 & 0 & 0 & 0 & 0.51 & 0.66 & 1.29 & 0 & 4.50 \\
0 & 0 & 0 & 0 & 0 & 0 & 0 & 0 & 0 & 0 \\
0 & 0 & 0 & 0 & 0 & 0 & 0.65 & 1.29 & 0 & 4.50 \\
0 & 0 & 0 & 0 & 0 & 0 & 0 & 1.30 & 0 & 4.50 \\
0 & 0 & 0 & 0 & 0 & 0 & 0 & 0 & 0 & 9.00 \\
0 & 0 & 0 & 0 & 0 & 0 & 0 & 0 & 0 & 0 \\
0 & 0 & 0 & 0 & 0 & 0 & 0 & 0 & 0 & 0
\end{array}\right) \times 10^{-3} \mathrm{~m}^{2}
$$

Although here dense representation of the matrices is shown, the program handles them generally using sparse types. Regarding the thermal capacity and heat loads:

$$
\mathbf{C}=\left[\begin{array}{c}
13.0 \\
13.0 \\
51.8 \\
25.9 \\
100.0 \\
25.9 \\
25.9 \\
51.8 \\
1000.0 \\
0
\end{array}\right] \mathrm{J} / \mathrm{K} ; \quad \mathbf{Q}=\left[\begin{array}{c}
0 \\
0 \\
0 \\
0 \\
10.0 \\
0 \\
0 \\
0 \\
0 \\
0
\end{array}\right] \mathrm{W}
$$

With the system defined as above, we can obtain the steady state solution solving equation (4), using as boundary conditions the temperatures in the platform $\left(+35^{\circ} \mathrm{C}\right)$ and the radiative environment $\left(+50{ }^{\circ} \mathrm{C}\right)$

$$
\mathbf{T}=\left[\begin{array}{c}
49.73 \\
49.73 \\
65.64 \\
66.61 \\
116.13 \\
66.61 \\
65.27 \\
65.64 \\
35.00 \\
50.00
\end{array}\right] \mathbf{C} ; \quad \mathbf{H}_{\mathrm{cb}}^{\mathrm{D}}=\left[\begin{array}{c}
5.89 \\
0
\end{array}\right] \mathrm{W} ; \quad \mathbf{H}_{\mathrm{rb}}^{\mathrm{D}}=\left[\begin{array}{c}
0 \\
4.11
\end{array}\right] \mathrm{W}
$$


If the two boundary nodes are isolated as explained in section 4.2.2, and we run the SCC algorithm -no filtering is done, SCC routine is run directly on $\mathbf{K}$ once the boundary nodes have been disconnected from the rest of the model- we obtain a model with three nodes, two out of which are boundary type. We can calculate the maximum reduction ratio using eq. (23): $r_{r}=0.875$. This number indicates the maximum reduction we could get with this methodology, taking into account that the internal boundary nodes are not being isolated.

Let us now make the conductive couplings matrix $\mathbf{K}$ dimensionless. For that, we need to build $\mathbf{K}^{\mathrm{S}}$ (24), and then divide element-wise $\mathbf{K}$ by $\mathbf{K}^{\mathrm{S}}$, to obtain $\tilde{\mathbf{K}}$. Again, we choose $\mu=3.33 \cdot 10^{-5} \mathrm{~m}^{2} / \mathrm{s}$. As detailed in equation (32), we calculate distance matrix $\mathbf{D}$ and thermal capacity matrix $\mathbf{G}$

$$
\begin{aligned}
& \mathbf{D}=\left(\begin{array}{cccccccccc}
0 & 0.05 & 0.06 & 0 & 0 & 0.06 & 0 & 0.06 & 0.04 & 0 \\
0 & 0 & 0.06 & 0.06 & 0 & 0 & 0 & 0.06 & 0.04 & 0 \\
0 & 0 & 0 & 0.06 & 0.02 & 0.06 & 0.06 & 0 & 0 & 0 \\
0 & 0 & 0 & 0 & 0.05 & 0 & 0.07 & 0.06 & 0 & 0 \\
0 & 0 & 0 & 0 & 0 & 0.05 & 0 & 0.02 & 0 & 0 \\
0 & 0 & 0 & 0 & 0 & 0 & 0.07 & 0.06 & 0 & 0 \\
0 & 0 & 0 & 0 & 0 & 0 & 0 & 0.06 & 0 & 0 \\
0 & 0 & 0 & 0 & 0 & 0 & 0 & 0 & 0 & 0 \\
0 & 0 & 0 & 0 & 0 & 0 & 0 & 0 & 0 & 0 \\
0 & 0 & 0 & 0 & 0 & 0 & 0 & 0 & 0 & 0
\end{array}\right) \mathrm{m} \\
& \mathbf{G}=\left(\begin{array}{cccccccccc}
0 & 6.48 & 10.37 & 0 & 0 & 8.64 & 0 & 10.37 & 12.79 & 0 \\
0 & 0 & 10.37 & 8.64 & 0 & 0 & 0 & 10.37 & 12.79 & 0 \\
0 & 0 & 0 & 17.28 & 34.14 & 17.28 & 17.28 & 0 & 0 & 0 \\
0 & 0 & 0 & 0 & 20.58 & 0 & 12.96 & 17.28 & 0 & 0 \\
0 & 0 & 0 & 0 & 0 & 20.58 & 0 & 34.14 & 0 & 0 \\
0 & 0 & 0 & 0 & 0 & 0 & 12.96 & 17.28 & 0 & 0 \\
0 & 0 & 0 & 0 & 0 & 0 & 0 & 17.28 & 0 & 0 \\
0 & 0 & 0 & 0 & 0 & 0 & 0 & 0 & 0 & 0 \\
0 & 0 & 0 & 0 & 0 & 0 & 0 & 0 & 0 & 0 \\
0 & 0 & 0 & 0 & 0 & 0 & 0 & 0 & 0 & 0
\end{array}\right) \mathrm{J} / \mathrm{K}
\end{aligned}
$$

These matrices are masked with matrix $\mathbf{K}$, so that no unnecessary elements are stored (this can be relevant for larger models). Using expression (32), we get $\mathbf{K}^{\mathrm{S}}$ 


$$
\mathbf{K}^{\mathrm{S}}=\left(\begin{array}{cccccccccc}
0 & 0.09 & 0.09 & 0 & 0 & 0.09 & 0 & 0.09 & 0.34 & 0 \\
0 & 0 & 0.09 & 0.09 & 0 & 0 & 0 & 0.09 & 0.34 & 0 \\
0 & 0 & 0 & 0.18 & 1.82 & 0.18 & 0.18 & 0 & 0 & 0 \\
0 & 0 & 0 & 0 & 0.27 & 0 & 0.09 & 0.18 & 0 & 0 \\
0 & 0 & 0 & 0 & 0 & 0.27 & 0 & 1.82 & 0 & 0 \\
0 & 0 & 0 & 0 & 0 & 0 & 0.09 & 0.18 & 0 & 0 \\
0 & 0 & 0 & 0 & 0 & 0 & 0 & 0.18 & 0 & 0 \\
0 & 0 & 0 & 0 & 0 & 0 & 0 & 0 & 0 & 0 \\
0 & 0 & 0 & 0 & 0 & 0 & 0 & 0 & 0 & 0 \\
0 & 0 & 0 & 0 & 0 & 0 & 0 & 0 & 0 & 0
\end{array}\right) \mathrm{W} / \mathrm{K}
$$

and dividing $\mathbf{K}$ by $\mathbf{K}^{\mathrm{S}}$ yields $\tilde{\mathbf{K}}$

$$
\tilde{\mathbf{K}}=\left(\begin{array}{cccccccccc}
0 & 3.01 & 0.60 & 0 & 0 & 0.57 & 0 & 0.60 & 0.59 & 0 \\
0 & 0 & 0.60 & 0.57 & 0 & 0 & 0 & 0.60 & 0.59 & 0 \\
0 & 0 & 0 & 1.88 & 0.03 & 1.88 & 1.88 & 0 & 0 & 0 \\
0 & 0 & 0 & 0 & 0.18 & 0 & 1.50 & 1.88 & 0 & 0 \\
0 & 0 & 0 & 0 & 0 & 0.18 & 0 & 0.03 & 0 & 0 \\
0 & 0 & 0 & 0 & 0 & 0 & 1.50 & 1.88 & 0 & 0 \\
0 & 0 & 0 & 0 & 0 & 0 & 0 & 1.88 & 0 & 0 \\
0 & 0 & 0 & 0 & 0 & 0 & 0 & 0 & 0 & 0 \\
0 & 0 & 0 & 0 & 0 & 0 & 0 & 0 & 0 & 0 \\
0 & 0 & 0 & 0 & 0 & 0 & 0 & 0 & 0 & 0
\end{array}\right)
$$

Elements with a value greater than 1 are supposedly better conductors than the "assumed" value, as explained in 4.2.1. For instance, the element $\tilde{K}_{12}$ represents the coupling between two nodes belonging to the same plate. The bulk material of the plate (aluminum, with higher conductivity than the $\lambda=50 \mathrm{~W} /(\mathrm{m} \cdot \mathrm{K})$ used for calculating $\mu$ ) and the slightly different density and specific heat (from those used for calculating $\mu$ ) make $\tilde{K}_{12}>1$. On the other hand, $\tilde{K}_{23}$ represents the coupling between two nodes belonging to the different plates. Although they are made of aluminum, their conductive coupling is defined by a contact, which lowers the resulting value in $K_{12}$.

The boundary nodes (nodes 9 and 10) can be isolated in the model (see 4.2.2), by making zero the corresponding elements in matrix $\tilde{\mathbf{K}}$. Only node 9 has conductive couplings with the rest of the model 


$$
\tilde{\mathbf{K}}^{\text {B }}=\left(\begin{array}{cccccccc|cc}
0 & 3.01 & 0.60 & 0 & 0 & 0.57 & 0 & 0.60 & 0 & 0 \\
0 & 0 & 0.60 & 0.57 & 0 & 0 & 0 & 0.60 & 0 & 0 \\
0 & 0 & 0 & 1.88 & 0.03 & 1.88 & 1.88 & 0 & 0 & 0 \\
0 & 0 & 0 & 0 & 0.18 & 0 & 1.50 & 1.88 & 0 & 0 \\
0 & 0 & 0 & 0 & 0 & 0.18 & 0 & 0.03 & 0 & 0 \\
0 & 0 & 0 & 0 & 0 & 0 & 1.50 & 1.88 & 0 & 0 \\
0 & 0 & 0 & 0 & 0 & 0 & 0 & 1.88 & 0 & 0 \\
0 & 0 & 0 & 0 & 0 & 0 & 0 & 0 & 0 & 0 \\
\hline 0 & 0 & 0 & 0 & 0 & 0 & 0 & 0 & 0 & 0 \\
\hline 0 & 0 & 0 & 0 & 0 & 0 & 0 & 0 & 0 & 0
\end{array}\right)
$$

Then, the internal boundary nodes can be isolated as well. Only nodes 1 and 2 are conductively coupled to a boundary node. Thus, these nodes are isolated from the rest of the model. Note that the coupling between the two internal boundary nodes 1 and 2 does not vanish.

$$
\tilde{\mathbf{K}}^{I}=\left(\begin{array}{cccccccccc}
0 & 3.01 & 0 & 0 & 0 & 0 & 0 & 0 & 0 & 0 \\
0 & 0 & 0 & 0 & 0 & 0 & 0 & 0 & 0 & 0 \\
0 & 0 & 0 & 1.88 & 0.03 & 1.88 & 1.88 & 0 & 0 & 0 \\
0 & 0 & 0 & 0 & 0.18 & 0 & 1.50 & 1.88 & 0 & 0 \\
0 & 0 & 0 & 0 & 0 & 0.18 & 0 & 0.03 & 0 & 0 \\
0 & 0 & 0 & 0 & 0 & 0 & 1.50 & 1.88 & 0 & 0 \\
0 & 0 & 0 & 0 & 0 & 0 & 0 & 1.88 & 0 & 0 \\
0 & 0 & 0 & 0 & 0 & 0 & 0 & 0 & 0 & 0 \\
0 & 0 & 0 & 0 & 0 & 0 & 0 & 0 & 0 & 0 \\
0 & 0 & 0 & 0 & 0 & 0 & 0 & 0 & 0 & 0
\end{array}\right)
$$

Choosing $p_{f}$ as 0.2 , we get the filtered dimensionless conductive coupling matrix $\tilde{\mathbf{K}}^{\mathrm{I} F}$ (see (36)) 


$$
\tilde{\mathbf{K}}^{\mathrm{I}, \mathrm{F}}=\left(\begin{array}{cccccccccc}
0 & 1 & 0 & 0 & 0 & 0 & 0 & 0 & 0 & 0 \\
0 & 0 & 0 & 0 & 0 & 0 & 0 & 0 & 0 & 0 \\
0 & 0 & 0 & 1 & 0 & 1 & 1 & 0 & 0 & 0 \\
0 & 0 & 0 & 0 & 0 & 0 & 1 & 1 & 0 & 0 \\
0 & 0 & 0 & 0 & 0 & 0 & 0 & 0 & 0 & 0 \\
0 & 0 & 0 & 0 & 0 & 0 & 1 & 1 & 0 & 0 \\
0 & 0 & 0 & 0 & 0 & 0 & 0 & 1 & 0 & 0 \\
0 & 0 & 0 & 0 & 0 & 0 & 0 & 0 & 0 & 0 \\
0 & 0 & 0 & 0 & 0 & 0 & 0 & 0 & 0 & 0 \\
0 & 0 & 0 & 0 & 0 & 0 & 0 & 0 & 0 & 0
\end{array}\right)
$$

With $p_{f}=0.2$, all the conductive couplings between node 5 (equipment) and the lateral panels of the housing are filtered. As mentioned in section 4.2.3, once the matrix has been filtered, all the nonzero elements are substituted by ones. Regarding temperature difference matrix $\boldsymbol{\Theta}$

$$
\boldsymbol{\theta}=\left(\begin{array}{cccccccccc}
0 & 0 & 15.9 & 16.9 & 66.4 & 16.9 & 15.5 & 15.9 & 14.7 & 0.3 \\
0 & 0 & 15.9 & 16.9 & 66.4 & 16.9 & 15.5 & 15.9 & 14.7 & 0.3 \\
0 & 0 & 0 & 1.0 & 50.5 & 1.0 & 0.4 & 0 & 30.6 & 15.6 \\
0 & 0 & 0 & 0 & 49.5 & 0 & 1.3 & 1.0 & 31.6 & 16.6 \\
0 & 0 & 0 & 0 & 0 & 49.5 & 50.9 & 50.5 & 81.1 & 66.1 \\
0 & 0 & 0 & 0 & 0 & 0 & 1.3 & 1.0 & 31.6 & 16.6 \\
0 & 0 & 0 & 0 & 0 & 0 & 0 & 0.4 & 30.3 & 15.3 \\
0 & 0 & 0 & 0 & 0 & 0 & 0 & 0 & 30.6 & 15.6 \\
0 & 0 & 0 & 0 & 0 & 0 & 0 & 0 & 0 & 15.0 \\
0 & 0 & 0 & 0 & 0 & 0 & 0 & 0 & 0 & 0
\end{array}\right) \text { C }
$$

Applying a temperature difference threshold of $10^{\circ} \mathrm{C}, \boldsymbol{\Theta}$ is filtered and we obtain $\boldsymbol{\Theta}^{\mathrm{F}}$. Again, all the nonzero elements are substituted by ones 


$$
\boldsymbol{\theta}^{\mathrm{F}}=\left(\begin{array}{llllllllll}
0 & 1 & 0 & 0 & 0 & 0 & 0 & 0 & 0 & 1 \\
0 & 0 & 0 & 0 & 0 & 0 & 0 & 0 & 0 & 1 \\
0 & 0 & 0 & 1 & 0 & 1 & 1 & 1 & 0 & 0 \\
0 & 0 & 0 & 0 & 0 & 1 & 1 & 1 & 0 & 0 \\
0 & 0 & 0 & 0 & 0 & 0 & 0 & 0 & 0 & 0 \\
0 & 0 & 0 & 0 & 0 & 0 & 1 & 1 & 0 & 0 \\
0 & 0 & 0 & 0 & 0 & 0 & 0 & 1 & 0 & 0 \\
0 & 0 & 0 & 0 & 0 & 0 & 0 & 0 & 0 & 0 \\
0 & 0 & 0 & 0 & 0 & 0 & 0 & 0 & 0 & 0 \\
0 & 0 & 0 & 0 & 0 & 0 & 0 & 0 & 0 & 0
\end{array}\right)
$$

We can multiply element-wise matrices $\tilde{\mathbf{K}}^{\mathrm{I}, \mathrm{F}}$ and $\mathbf{\Theta}^{\mathrm{F}}$ to get the adjacency matrix $\mathbf{A}$

$$
\mathbf{A}=\left(\begin{array}{llllllllll}
0 & 1 & 0 & 0 & 0 & 0 & 0 & 0 & 0 & 0 \\
0 & 0 & 0 & 0 & 0 & 0 & 0 & 0 & 0 & 0 \\
0 & 0 & 0 & 1 & 0 & 1 & 1 & 0 & 0 & 0 \\
0 & 0 & 0 & 0 & 0 & 0 & 1 & 1 & 0 & 0 \\
0 & 0 & 0 & 0 & 0 & 0 & 0 & 0 & 0 & 0 \\
0 & 0 & 0 & 0 & 0 & 0 & 1 & 1 & 0 & 0 \\
0 & 0 & 0 & 0 & 0 & 0 & 0 & 1 & 0 & 0 \\
0 & 0 & 0 & 0 & 0 & 0 & 0 & 0 & 0 & 0 \\
0 & 0 & 0 & 0 & 0 & 0 & 0 & 0 & 0 & 0 \\
0 & 0 & 0 & 0 & 0 & 0 & 0 & 0 & 0 & 0
\end{array}\right)
$$

By running the SCC routine on adjacency matrix $\mathbf{A}$, we can find the condensed nodes:

$$
n_{c}=5 ; \quad \mathbf{S}_{\mathrm{cn}}=\left[\begin{array}{llllllllll}
1 & 1 & 2 & 2 & 3 & 2 & 2 & 2 & 4 & 5
\end{array}\right]
$$

Vector $\mathbf{S}_{\mathrm{cn}}$ is one of the outputs of the connected components routine (see section 4.4.2). The vector has one component per detailed model node, indicating the reduced thermal model node number to which the detailed node belongs. The adjacency matrix $\mathbf{A}$, together with the corresponding reduced model node numbers, is shown below: 


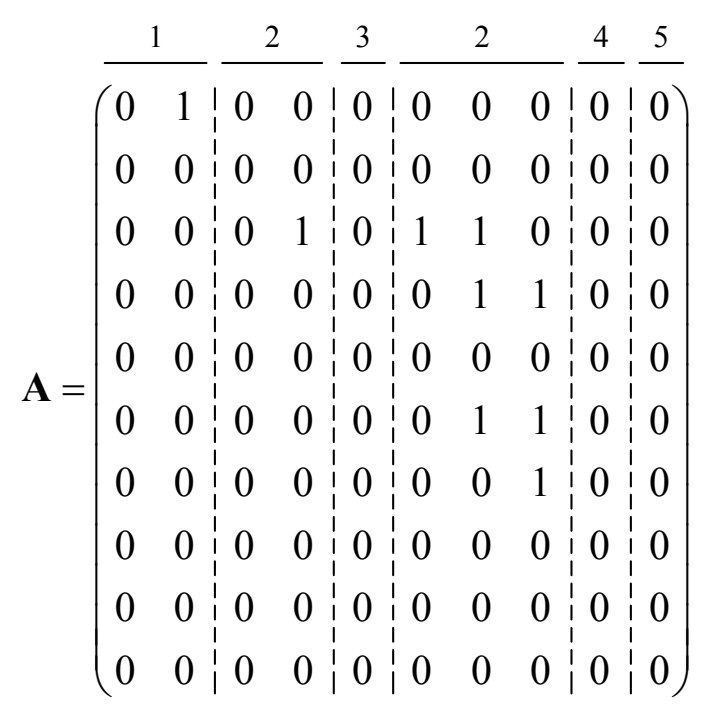

The reduced model has 5 reduced nodes, so $r_{r}=0.62$. Looking at $\mathbf{S}_{\mathrm{cn}}$, we can see that the first two nodes (base) belong to reduced node No.1. Then we have the housing laterals and lid forming another reduced node (No.2), the equipment (No.3) and the two boundary nodes (No.4 and No.5). Fig. 5-3 shows the 10-node model with the reduced nodes. 


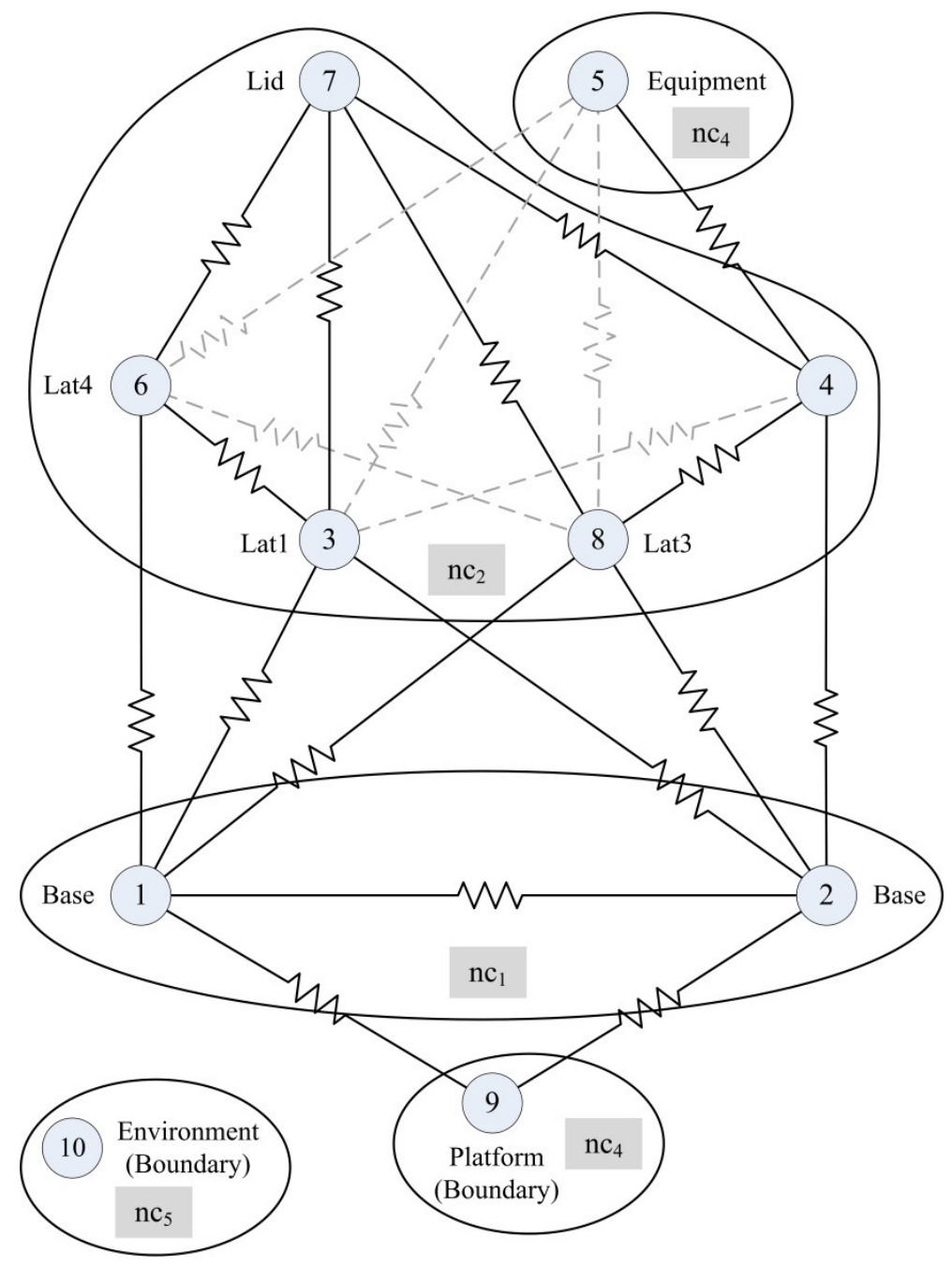

Fig. 5-3. Sketch of 10-node model with the condensed nodes.

In order to sort the conductive and radiative couplings matrices by the condensed nodes, it is necessary to build a change-of-basis matrix $\mathbf{B}$

$$
\mathbf{B}=\left(\begin{array}{llllllllll}
1 & 0 & 0 & 0 & 0 & 0 & 0 & 0 & 0 & 0 \\
0 & 1 & 0 & 0 & 0 & 0 & 0 & 0 & 0 & 0 \\
0 & 0 & 1 & 0 & 0 & 0 & 0 & 0 & 0 & 0 \\
0 & 0 & 0 & 1 & 0 & 0 & 0 & 0 & 0 & 0 \\
0 & 0 & 0 & 0 & 0 & 1 & 0 & 0 & 0 & 0 \\
0 & 0 & 0 & 0 & 0 & 0 & 1 & 0 & 0 & 0 \\
0 & 0 & 0 & 0 & 0 & 0 & 0 & 1 & 0 & 0 \\
0 & 0 & 0 & 0 & 1 & 0 & 0 & 0 & 0 & 0 \\
0 & 0 & 0 & 0 & 0 & 0 & 0 & 0 & 1 & 0 \\
0 & 0 & 0 & 0 & 0 & 0 & 0 & 0 & 0 & 1
\end{array}\right)
$$


We can obtain $\mathbf{K}_{\text {sort }}$ using eq. (40)

$$
\mathbf{K}_{\text {sort }}=\left(\begin{array}{cc|ccccccccc}
\hline 0 & 0.26 & 0.06 & 0 & 0.05 & 0 & 0.06 & 0 & 0.2 & 0 \\
0 & 0 & 0.06 & 0.05 & 0 & 0 & 0.06 & 0 & 0.2 & 0 \\
\cline { 1 - 6 } 0 & 0 & 0 & 0.35 & 0.35 & 0.35 & 0 & 0.05 & 0 & 0 \\
0 & 0 & 0 & 0 & 0 & 0.13 & 0.35 & 0.05 & 0 & 0 \\
0 & 0 & 0 & 0 & 0 & 0.13 & 0.35 & 0.05 & 0 & 0 \\
0 & 0 & 0 & 0 & 0 & 0 & 0.35 & 0 & 0 & 0 \\
0 & 0 & 0 & 0 & 0 & 0 & 0 & 0.05 & 0 & 0 \\
0 & 0 & 0 & 0 & 0 & 0 & 0 & 0 & 0 & 0 \\
0 & 0 & 0 & 0 & 0 & 0 & 0 & 0 & 0 & 0 \\
0 & 0 & 0 & 0 & 0 & 0 & 0 & 0 & 0 & 0
\end{array}\right)
$$

The grey zones indicate the couplings among the nodes belonging to the same reduced node. When the system is condensed, these couplings will vanish, while the couplings outside the grey zones are those between the nodes belonging to different reduced nodes. These couplings will be summed up, to build the reduced conductive couplings matrix $\mathbf{K}^{\mathrm{R}}$. The same applies to the radiative couplings matrix. To compact the model (reduce the dimension of all vector and matrices defining the model), we need to construct the restriction matrix $\mathbf{P}$ (see 4.4.4)

$$
\mathbf{P}=\left(\begin{array}{lllll}
1 & 0 & 0 & 0 & 0 \\
1 & 0 & 0 & 0 & 0 \\
0 & 1 & 0 & 0 & 0 \\
0 & 1 & 0 & 0 & 0 \\
0 & 0 & 1 & 0 & 0 \\
0 & 1 & 0 & 0 & 0 \\
0 & 1 & 0 & 0 & 0 \\
0 & 1 & 0 & 0 & 0 \\
0 & 0 & 0 & 1 & 0 \\
0 & 0 & 0 & 0 & 1
\end{array}\right)
$$

Restriction matrix $\mathbf{P}$ allows us to reduce matrices $\mathbf{K}$ and $\mathbf{R}$

$$
\mathbf{K}^{\mathrm{R}}=\left(\begin{array}{ccccc}
0 & 0.327 & 0 & 0.4 & 0 \\
0 & 0 & 0.2 & 0 & 0 \\
0 & 0 & 0 & 0 & 0 \\
0 & 0 & 0 & 0 & 0 \\
0 & 0 & 0 & 0 & 0
\end{array}\right) \mathrm{W} / \mathrm{K} ; \quad \mathbf{R}^{\mathrm{R}}=\left(\begin{array}{ccccc}
0 & 4.4 & 0 & 0 & 4.5 \\
0 & 0 & 0 & 0 & 31.5 \\
0 & 0 & 0 & 0 & 0 \\
0 & 0 & 0 & 0 & 0 \\
0 & 0 & 0 & 0 & 0
\end{array}\right) \times 10^{-3} \mathrm{~m}^{2}
$$


and $\mathbf{C}, \mathbf{Q}$ and $\mathbf{T}$

$$
\mathbf{C}^{\mathrm{R}}=\left[\begin{array}{c}
25.9 \\
181.4 \\
100.0 \\
1000.0 \\
0
\end{array}\right] \mathrm{J} / \mathrm{K} ; \quad \mathbf{Q}^{\mathrm{R}}=\left[\begin{array}{c}
0 \\
0 \\
10.0 \\
0 \\
0
\end{array}\right] \mathrm{W} ; \quad \overline{\mathbf{T}}^{\mathrm{D}}=\left[\begin{array}{c}
49.7 \\
65.9 \\
116.1 \\
35.0 \\
50.0
\end{array}\right] \mathbf{C}
$$

Note that the vector of temperatures $\overline{\mathbf{T}}^{\mathrm{D}}$ corresponds to those derived directly from the detailed model, by calculating the thermal capacity-weighted mean temperatures. The reduced matrices and vectors $\mathbf{K}^{\mathrm{R}}, \mathbf{R}^{\mathrm{R}}, \mathbf{C}^{\mathrm{R}}$ and $\mathbf{Q}^{\mathrm{R}}$, together with the boundary conditions, make up a system that can be resolved -equation (4)-, to obtain the steady state solution of the reduced problem, $\hat{\mathbf{T}}^{\mathrm{R}}$ and $\hat{\mathbf{H}}^{\mathrm{R}}$

$$
\hat{\mathbf{T}}^{\mathrm{R}}=\left[\begin{array}{c}
49.7 \\
65.9 \\
115.9 \\
35.0 \\
50.0
\end{array}\right] \quad \mathrm{C} ; \quad \hat{\mathbf{H}}_{\mathrm{cb}}^{\mathrm{R}}=\left[\begin{array}{c}
5.88 \\
0
\end{array}\right] \mathrm{W} ; \quad \hat{\mathbf{H}}_{\mathrm{rb}}^{\mathrm{R}}=\left[\begin{array}{c}
0 \\
4.12
\end{array}\right] \mathrm{W}
$$

These temperatures and heat fluxes obtained by solving the reduced thermal model can be compared to those derived from the detailed model, to evaluate how accurate is the model condensation. The results of the correlation are shown in Table 5-1 and Table 5-2.

Table 5-1. 10-node model temperature results comparison.

\begin{tabular}{c||c|c|c|c|c}
$\begin{array}{c}i \\
\text { (condensed node) }\end{array}$ & 1 & 2 & 3 & 4 & 5 \\
\hline $\bar{T}_{i}^{D}\left({ }^{\circ} \mathrm{C}\right)$ & 49.7 & 65.9 & 116.1 & 35.0 & 50.0 \\
\hline$\hat{T}_{i}^{R}\left({ }^{\circ} \mathrm{C}\right)$ & 49.7 & 65.9 & 115.9 & 35.0 & 50.0 \\
\hline$\delta_{i}\left({ }^{\circ} \mathrm{C}\right)$ & $<0.1$ & $<0.1$ & 0.2 & $\begin{array}{c}\text { Boundary } \\
\text { node }\end{array}$ & $\begin{array}{c}\text { Boundary } \\
\text { node }\end{array}$
\end{tabular}

Table 5-2. 10-node model heat fluxes results comparison.

\begin{tabular}{c||c}
$\begin{array}{c}i \\
\text { (condensed node) }\end{array}$ & 4 \\
\hline$H_{c b_{i}}^{D}(\mathrm{~W})$ & 5.89 \\
\hline$\hat{H}_{c b_{i}}^{R}(\mathrm{~W})$ & 5.88 \\
\hline$\tilde{q}_{c b_{i}}$ & $0.17 \%$
\end{tabular}

\begin{tabular}{c||c}
$\begin{array}{c}i \\
\text { (condensed node) }\end{array}$ & 5 \\
\hline$H_{r b_{i}}^{D}(\mathrm{~W})$ & 4.11 \\
\hline$\hat{H}_{r b_{i}}^{R}(\mathrm{~W})$ & 4.12 \\
\hline$\tilde{q}_{r b_{i}}$ & $0.24 \%$
\end{tabular}

The temperatures and heat fluxes correlation, given by $\boldsymbol{\delta}, \tilde{\mathbf{q}}_{\mathbf{c b}}$ and $\tilde{\mathbf{q}}_{\mathbf{r b}}$, show that the model reduction is very good. 


\subsection{Solar Orbiter PHI Focal Plane Assembly test model}

In order to analyze the application of the method to a real problem with a large number of nodes, let us consider the thermal model of the Focal Plane Assembly (FPA) of the Polarimetric and Helioseismic Imager (PHI) instrument. PHI will be on board of the ESA mission Solar Orbiter, to be launched in 2020 [17, 72]. The FPA detailed thermal model consists of 1072 nodes and 457 geometric primitives, and has been entirely built with ESATAN-TMS [16]. The FPA is a camera system, with a detector, front-end electronics, and a cold finger, which connects the camera to the PHI Optics Unit cold element interface by means of a thermal strap. It also has an aluminum housing and an aluminum tube with some lenses. A render of the CAD model of the PHI FPA is shown in Fig. 5-4. The PHI FPA GMM is shown in Fig. 5-5. Fig. 5-6 shows the boundary nodes to which the model is conductively connected.

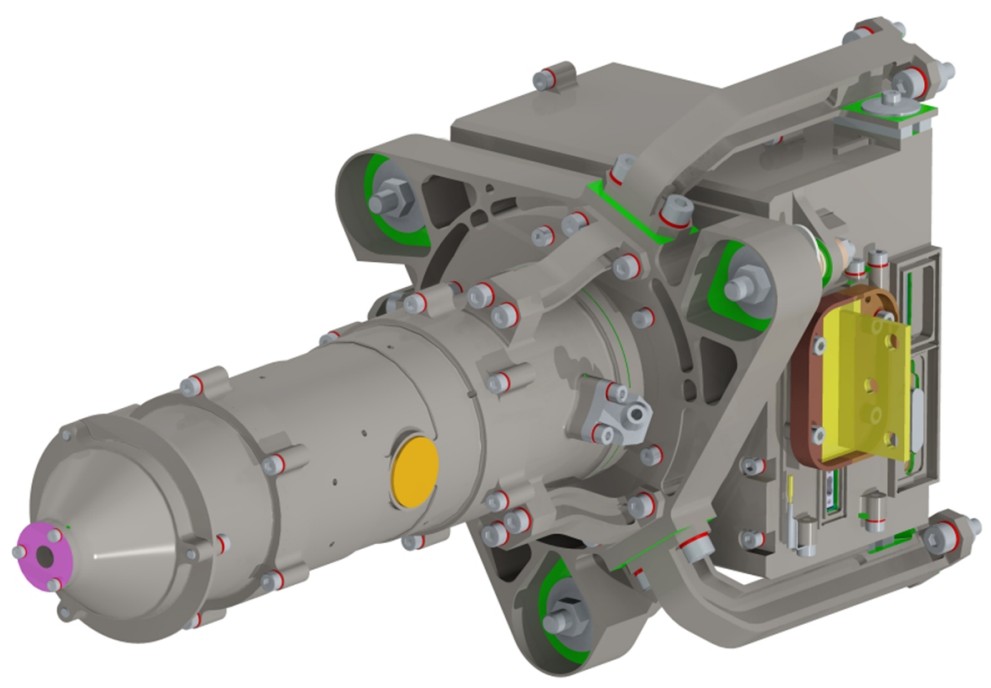

Fig. 5-4. PHI Optics Unit FPA CAD view.

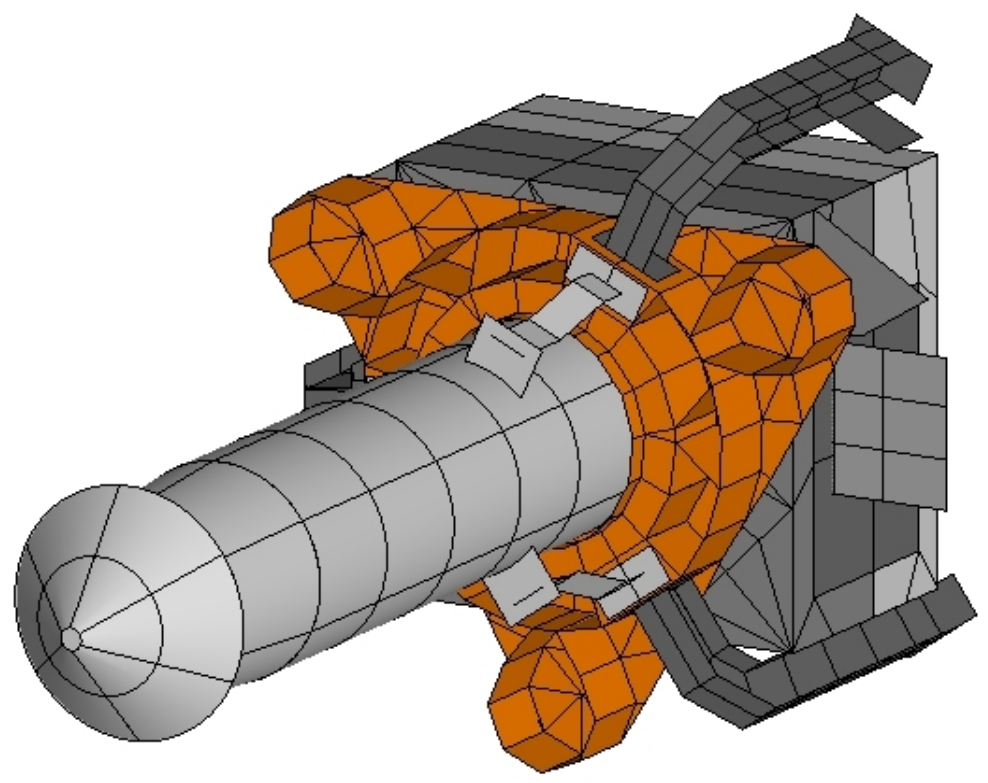

Fig. 5-5. PHI Optics Unit FPA GMM. 


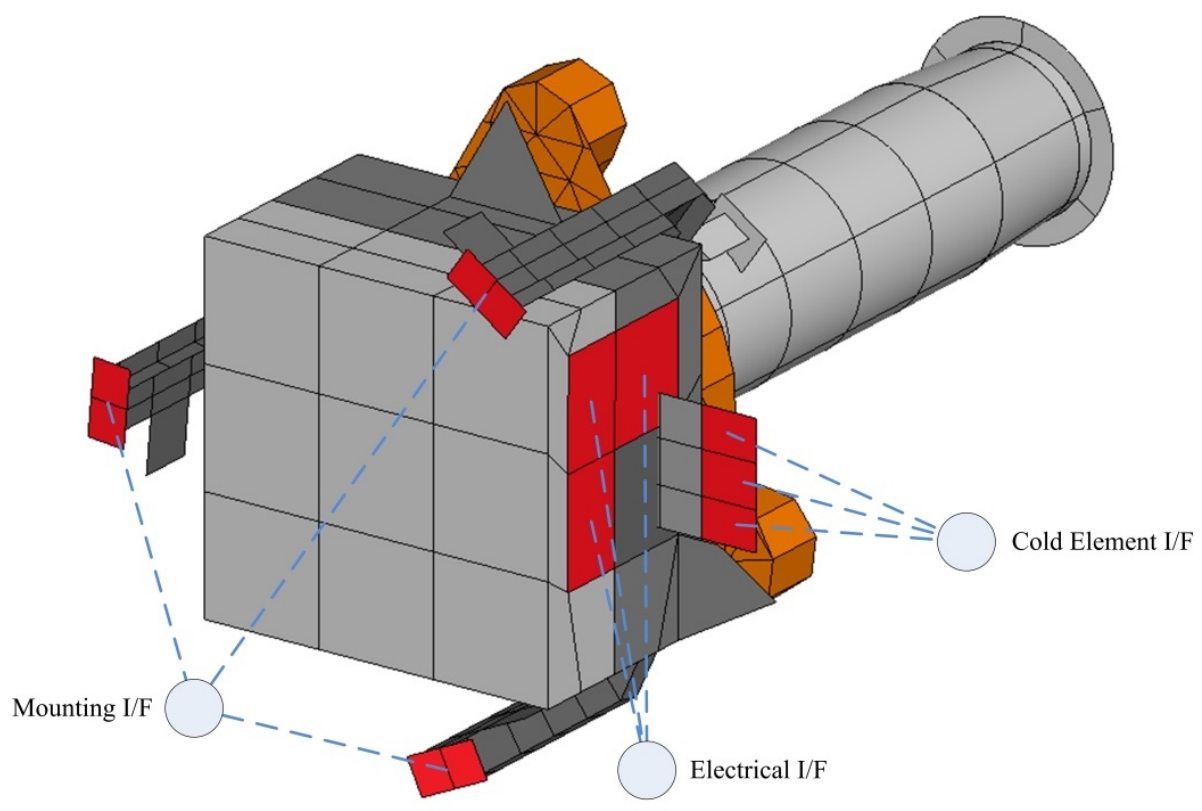

Fig. 5-6. PHI Optics Unit FPA boundary nodes (conductive).

\subsubsection{Reduction of the thermal mathematical model}

The conductive and radiative coupling matrices are shown in Fig. 5-7 as imported from the ESATAN-TMS TMD file. Matrix K has 2367 conductive couplings, whereas R has 299252. The matrix $\mathbf{R}$ is very dense not only because it represents the radiative couplings, but also due to the accuracy parameters used in the radiative calculations. The matrices are symmetric, so the program only works with upper triangular matrices. All the graphics related to the reduction process have been done with Matplotlib [73].

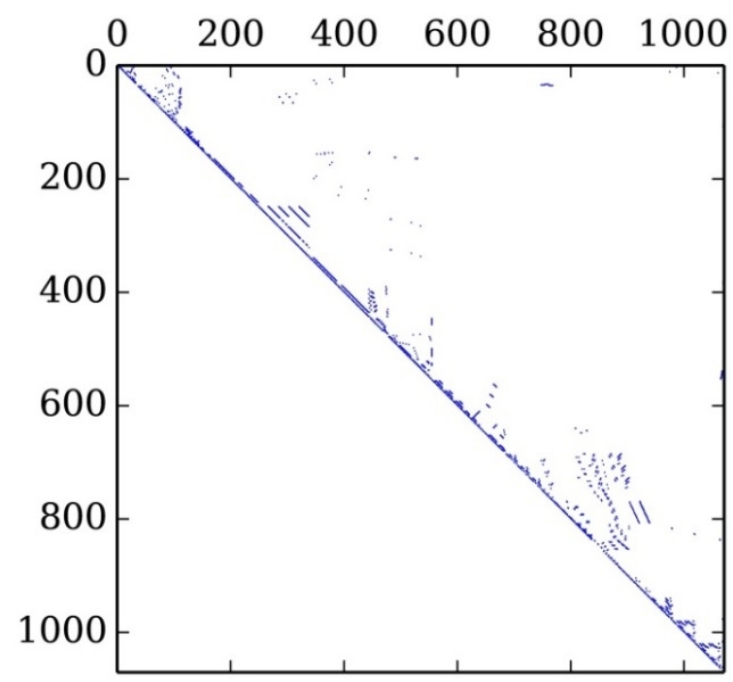

(a)

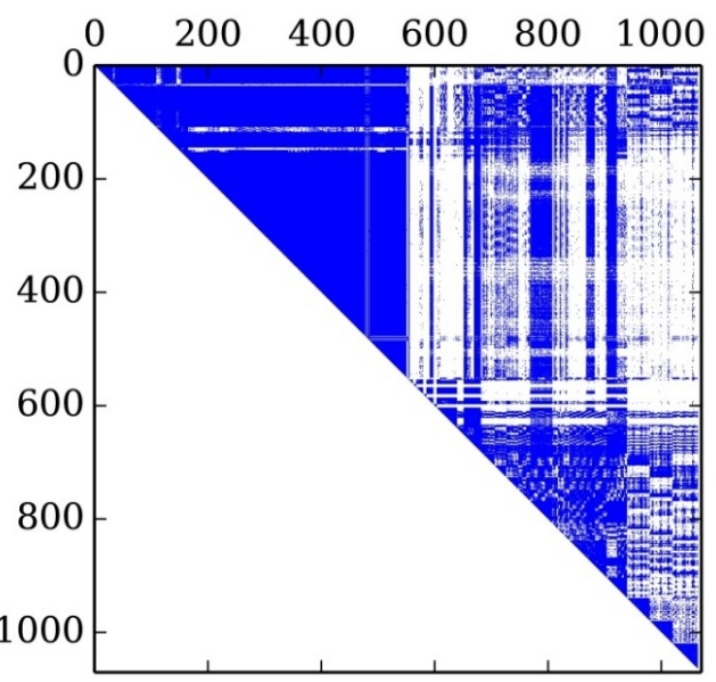

(b)

Fig. 5-7. Sparse graphical representation of (a) matrix $\mathrm{K}$ and (b) matrix $\mathrm{R}$. 
The model has four boundary nodes. If they are isolated, and the strongly connected components algorithm on the resulting matrix $\mathbf{K}^{\mathrm{B}}$ is run, the minimum number of condensed nodes is found to be 6 , including this number the 4 boundary nodes and 2 reduced diffusion nodes, one of which is conductively decoupled from the rest of the model. The maximum ratio $r_{r}$, that can be obtained according to eq. (23), is 0.998 (from 1072 to 6). This is a theoretical limit, but in order to be able to correlate the models according to typical correlation criteria, this value of $r_{r}$ will have to be lower. The final value of $r_{r}$ will depend on the final choice for $p_{f}$ and $\Delta T_{\max }$.

To prepare the matrix $\mathbf{K}$ for filtering, $\mu$ has been given the value calculated in section 4.2.1, $\mu=3.33 \cdot 10^{-5} \mathrm{~m}^{2} / \mathrm{s}$. Then, with $\mu$, together with the distance matrix $\mathbf{D}$ and the capacitance matrix $\mathbf{G}$, the sizing matrix $\mathbf{K}^{\mathrm{S}}$ can be obtained. The histogram of the conductive couplings matrices $\mathbf{K}$ and $\mathbf{K}^{\mathrm{S}}$ are shown in Fig. 5-8.

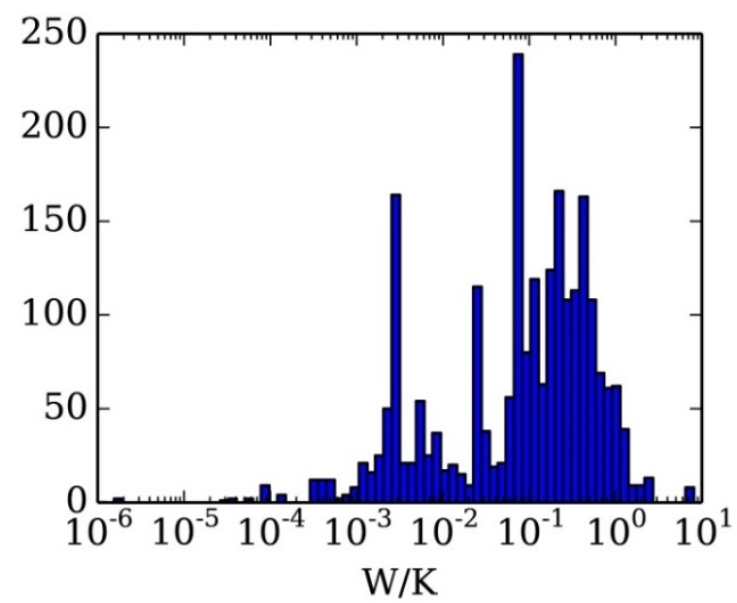

(a)

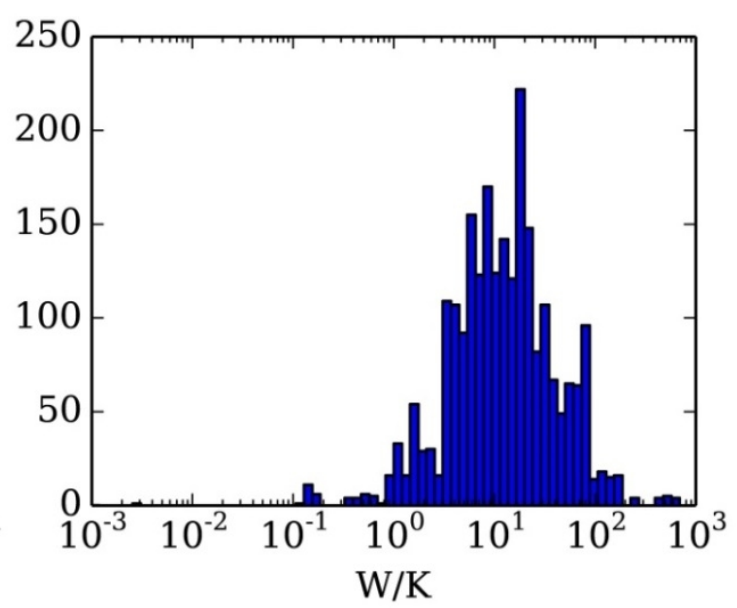

(b)

Fig. 5-8. Conductive couplings histograms of (a) matrix $K$ values and (b) matrix $K^{\mathrm{S}}$ values.

In Fig. 5-8, it can be seen that most of the couplings for matrix $\mathbf{K}$ are concentrated in the range $10^{-1}-10^{0} \mathrm{~W} / \mathrm{K}$, whereas for matrix $\mathbf{K}^{\mathrm{S}}$ they are mostly concentrated in the range $10^{1}-10^{2} \mathrm{~W} / \mathrm{K}$. This means that, according to the value chosen for parameter $\mu$ and the magnitudes used to estimate $\mathbf{K}^{\mathrm{S}}$, the model has a "poor" conductive behavior ( $\lambda$ has a generic value of $50 \mathrm{~W} /(\mathrm{m} \cdot \mathrm{K}))$. This appraisal would change if $\mu$ is changed, but it still gives an idea of how good (conductively) the model is, when compared with the estimator. If $\mu$ changes, the horizontal axis will change, but the shape of the histogram will remain.

Once the dimensionless coupling $\tilde{\mathbf{K}}^{\mathrm{I}}$ and temperature $\boldsymbol{\Theta}$ matrices have been created, it is possible to set up the adjacency matrix A, filter the system and compact the model. Before that, it was decided to do a preliminary study regarding how the reduction ratio $r_{r}$ varies with the different filtering levels. Fig. 5-9 shows the variation of $r_{r}$ with $p_{f}$ and $\Delta T_{\max }$ independently. This figure confirms what was explained in sections 4.2.3 and 4.3.1 relative to the behavior of $r_{r}$ as a function of $p_{f}$ and $\Delta T_{\max }$. 


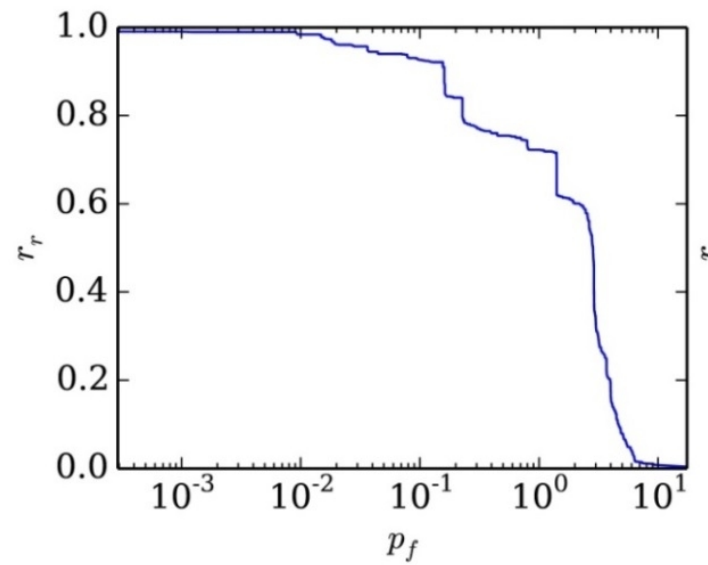

(a)

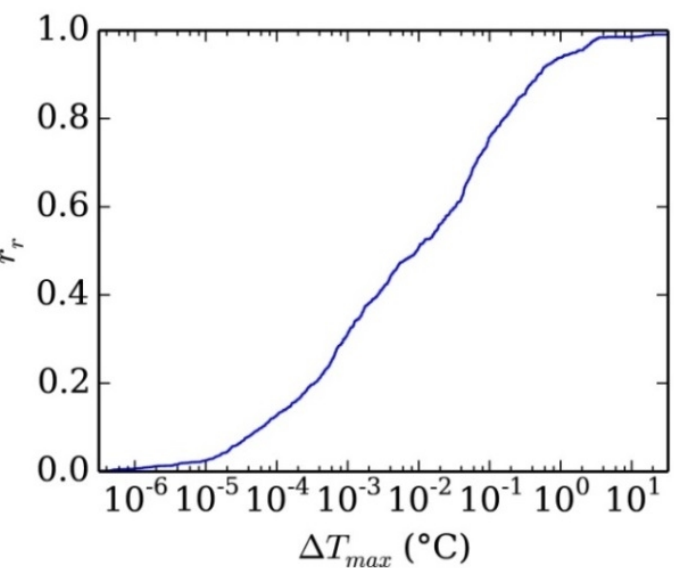

(b)

Fig. 5-9. Variation of (a) reduction ratio $r_{r}$ vs. filtering threshold $p_{f}$ and variation of (b) reduction ratio $r_{r}$ vs. temperature difference threshold $\Delta T_{\max }$. For (a), the SCC algorithm is run with $\tilde{\mathbf{K}}^{\mathrm{I}, \mathrm{F}}$. For (b), the matrix $\mathrm{K}$ is multiplied element-wise by $\Theta^{\mathrm{F}}$, and the SCC algorithm is run with the resulting matrix.

As the reduction ratio $r_{r}$ is a function of both $p_{f}$ and $\Delta T_{\max }$, to analyze the influence of these two parameters an appropriate method is to create a contour plot of the function $r_{r}=f\left(p_{f}, \Delta T_{\max }\right)$, as shown in Fig. 5-10. This way, a combination of $p_{f}$ and $\Delta T_{\max }$ suitable to get the desired value of $r_{r}$ can be selected. Furthermore, it is necessary to determine if the model correlation is good enough. To do this, correlation criteria for the reduced model should be defined.

For the sake of simplicity, only one is used for all the parts: $\delta_{\max }=3 \mathrm{~K}$. Regarding the heat flux exchanged with the boundary nodes, the chosen criteria are:

a. For absolute heat flow less than $q_{\text {lim }}=1 \mathrm{~W}$, difference must be less than $0.1 \mathrm{~W}$.

b. For absolute heat flow more than $q_{\text {lim }}=1 \mathrm{~W}$, difference must be less than $10 \%$.

Therefore, the value for $q_{\max }$ is $0.1 \mathrm{~W}$ and $\tilde{q}_{\max }$ is $10 \%$. Fig. 5-10 shows the function $r_{r}=f\left(p_{f}, \Delta T_{\max }\right)$, together with the correlation status for each pair of values $p_{f}$ and $\Delta T_{\max }$. The successful correlation implies that the three conditions (temperature difference, conductive and radiative heat fluxes differences) are satisfied. The values for $p_{f}$ and $\Delta T_{\max }$ cover almost the full range of both magnitudes, as they were shown in Fig. 5-9. The most extreme values have been omitted, since they produce almost no change in the reduction ratio $r_{r}$, as can be seen in Fig. 5-9. 


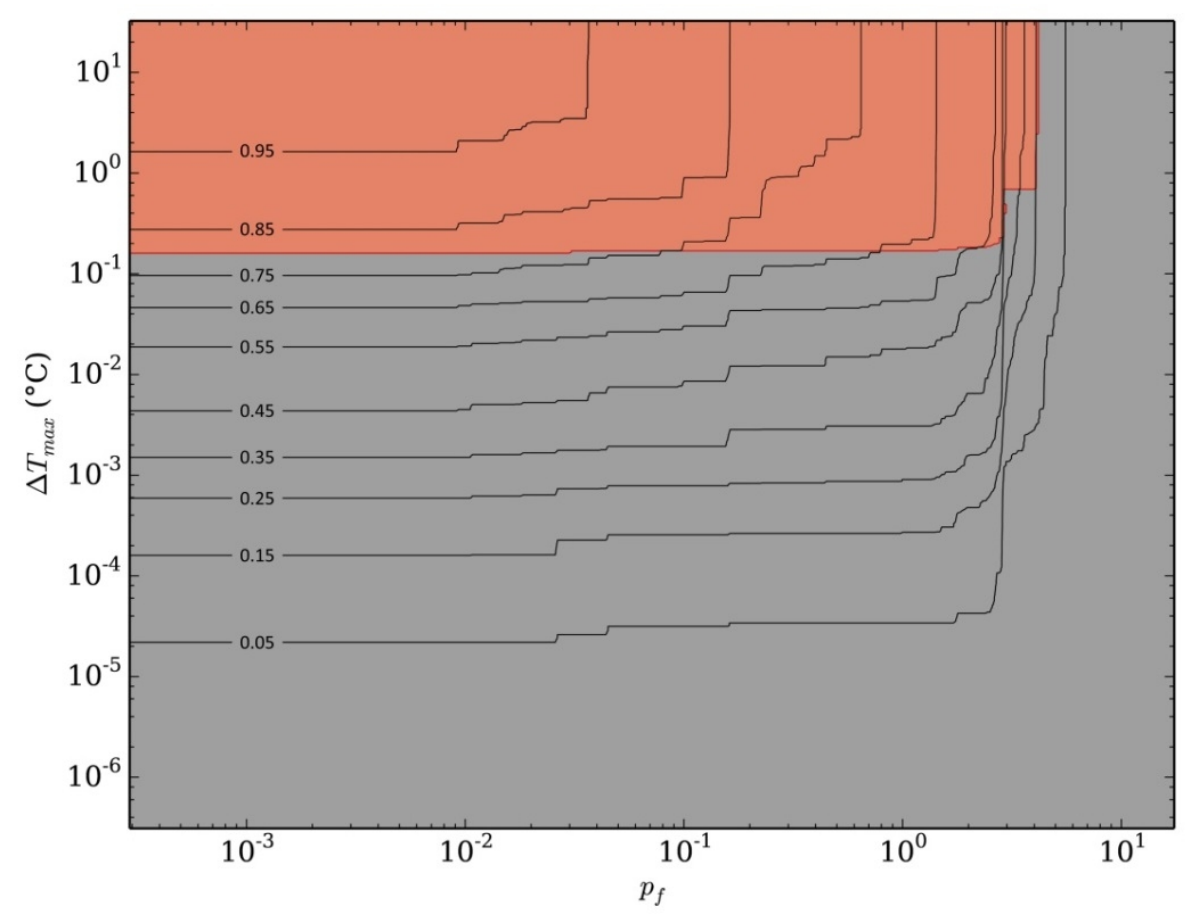

Fig. 5-10. Contour plot of the function $r_{r}=f\left(p_{f}, \Delta T_{\max }\right)$. Black solid lines represent constant $r_{r}$, whose value is indicated inline. Grey zone indicates successful correlation for the resulting reduced model. Orange colored zone indicates failed correlation for the resulting reduced model. Red line indicates the separation between both zones, being effectively the limit for a successful correlation.

Hence, it can be seen from the results shown in Fig. 5-10 that it is not possible to have a reduction ratio $r_{r}$ of more than $0.75-0.80$ that yields a successful correlation (with the current correlation criteria). This limitation is due to the PHI FPA physical characteristics, the correlation criteria and the reduction method itself. Other models could yield greater values of $r_{r}$ that satisfy the correlation criteria. In order to study in detail this model reduction in particular, let us plot the same Fig. 5-10 but zooming in on the range of $r_{r}=[0.50,0.90]$. This zooming in, and the displays of temperature and heat flux difference maps, are shown in Fig. 5-11 to Fig. 5-15. 


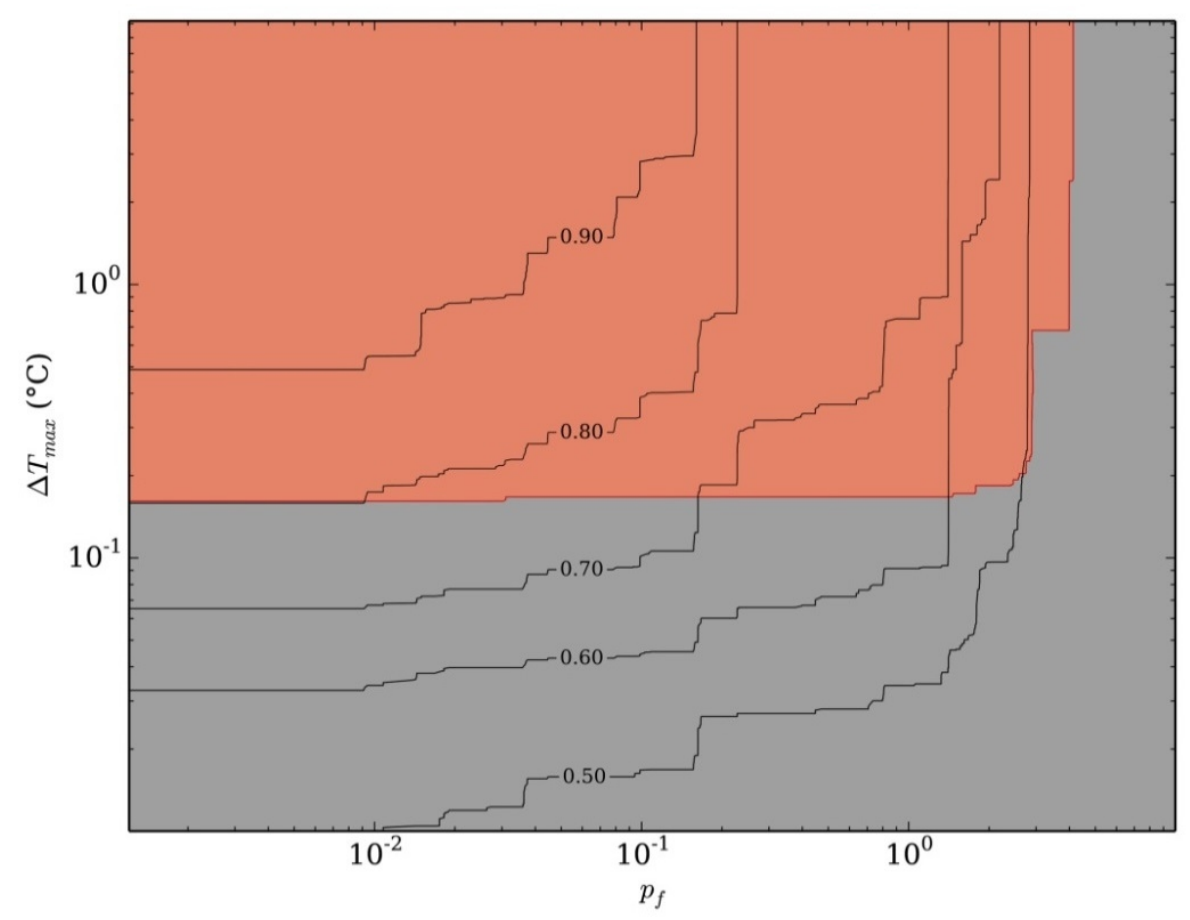

Fig. 5-11. Contour plot of the function $r_{r}=f\left(p_{f}, \Delta T_{\max }\right)$, zoomed at the $r_{r}=[0.5,0.9]$ zone.

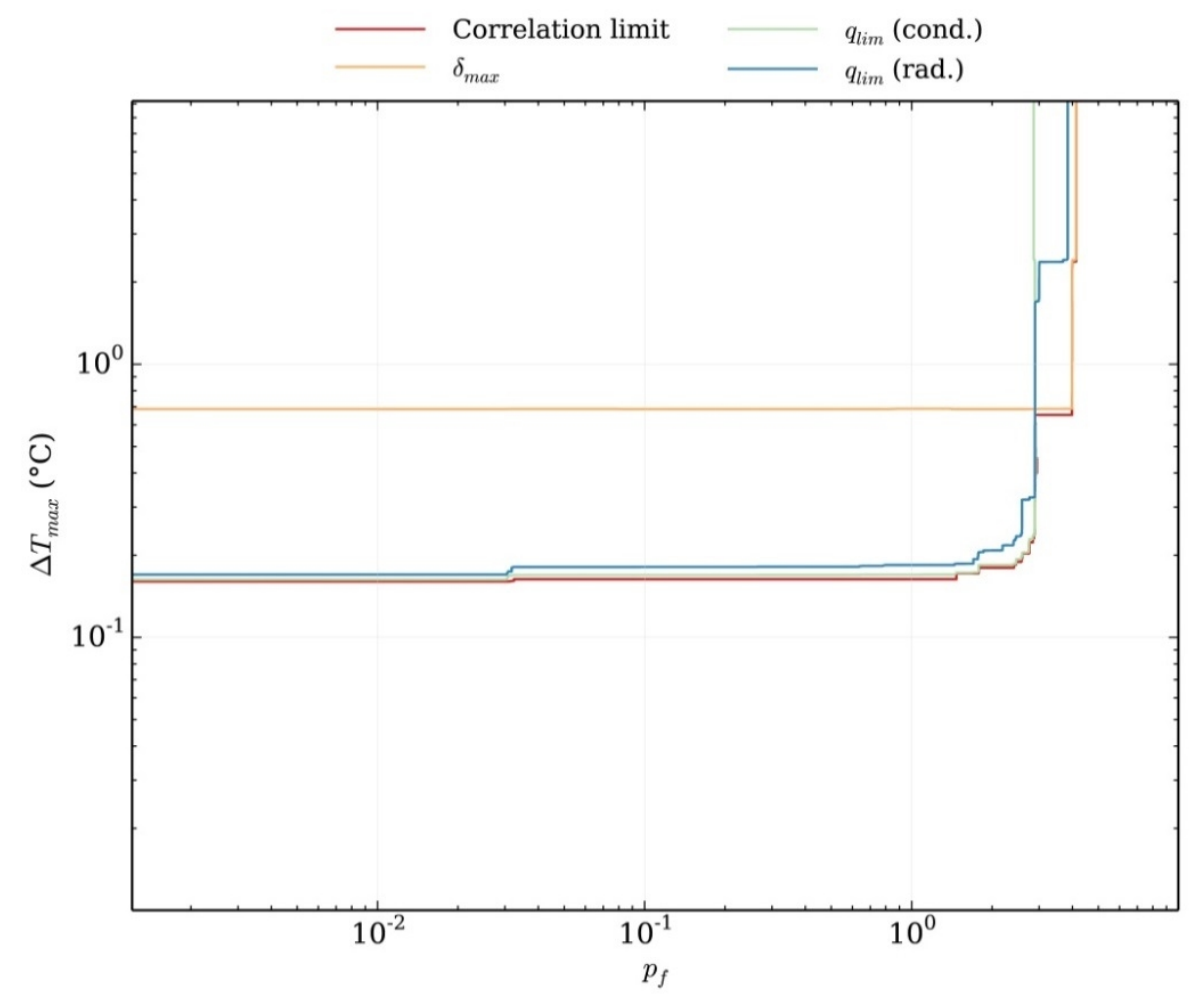

Fig. 5-12. Contour plot of the function $r_{r}=f\left(p_{f} \Delta T_{\max }\right)$, zoomed at the $r_{r}=[0.5,0.9]$ zone. Orange line indicates the correlation limit line based on $\delta$. Green line indicates the limit based on $q_{c b}$. Blue line is for $q_{r b}$. Red line is the envelope of the previous three, and coincides with the red line in Fig. 5-11. 


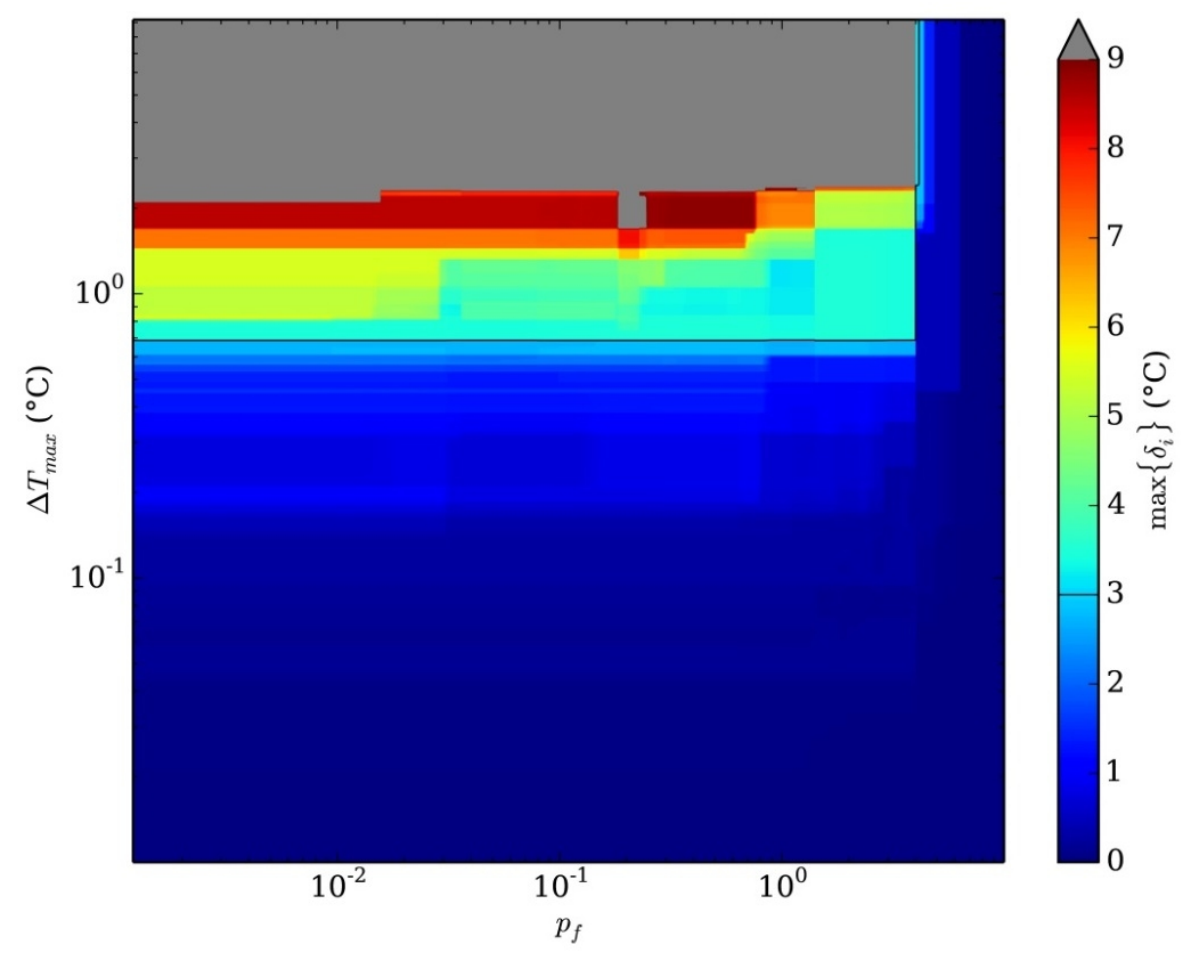

Fig. 5-13. $\operatorname{Max}\{\delta\}=f\left(p_{f} \Delta T_{\max }\right)$ over the zone $r_{r}=[0.5,0.9]$ zone. Black line: limit of the successful correlation zone in terms of temperature difference.

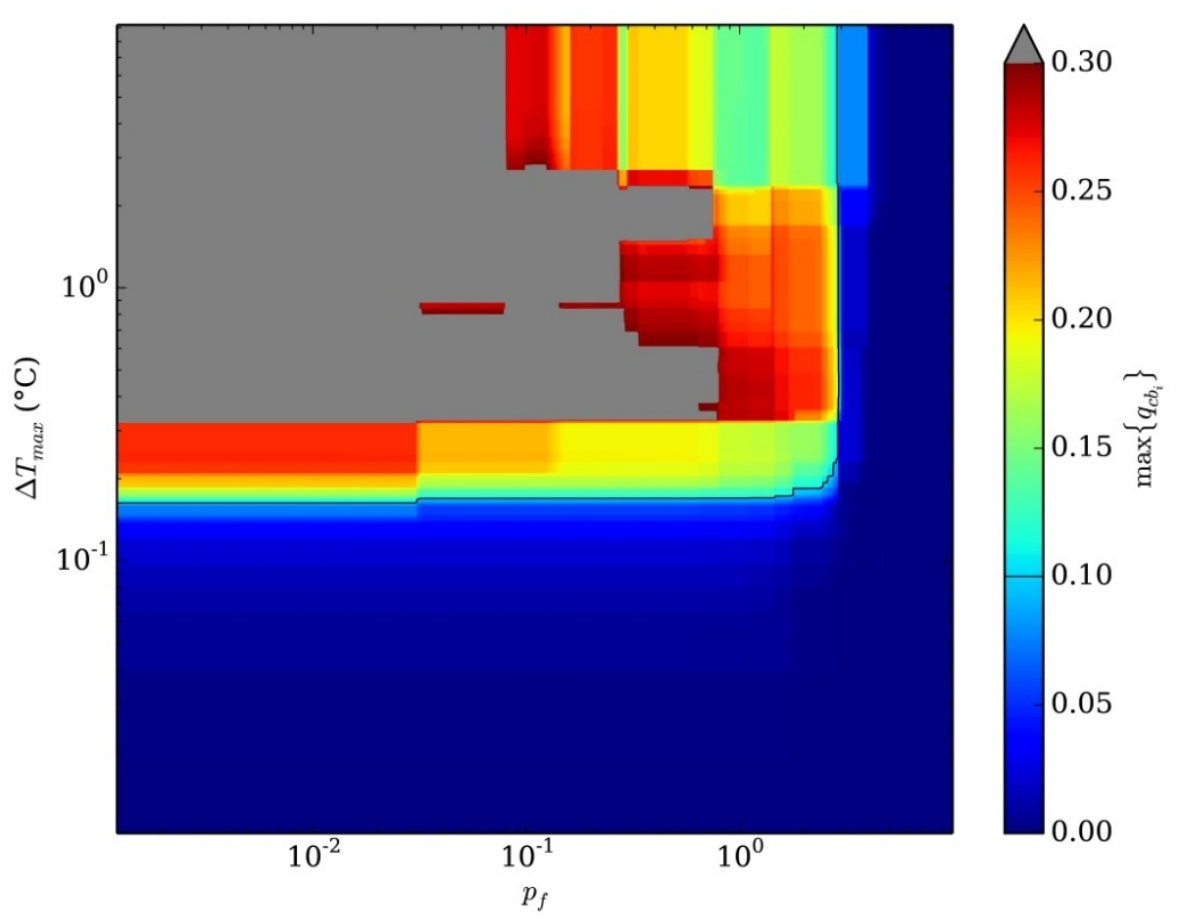

Fig. 5-14. $\operatorname{Max}\left\{q_{\mathrm{cb}}\right\}=f\left(p_{f}, \Delta T_{\max }\right)$ over the zone $r_{r}=[0.5,0.9]$ zone. Black line: limit of the successful correlation zone in terms of conductive heat flux difference. 


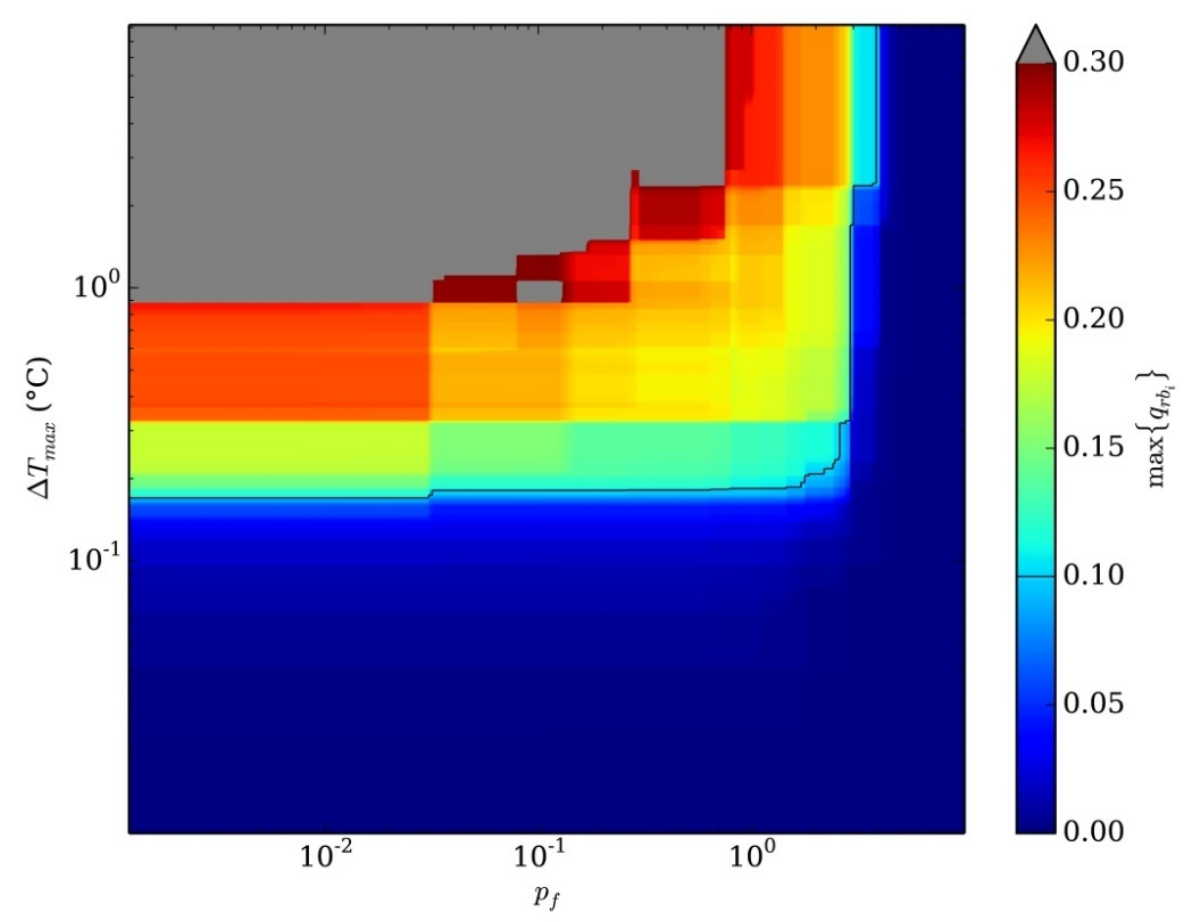

Fig. 5-15. $\operatorname{Max}\left\{q_{\mathrm{rb}}\right\}=f\left(p_{f}, \Delta T_{\max }\right)$ over the zone $r_{r}=[0.5,0.9]$ zone. Black line: limit of the successful correlation zone in terms of radiative heat flux difference.

As shown in Fig. 5-9, the variation of $r_{r}$ with $p_{f}$ is steep, whereas the variation of $r_{r}$ with $\Delta T_{\max }$ is more gradual. This behavior can be observed in Fig. 5-10. We can also see that the most restrictive criteria for the correlation are the heat flux differences (except for a small area where $p_{f} \approx 10^{1}$ and $\Delta T_{\max }$ ranges between $10^{\circ}$ and $10^{1}{ }^{\circ} \mathrm{C}$ ).

Let us select a combination of values for $p_{f}$ and $\Delta T_{\max }: p_{f}=0.001$ and $\Delta T_{\max }=0.16{ }^{\circ} \mathrm{C}$. After applying the SCC algorithm, these values yield $n_{c}=217$ nodes. With $n_{b}=4$, eq. (23) gives $r_{r}=0.80$. Other combinations of $p_{f}$ and $\Delta T_{\max }$ could have been picked, in case one had different criterion for convergence. The variation of $r_{r}$, maximum temperature difference and conductive and radiative heat flux differences through the boundaries are shown in Fig. 5-16, for a constant value of $\Delta T_{\max }$. This figure also shows that $\max \left\{\delta_{i}\right\}$ is not a monotonic decreasing function of $p_{f}$ The behavior of $\boldsymbol{\delta}, \mathbf{q}_{\mathbf{c b}}$ and $\mathbf{q}_{\mathbf{r b}}$ will depend of the particular structure of the model under study. 


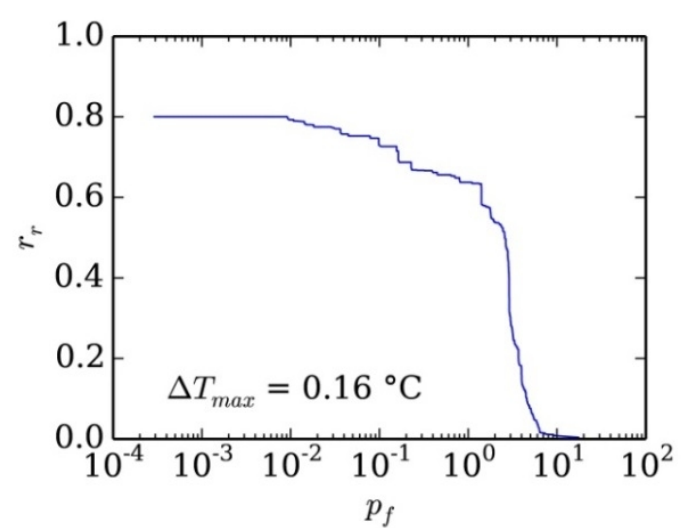

(a)

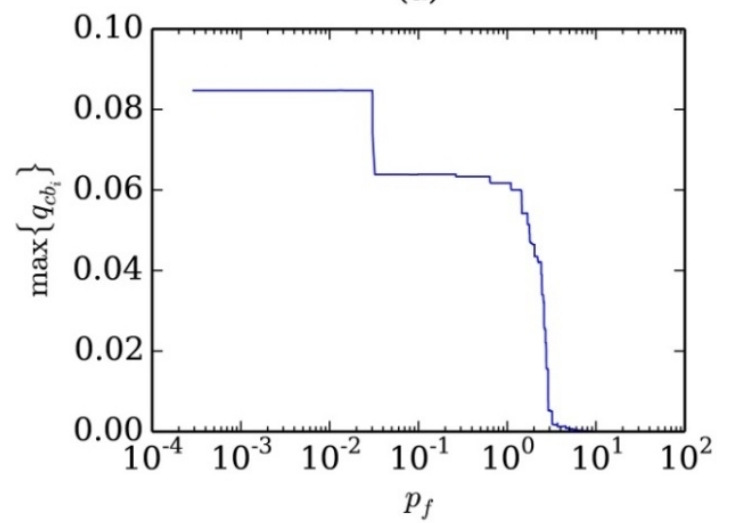

(c)

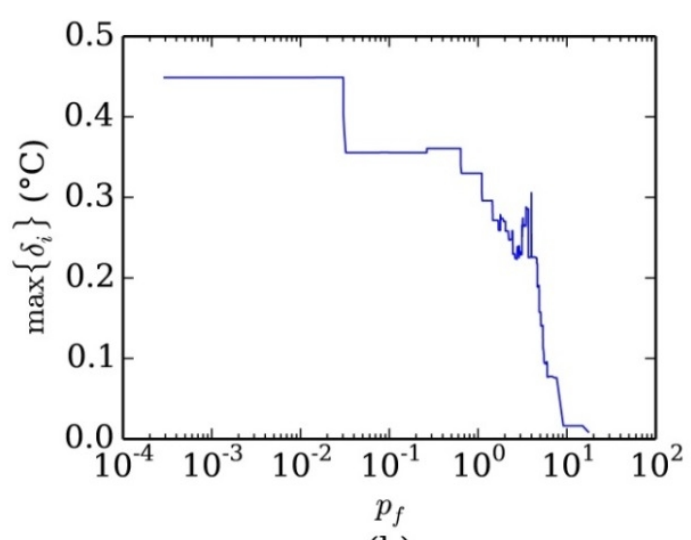

(b)

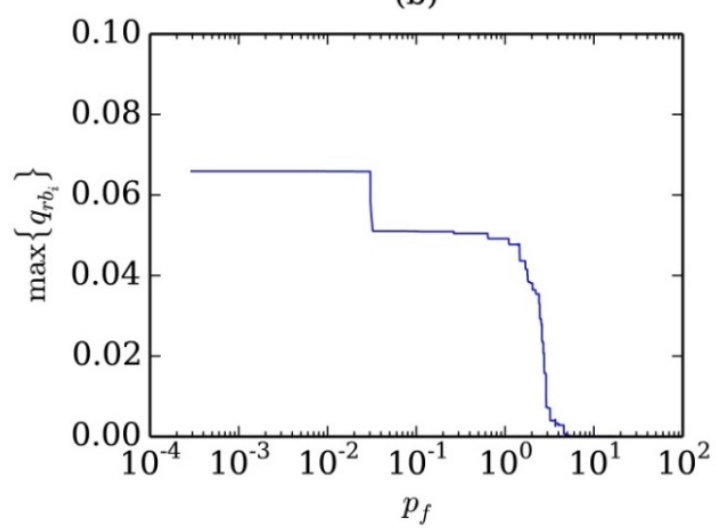

(d)

Fig. 5-16. (a) Variation of the reduction ratio $r_{r}$ as a function of filtering threshold $p_{f}$ keeping constant the temperature difference threshold $\Delta T_{\max }$ (b) is similar to (a), but showing $\max \left\{\delta_{i}\right\}$. (c) shows the variation of $\max \left\{q_{c b}\right\}$ as a function of $p_{f}$, again keeping constant $\Delta T_{\max }$. (d) represents $\max \left\{q_{r b}\right\}$.

The outcome of the SCC algorithm allows the restriction matrix $\mathbf{P}$ to be built (Fig. 5-17), as well as the sorted matrices $\mathbf{K}_{\text {sort }}$ and $\mathbf{R}_{\text {sort }}$ (Fig. 5-18). With the restriction matrix $\mathbf{P}$ the reduced matrices $\mathbf{K}^{\mathrm{R}}$ and $\mathbf{R}^{\mathrm{R}}$ can be calculated (Fig. 5-19). 


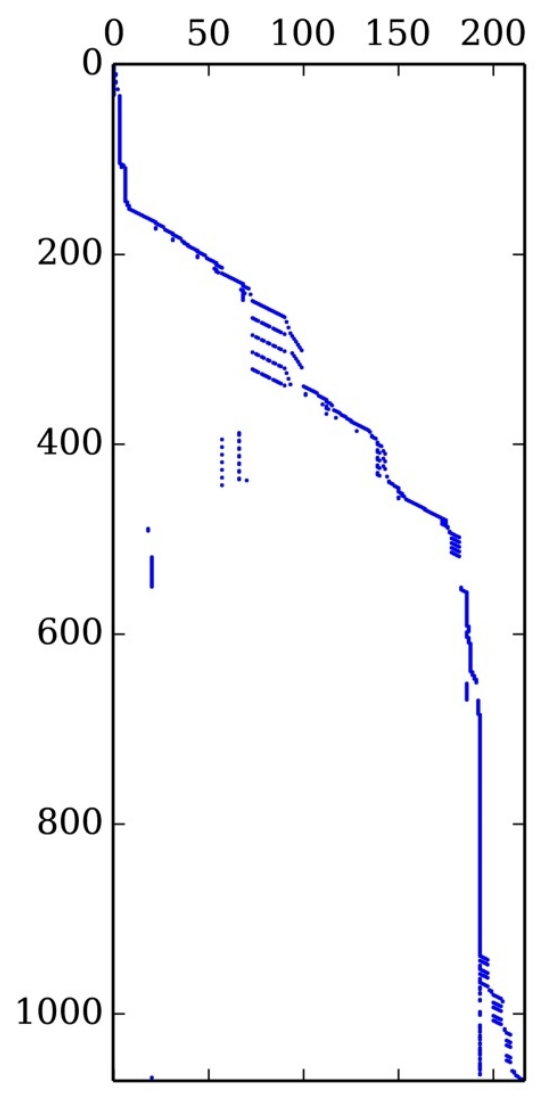

Fig. 5-17. Sparse graphical representation of restriction matrix $P$.

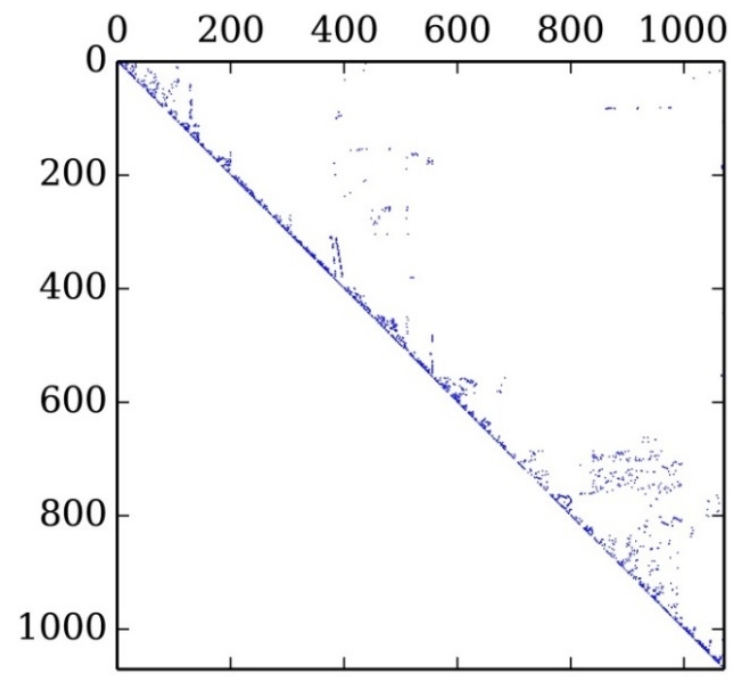

(a)

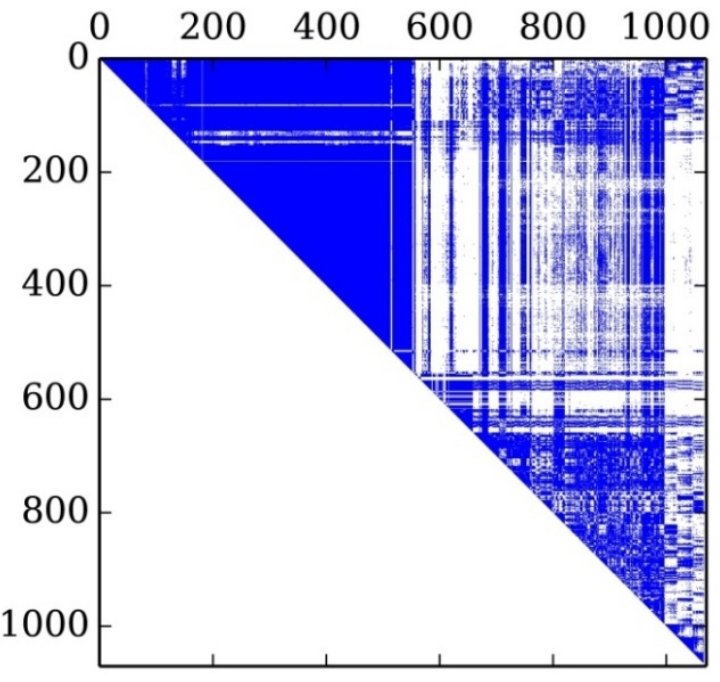

(b)

Fig. 5-18. Sparse graphical representation of (a) matrix $K_{\text {sort }}$ and (b) matrix $R_{\text {sort }}$ The matrices are symmetric, so the program only works with upper triangular matrices. 


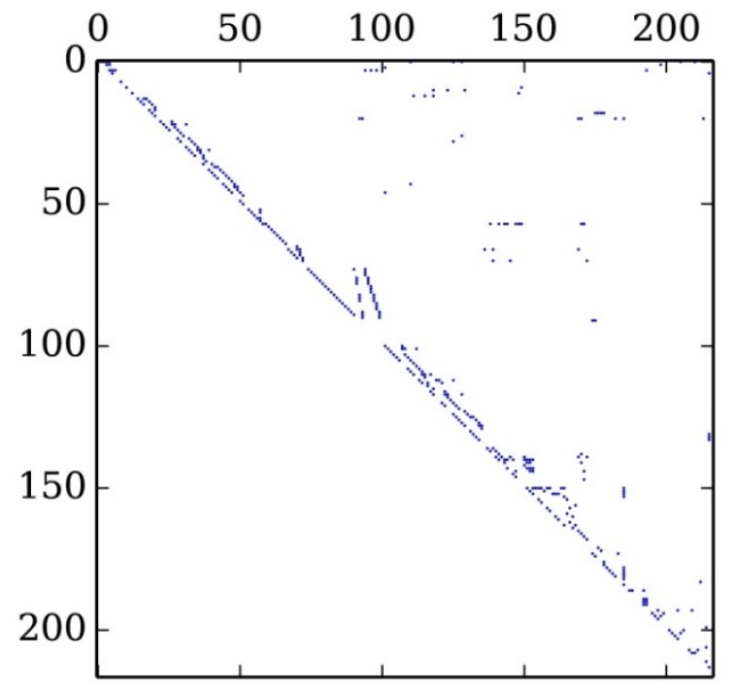

(a)

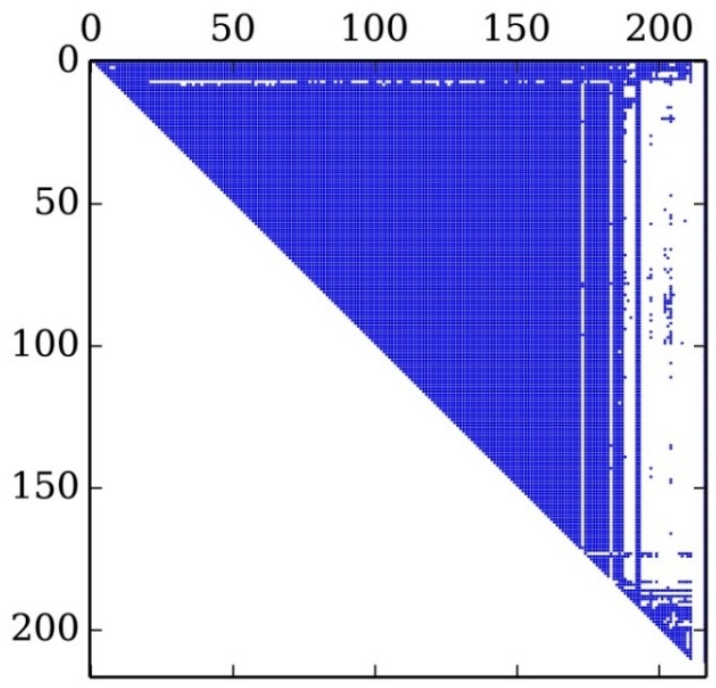

(b)

Fig. 5-19. Sparse graphical representation of (a) matrix $K^{R}$ and (b) matrix $R^{R}$. Matrix $K^{R} h a s$ 395 conductive couplings, whereas $R^{R}$ has 18 244. The matrices are symmetric, so the program only works with upper triangular matrices.

With the values of $p_{f}$ and $\Delta T_{\max }$ chosen, it is already known by looking at Fig. 5-11 that the correlation of the reduced model with the detailed model will be within the limits. The reduced system to find out the exact values for the temperature and heat fluxes differences can now be solved. Fig. 5-20 shows the components for the temperature difference vector $\boldsymbol{\delta}$. As can be deduced from this figure, the maximum value is around $0.35 \mathrm{~K}$, and the minimum is around -0.25 K. Fig. 5-21 shows the histograms of the values of vector $\boldsymbol{\delta}$ and vector $\boldsymbol{\tau}$ (see eq. (49)). 


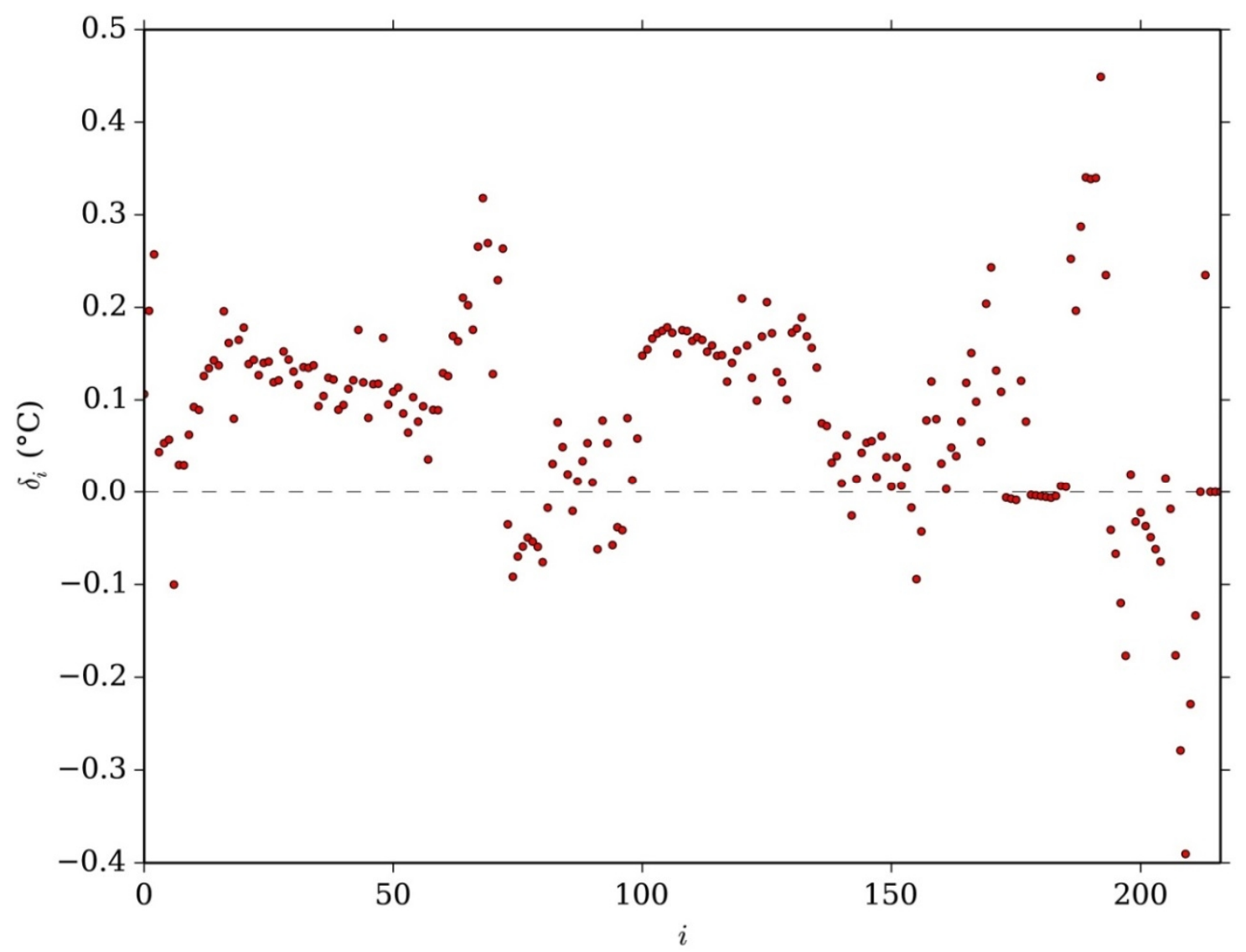

Fig. 5-20. Components of temperature differences vector $\delta$. $i$ : vector index.

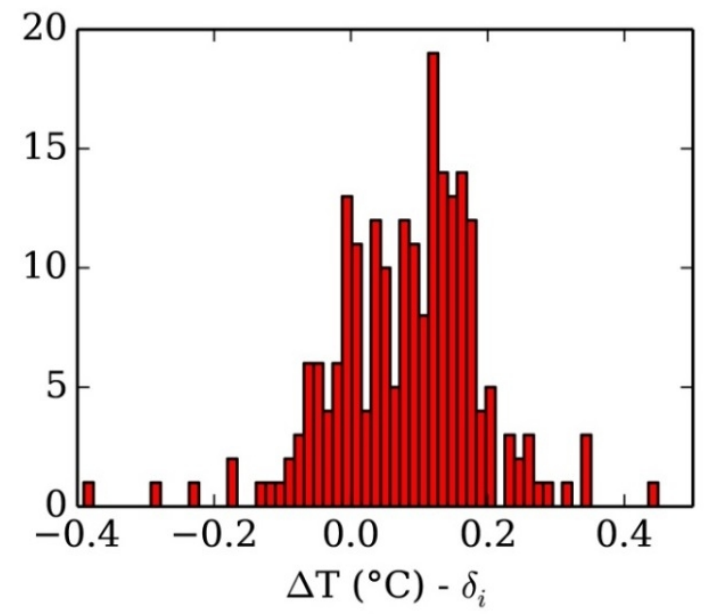

(a)

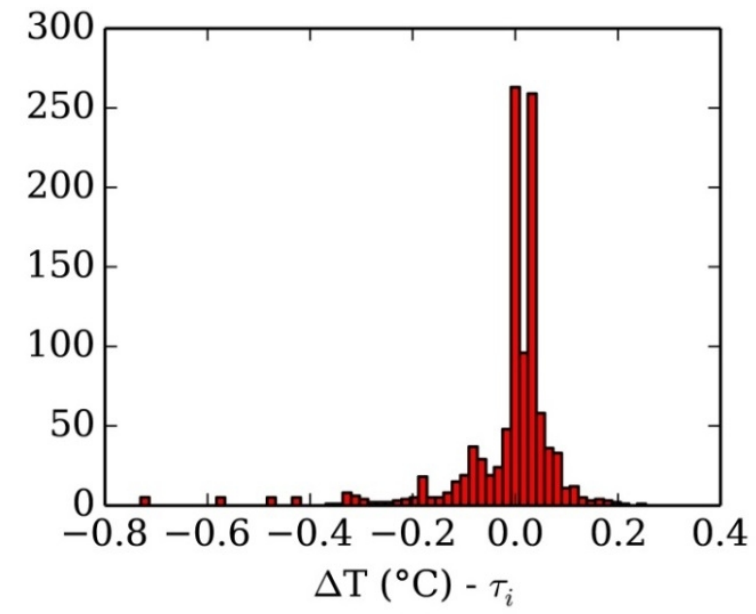

(b)

Fig. 5-21. Temperature differences histograms of (a) vector $\delta$ and (b) $\tau$.

Regarding the conductive heat flux differences, the values from the detailed model $\mathbf{H}_{\mathrm{cb}}^{\mathrm{D}}$ and those calculated with the reduced model $\hat{\mathbf{H}}_{\mathrm{cb}}^{\mathrm{R}}$ are shown in Table 5-3. 
Table 5-3. PHI FPA model conductive heat fluxes comparison.

\begin{tabular}{c||c|c|c|c|}
$\begin{array}{c}i \\
\text { (boundary node) }\end{array}$ & 1 & 2 & 3 & 4 \\
\hline$H_{c b_{i}}^{D}(\mathrm{~W})$ & 2.89 & 0.67 & 0.05 & - \\
\hline$\hat{H}_{c b_{i}}^{R}(\mathrm{~W})$ & 2.89 & 0.76 & 0.04 & - \\
\hline$q_{c b_{i}} / \tilde{q}_{c b_{i}}$ & $<0.1 \%$ & $<0.1 \mathrm{~W}$ & $<0.01 \mathrm{~W}$ & -
\end{tabular}

If both vectors are compared, it can be seen that the maximum difference occurs for the second boundary node. According to the definition given in eq. (50), together with our criteria about heat flux differences, it can be seen that the goal value is smaller than $1 \mathrm{~W}$, and consequently the difference must be given in absolute values (and not in percentage),

$$
q_{c b_{2}}=\left|H_{c b_{2}}^{D}-\hat{H}_{c b_{2}}^{R}\right|=0.09 \mathrm{~W}<q_{\max }=0.1 \mathrm{~W} .
$$

The radiative heat fluxes are shown in Table 5-4, where the difference, according to eq. (51) has been calculated as

$$
\tilde{q}_{r b_{4}}=\left|\frac{H_{r b_{4}}^{D}-\hat{H}_{r b_{4}}^{D}}{\min \left\{H_{r b_{4}}^{D}, \hat{H}_{r b_{4}}^{D}\right\}}\right|=6.8 \%<q_{\max }=10 \% .
$$

Table 5-4. PHI FPA model conductive heat fluxes comparison.

\begin{tabular}{c||c|c|c|c|}
$\begin{array}{c}i \\
\text { (boundary node) }\end{array}$ & 1 & 2 & 3 & 4 \\
\hline$H_{r b_{i}}^{D}(\mathrm{~W})$ & - & - & - & -1.17 \\
\hline$\hat{H}_{r b_{i}}^{R}(\mathrm{~W})$ & - & - & - & -1.25 \\
\hline$q_{r b_{i}} / \tilde{q}_{r b_{i}}$ & - & - & - & $<6.8 \%$
\end{tabular}

The program takes 30 hours in a mid-2014 desktop computer to run 293046 cases (reduced models obtained with different combinations of $p_{f}$ and $\Delta T_{\max }$ ), in order to plot Fig. 5-10 to Fig. 5-15. This time can be reduced by modifying the sampling space; although the figures would lose some resolution, it would be still enough for the sake of assessing the combination of parameters. 


\subsubsection{Reduction of the geometrical mathematical model}

Based on the reduction scheme developed in section 5.2.1, it is necessary to reduce the GMM accordingly. Although it would be possible to develop a kind of automatic algorithm to reduce automatically the GMM [32], the manual reduction serves its purpose, and it is not as time consuming as the TMM reduction. The program produces a text report with all the statistics of the condensation (an extract of this report can be found in section 8.2). This information, in its full extension, also includes what detailed model nodes belong to each single reduced node, with their original temperatures, labels, etc. In addition to that, the code generates a TMD file suitable for ESATAN-TMS. The TMD file allows the user to see the condensed nodes number and temperatures overlaid on the GMM, the original detailed model nodes, and the temperature difference between detailed and condensed nodes - components of vector $\tau$, see eq. (49)-. A couple of snapshots of the post-processed TMD file temperature differences are shown in Fig. 5-22.
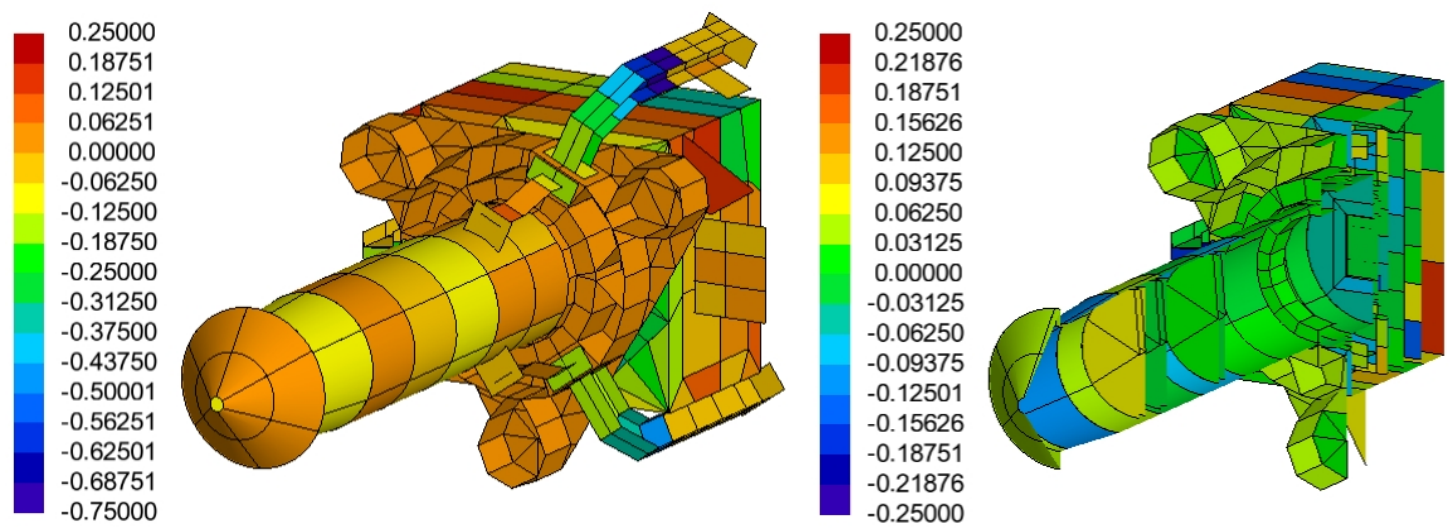

Fig. 5-22. Temperature differences $\left({ }^{\circ} \mathrm{C}\right)$ between detailed and reduced nodes $(\tau)$ overlaid on the GMM.

Regarding the geometrical arrangement, some of the nodes from the detailed model are condensed in a very unpleasant way (in what respects the geometrical reduction). For instance, the reduced nodes in the FPGA board are depicted in Fig. 5-23. The board was modelled in the detailed GMM as a single shell, with a mesh of $7 \times 7$ nodes. In the reduced model, the board is divided in 36 nodes. In order to represent accurately the reduced nodes in the reduced GMM, one would have to create dedicated shells for each one of the reduced nodes, producing a kind of "patchwork". This could be very time consuming, but the geometry would adapt completely to the mesh created by the reduction algorithm. 


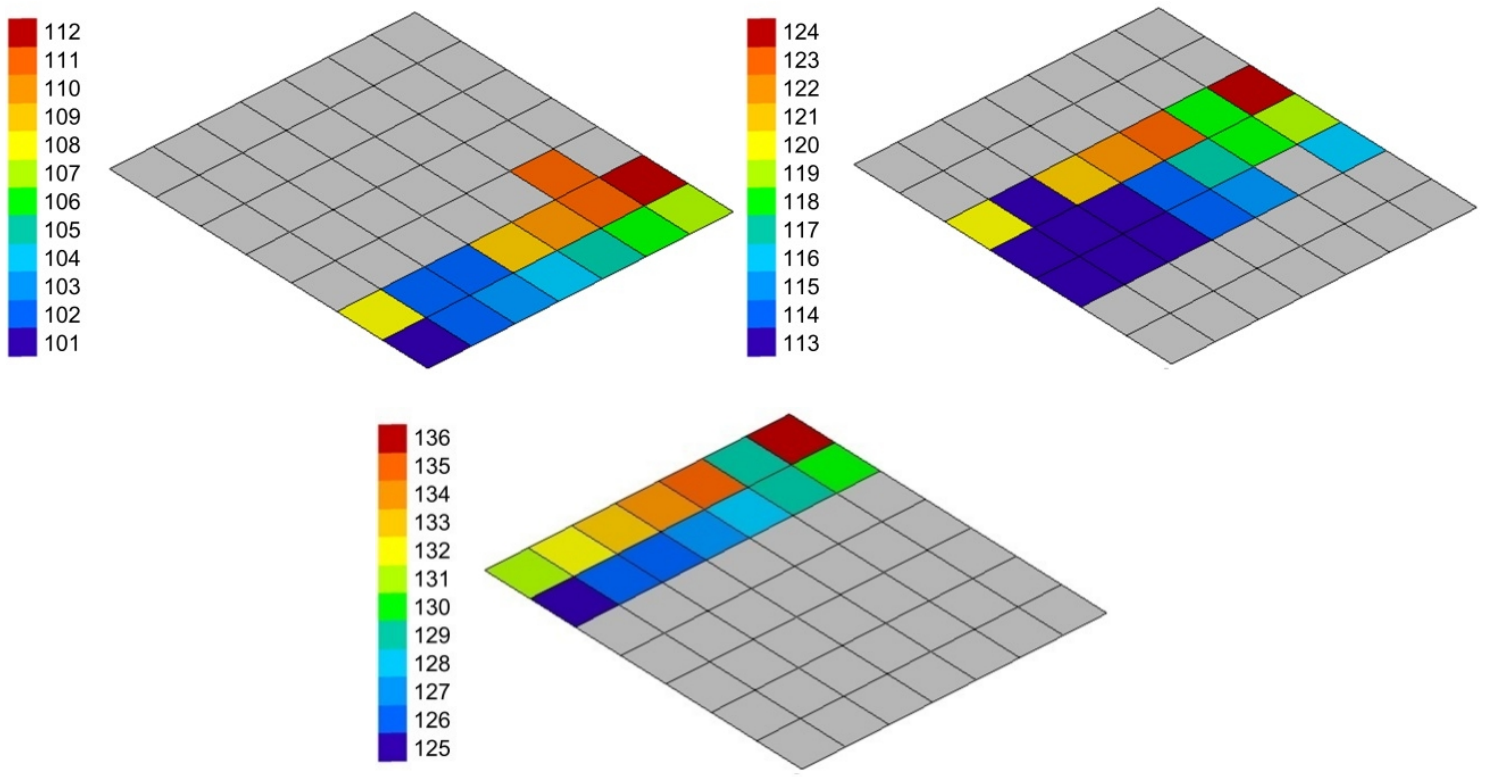

Fig. 5-23. Condensed node number overlaid on the FPGA GMM (top left for node range 101112, top right for 113-124 and bottom for 125-136).

Overall, depending on the TMM reduction achieved, the reconstructed RGMM might pose no significant saving in terms of radiative calculations time. Although the number of nodes is reduced, it might be necessary to use many shells in the RGMM in order to adapt the geometry to the reduced nodes, which increases the computation time for calculating the REFs. 


\subsubsection{Transient analysis}

The transient correlation is beyond the scope of this study, but a simple transient case has been run in ESATAN-TMS in order to compare the results obtained with both detailed and reduced models. The evolution of the temperatures has been calculated for a total time of $16200 \mathrm{~s}$. The model is brought from hot to cold conditions, while the power dissipation values remain constant. The variation of the boundary nodes temperatures with time used for the transient analysis is shown in Fig. 5-24, and is given by

$$
\left\{\begin{aligned}
T_{C E \mathrm{IF}}(t) & =\left\{\begin{array}{rr}
\left(-\frac{t}{180}-25\right) \mathrm{C}, & 0<t \leq 1800 \mathrm{~s}, \\
-35 \mathrm{C}, & t>1800 \mathrm{s.}
\end{array}\right. \\
T_{F B \text { cond I/F }}(t) & =\left\{\begin{array}{rr}
\left(55-\frac{t}{40}\right) \mathrm{C}, & 0<t \leq 1800 \mathrm{~s}, \\
10 \mathrm{C}, & t>1800 \mathrm{~s} .
\end{array}\right. \\
T_{\text {Elec IF }}(t) & =\left\{\begin{array}{rr}
\left(55-\frac{t}{45}\right) \mathrm{C}, & 0<t \leq 1800 \mathrm{~s}, \\
15 \mathrm{C}, & t>1800 \mathrm{~s} .
\end{array}\right. \\
T_{\text {Rad env }}(t) & =\left\{\begin{array}{rr}
\left(60-\frac{t}{30}\right) \mathrm{C}, & 0<t \leq 1800 \mathrm{~s}, \\
0 \mathrm{C}, & t>1800 \mathrm{s.} .
\end{array}\right.
\end{aligned}\right.
$$

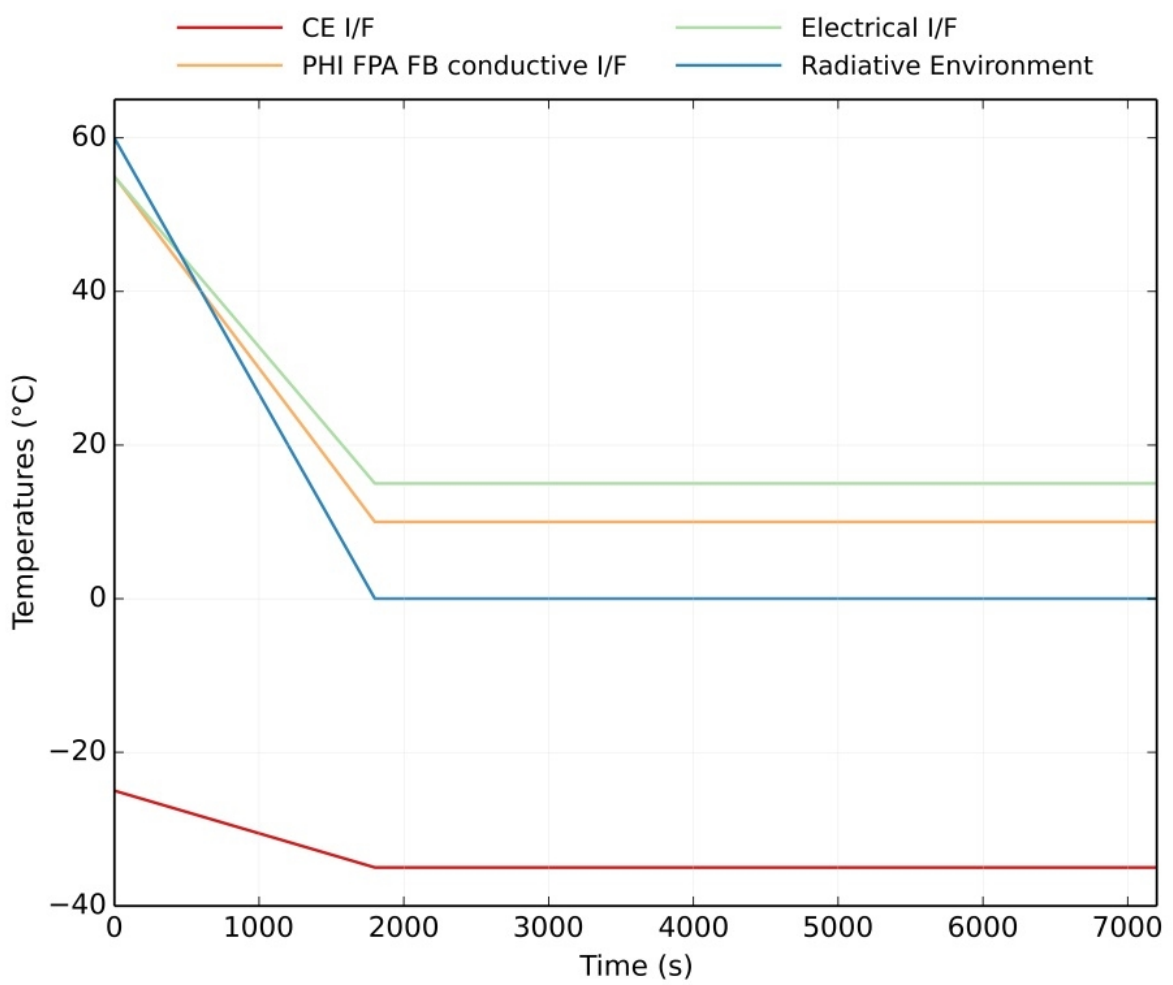

Fig. 5-24. Evolution of the boundary nodes temperatures for transient analysis (horizontal axis -time- only covers just up to $7200 \mathrm{~s}$ for more clarity). 
The ESATAN control constant values used in the analyses can be found in Table 5-5.

Table 5-5. ESATAN control constants values.

\begin{tabular}{c||c|l|} 
Control constant & Value & Definition \\
\hline DTIMEI & $0.025 \mathrm{~s}$ & Input time step \\
\hline OUTINT & $60 \mathrm{~s}$ & Output time step \\
\hline RELXCA & $10^{-4}{ }^{\circ} \mathrm{C}$ & Temperature convergence criterion \\
\hline NLOOP & 500 & Maximum allowable number of iterations
\end{tabular}

The input time step DTIMEI value has been chosen following the guidelines given in [69]. These guidelines advise the use of a DTIMEI value smaller than the CSGMIN value for the model under analysis ${ }^{10}$. The term CSG stands for the ratio of a node thermal capacitance divided by the sum of the conductances to the node. This number measures "the rate at which the node temperature responds to a given heat impulse" [16], and CSGMIN is the minimum value of CSG for a given model. For the case of the reduced model of the PHI OPT FPA, CSGMIN $=0.293 \mathrm{~s}$ at node 182 (which represents a group of five nodes belonging to the cold finger) and for the detailed model, CSGMIN $=0.03 \mathrm{~s}$ at node 13520 (thermal baffle sealing). Although the models have different CSGMIN, it has been used the same DTIMEI value (the most restrictive one). The transient analysis has taken roughly 4.5 minutes for the reduced model and 100 minutes for the detailed one, both run in a 2014 laptop (Intel® Core i5-4210U, maximum frequency (turbo) of $2 \times 2.7 \mathrm{GHz}$ ). This gives an idea of the computational time saving that can be achieved by reducing the model and using it for transient calculations.

The temperatures obtained in the DTMM transient analysis have been surface-weighted averaged following the same approach indicated in eq. (43). Results show that most of the elements are also well correlated ${ }^{11}$ in the transient analysis. The maximum temperature differences occur for the condensed nodes no. $190\left(T_{\text {DTMM }}-T_{R T M M}=3.8{ }^{\circ} \mathrm{C}\right.$, part of the lens tube) and no. $210\left(T_{D T M M}-T_{R T M M}=-5.4{ }^{\circ} \mathrm{C}\right.$, one of the mounting legs $)$. The nodes are shown in Fig. 5-26. Both temperature differences evolution can be seen in Fig. 5-25. The temperature differences evolution for other nodes are shown in Fig. 5-27, and their positions in the GMM are shown in Fig. 5-28. Of course, the correlation depends strongly on the rate of change of the boundary conditions (in the sense that diverging transient temperatures amplify the errors made in the steady state reduction). For this analysis, the variation profiles of the boundary conditions have been defined using a unique time basis, and the maximum rate of change of $2{ }^{\circ} \mathrm{C} / \mathrm{min}$

\footnotetext{
${ }^{10}$ Other authors, such as [86], questioned the necessity of using such a small input time step.

${ }^{11}$ The TMM correlation success criteria in transient conditions are not as standardized as those used for steady state analysis. As [87] indicates in section 4.5.3.3, the transient modes correlation needs specific success criteria to be defined. This statement is formulated for test-model correlation, but [69] suggests that the test-model correlation criteria "can be used as inspiration to derive guidelines for model reduction".
} 
happens for the radiative environment. This value is the maximum rate of change expected during a PHI OPT FPA thermal vacuum test, and it is faster than the variation predicted in flight. If it were necessary to achieve a better level of temperatures correlation, it might help to use more reduction snapshots along the transient trajectory, but this has not been tested. In theory it would also be possible to slightly adjust the thermal capacitances in the reduced model, always keeping the total sum identical in both detailed and reduced models, for instance, using TAUMEL, an automated correlation tool developed by Frey, Trinoga et al [74, 75], or the method proposed by Anglada and Garmendia [76], based on genetic algorithms. Theoretically, this adjustment might have an impact in the steady state correlation, since changing the thermal capacities would affect the way in which the model is reduced (32) and how the temperatures are condensed from the detailed model (43) (in principle, it would not be necessary to recalculate the detailed model condensed temperatures and to reduce again the model). In addition, the conductive couplings could be slightly adjusted in order to improve the transient correlation. Again, this process should be done carefully, although it is beyond the scope of this study.

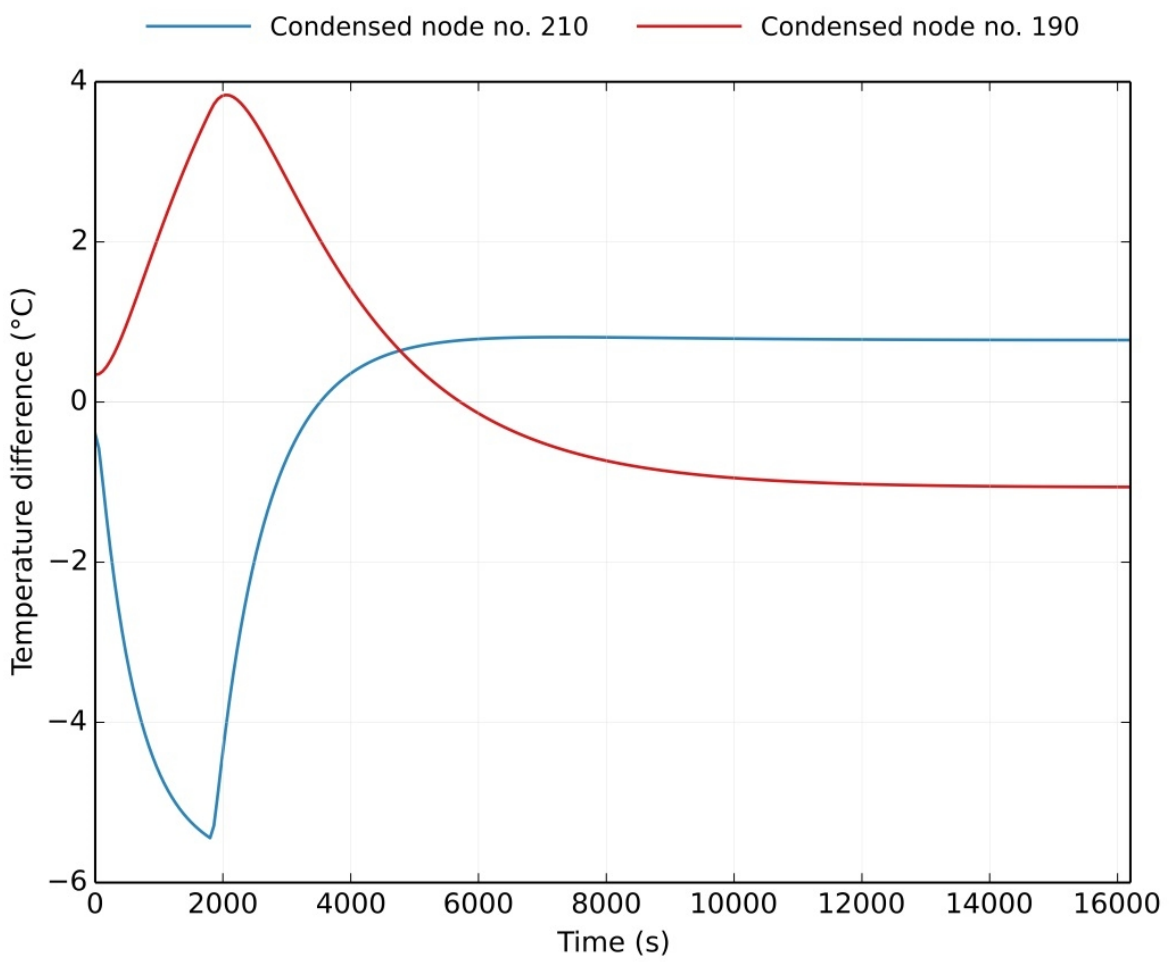

Fig. 5-25. Evolution of the temperatures of nodes 190 and 210 (corresponding to maximum differences obtained in the transient analysis). 

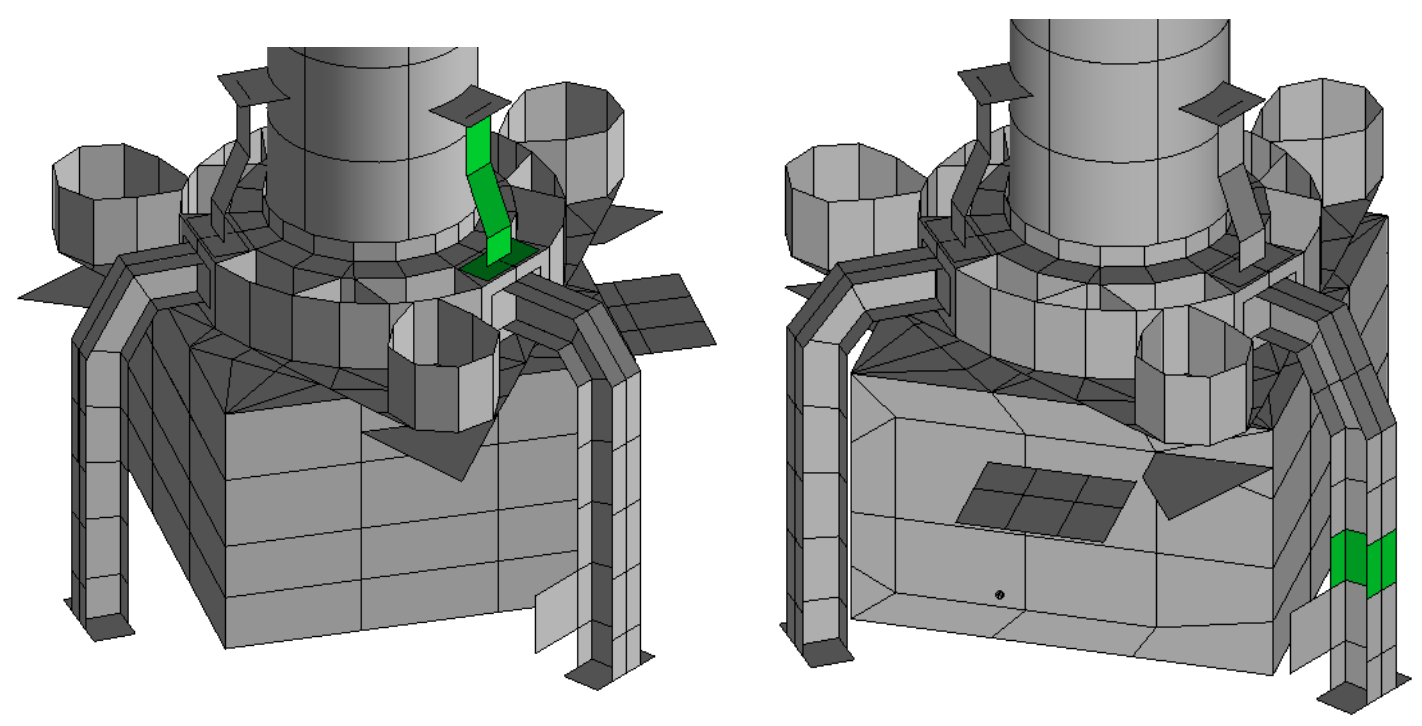

Fig. 5-26. RTMM condensed node no. 190 (left) and node no. 210 (right).

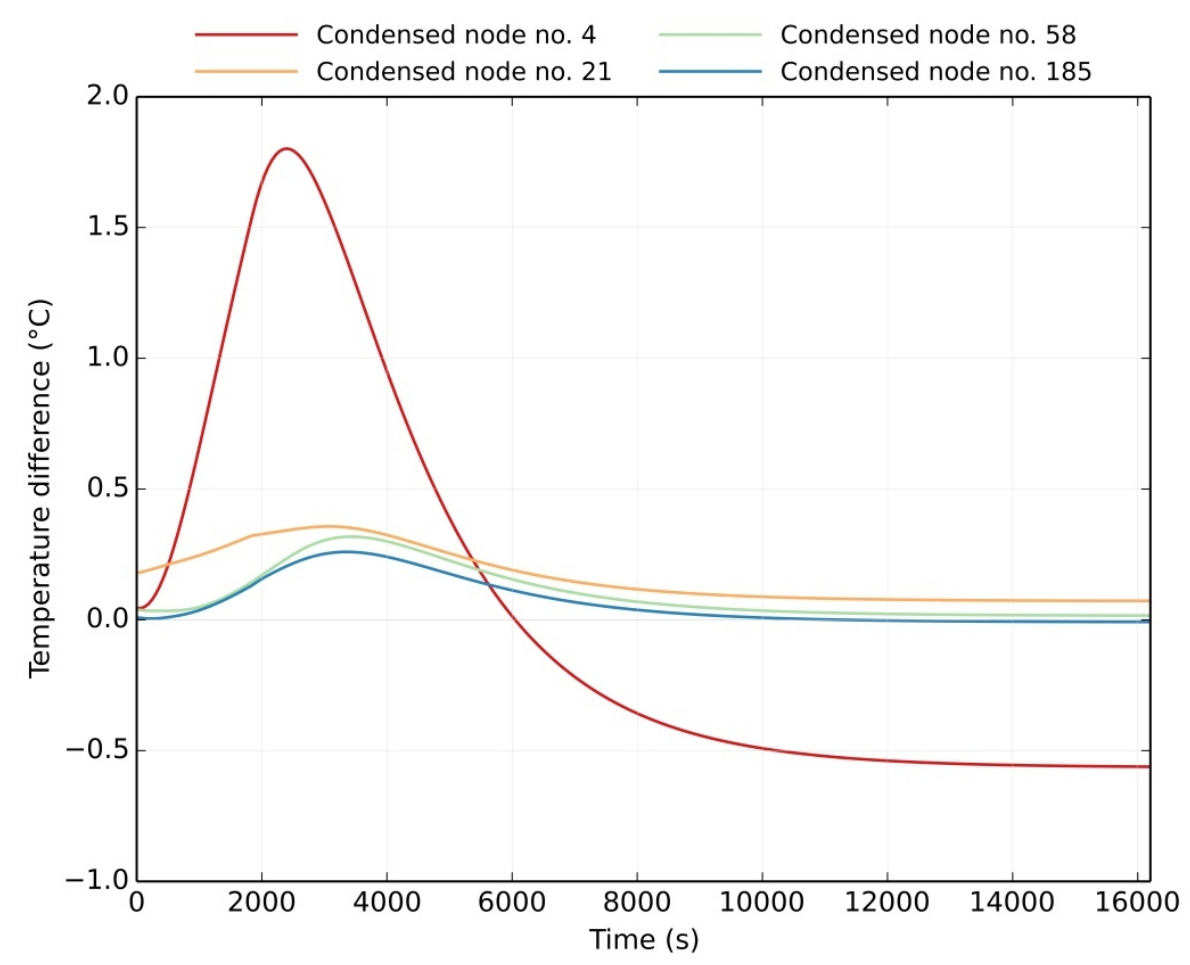

Fig. 5-27. Evolution of the temperatures of nodes 4, 21, 58 and 185. 

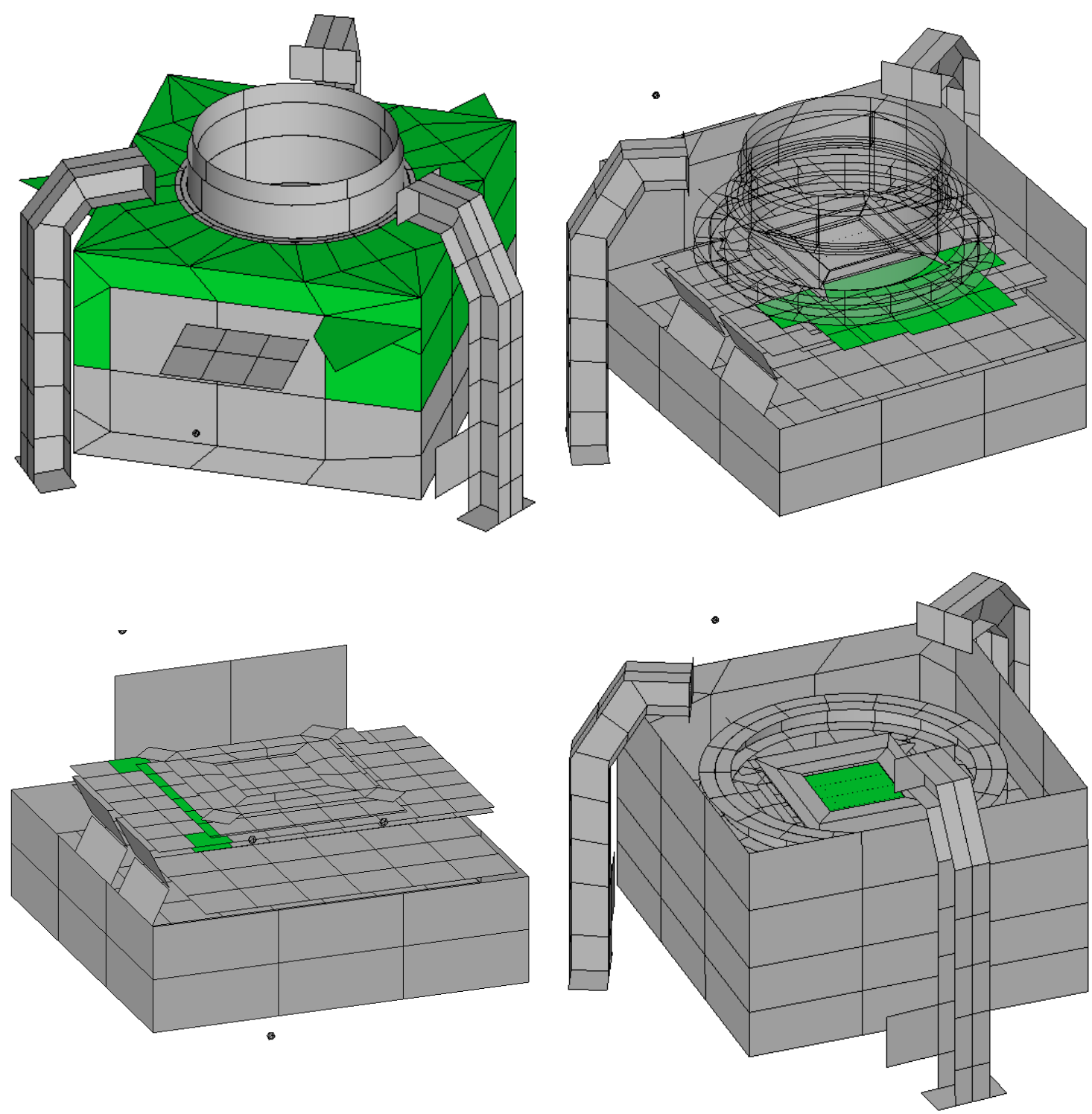

Fig. 5-28. RTMM condensed node no. 4 - housing upper lid (top left), no. 21 - cold finger (top right), no. 58 - sensor board (bottom left) and no. 185 - detector die (bottom right).

After $16200 \mathrm{~s}$, the system reached a quasi-steady state. For the RTMM, the maximum temperature variation over the last two hours was $0.03{ }^{\circ} \mathrm{C} / \mathrm{h}$ for the last hour and $0.30{ }^{\circ} \mathrm{C} / \mathrm{h}$ for the hour before. Regarding the DTMM, the maximum variations were $0.09{ }^{\circ} \mathrm{C} / \mathrm{h}$ and $0.67{ }^{\circ} \mathrm{C} / \mathrm{h}$ respectively. These values are compliant with the stability requirements given in $[77]^{12}$. In order to compare the results obtained with the reduced and the detailed model at the end of the

\footnotetext{
${ }^{12}$ This ECSS standard has been superseded by a newer standard (ECSS-E-ST-10-03C), which does not specify any numbers for the stabilization criteria. In theory, typical values should be included in a new handbook, but this has not been released at the time of writing this dissertation. As specified in Table 15 [77], the stability is reached when the temperatures have varied less than $1{ }^{\circ} \mathrm{C} / 1$ hour for at least a dwell time of 2 hours. The results are also compatible with different steady state conditions given by other authors, such as [88].
} 
transient analysis, one can repeat the same approach followed in section 5.2.1 to determine the temperature and heat fluxes differences. Fig. 5-29 shows the components for the temperature difference vector $\boldsymbol{\delta}$ at the end of the transient analysis (i.e. cold operational case). The comparison between the heat fluxes obtained in both models can be found in Table 5-6 and Table 5-7. The correlation results are worse than those shown in section 5.2.1 (for the hot operational steady state case, Fig. 5-20, Table 5-3 and Table 5-4), but, with the exception of the conductive heat flux from the FPA mounting interface (boundary node $i=2$ ), the values are within the required range.

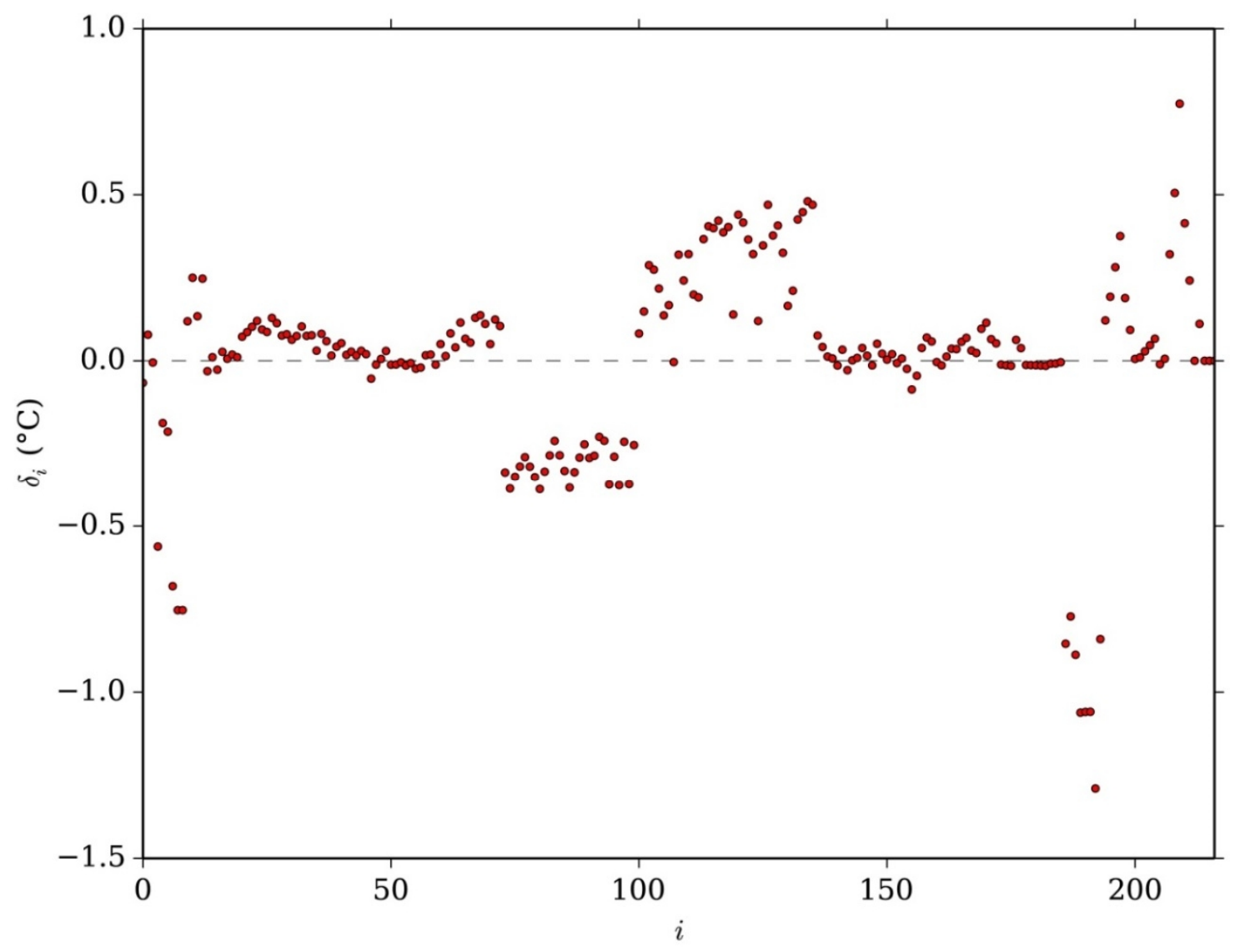

Fig. 5-29. Components of vector $\delta$ at the end of the transient analysis. 
Table 5-6. PHI FPA model conductive heat fluxes comparison at the end of the transient analysis. Orange cell indicates an unfulfillment of the required heat flux difference.

\begin{tabular}{c|c|c|c|c|}
$\begin{array}{c}i \\
\text { (boundary node) }\end{array}$ & 1 & 2 & 3 & 4 \\
\hline$H_{c b_{i}}^{D}(\mathrm{~W})$ & 1.81 & -0.85 & -0.14 & - \\
\hline$\hat{H}_{c b_{i}}^{R}(\mathrm{~W})$ & 1.81 & -1.01 & -0.14 & - \\
\hline$q_{c b_{i}} / \tilde{q}_{c b_{i}}$ & $<0.1 \%$ & $<18.9 \%$ & $<0.01 \mathrm{~W}$ & - \\
\hline
\end{tabular}

Table 5-7. PHI FPA model conductive heat fluxes comparison at the end of the transient analysis.

\begin{tabular}{c||c|c|c|c|}
$\begin{array}{c}i \\
\text { (boundary node) }\end{array}$ & 1 & 2 & 3 & 4 \\
\hline$H_{r b_{i}}^{D}(\mathrm{~W})$ & - & - & - & 1.62 \\
\hline$\hat{H}_{r b_{i}}^{R}(\mathrm{~W})$ & - & - & - & 1.77 \\
\hline$q_{r b_{i}} / \tilde{q}_{r b_{i}}$ & - & - & - & $<9.3 \%$
\end{tabular}




\section{CONCLUSIONS AND FUTURE WORK}

\subsection{Conclusions}

A method for spacecraft thermal model reduction has been developed. It is focused on internally mounted space scientific instrument LPM thermal mathematical models, although formally the method could be used in any other type of equipment. The method uses the steady state conditions (a model snapshot, in other words) for the reduction process, condensing the detailed model nodes based on thermal conductance values and temperature level similarities. While reducing the size of the detailed model, the technique presented here pursues the preservation of the physical characteristics and the topology of the original model (thermo-optical properties, thermal conductivities, global thermal capacity, symmetry of the matrices, conduction paths). Although the intention from the beginning was to build up a reduction technique fully automatic, a little intervention by the engineer is deemed necessary to choose at least values for the reduction ratio and maximum allowable heat flux and temperature differences.

The analysis made of the detailed model for node condensation gives some valuable information about the model structure. For instance, the numerical distribution of the conductors, how big are they in relation to those calculated with a reference value $\mu$, and where the meshing might be excessively detailed with regard to temperature gradients -there might be other reasons for using a detailed meshing, such as allowing ESATAN-TMS the automatic calculation of conductive couplings, see [78].

The value of the maximum reduction ratio $r_{r}$ for which the correlation is successful depends on the model characteristics and obviously the correlation criteria, but, according to the tests carried out, it seems quite difficult to achieve reductions with a reduction ratio of $90 \%$ or more. If it were necessary to reach higher levels of reduction, one possible option would be to tweak some reduced conductive couplings manually (following the classical approach for spacecraft thermal model reduction) or to use an automatic method for model correlation, in order to adjust some conductors and therefore improve the correlation. For automatic correlation methods, see for instance the software TAUMEL, based on an optimization algorithm and developed by Frey, Trinoga et al for Airbus Defense and Space [74, 75, 79]. Other techniques are presented by Torralbo et al [80], Jouffroy and Durand [81], Beck et al [82], Van Zijl et al [83], Anglada and Garmendia [76] and Klement [84, 85]. In case an automatic technique is used for improving the model correlation, it is important to keep the physical meaning of the model parameters. In our experience, the violation of the correlation requirements occurs typically for the heat fluxes through the boundaries. These violations might be corrected with minor changes in the reduced model conductors.

Regarding the reduction of the GMM, the need of assembling a RGMM will depend on the future use of the reduced thermal model. If the reduced thermal model is intended to be used in a higher hierarchy model for further analyses, or if it is planned to vary the thermo-optical properties on the RGMM, then of course it is necessary the RGMM building. However, if it were just planned to use for stand-alone calculations, then it would be enough to use the reduced radiative couplings matrix. 
The correlation errors (temperature differences, $\boldsymbol{\delta}$, and heat fluxes through the boundaries, $\mathbf{q}$ ) come from the range of the temperatures of the detailed nodes belonging to each reduced node. Radiative terms amplify the effect of this temperature differences among the condensed nodes. A second possible source of errors would be the different REFs obtained by setting up the RGMM, in comparison to those obtained by radiative couplings matrix reduction. The first source could be limited by refining the condensation algorithm, as it will be shown in the next sub-section Future work.

Regarding the filtering criteria for the processed conductive couplings matrix $\tilde{\mathbf{K}}^{\mathrm{I}}$, looking at the results shown in section 5.2.1, it seems that $p_{f}$ is less effective than $\Delta T_{\max }$. However, it is also possible that in the particular snapshot that is being used for the reduction, the temperatures of adjacent nodes are very similar, whereas in other cases, due to different heat sources, these very same nodes are farther from each other in terms of temperature levels. This effect could be then captured by filtering $\tilde{\mathbf{K}}^{\mathrm{I}}$ with dimensionless threshold $p_{f}$

Some of the limitations of the proposed methodology are:

- It is limited to LPM generated models.

- The software program is not able to deal with special thermal control technologies and features, such as thermostats and PID controlled heaters, Peltier coolers, and in general, any logic included in the thermal model. The program only parses the information contained in the ESATAN TMD file (see section 3). The logic can be manually implemented by the engineer, once the reduction is completed.

- One-way fluid conductors (GF conductors in ESATAN) are not parsed from the TMD ESATAN file, and therefore are not included in the reduction.

- The temperature dependency of some entities (conductivities, conductances, etc.) cannot be included as such in the reduction method.

- ESATAN sub-models are not supported by the program.

- The reduction of solids defined in ESATAN is tricky. The surface nodes in a solid geometry are arithmetic type, used solely for radiative analysis. Due to the exception handling, these nodes would be isolated in the reduction process.

- The way in which multi-layer insulation (MLI) is modelled could pose a limitation for model reduction. First, due to its relatively low thermal capacity, sometimes the MLI nodes can be modelled as arithmetic nodes, which would be isolated in the reduction process. Second, even in the case of having a very low thermal capacity (but not null), the shell thickness uses to be very small (typically around $0.1 \mathrm{~mm}$ ). So the MLI lateral conduction could be considered negligible, and not included in the computations. These two situations imply that in general, the MLI nodes belonging to the same side will not generally be condensed. 


\subsection{Future work}

The future work would be focused on improving the reduction process to achieve better results, and extending its application to transient analysis. Of course, other reduction approaches shall be investigated, but there is some space for enhancing the method presented in this text.

The temperature correlation errors calculated as presented in equation (48) check that all the components of $\boldsymbol{\delta}$ are smaller than $\delta_{\max }$. Another possible (and complementary) approach, as indicated in $[44,69]$, would be the standard deviation of the temperature differences. This could be easily implemented in the algorithm.

Some nodes could be directly isolated by the user, prior to starting the model reduction. These nodes would remain untouched in the reduction process. This could be interesting for some particular nodes, whose individual temperatures are of particular interest (for instance, a camera detector, electronics components, etc.). This would also ease the implementation of the logic present in the DTMM again in the RTMM (PID, thermostats).

The reduction method limits the temperature difference between two adjacent nodes, but it does not limit the temperature amplitude of the detailed model nodes as part of a reduced node. A possible way to limit the errors produced by this temperature range could be the clustering of the detailed nodes. This means, limiting the "size" of the reduced nodes according to the temperature range of the detailed nodes. Even more, the detailed model could be divided in different zones (for instance, depending on their relation with the radiative boundaries), and based on that, apply different temperature amplitude, $\Delta T_{\max }$ and $p_{f}$ thresholds for the reduction.

For the TMM reduction, the thermo-optical properties are not taken into account, but this information is provided by ESATAN in the TMD file (only infrared emissivity and solar absorptivity). The nodal association for the thermal model reduction could be limited to those nodes that have the same values of infrared emissivity and solar absorptivity (this does not guarantee that they have the same thermo-optical property, but it is very likely to be the case). The bulk properties data (thermal conductivity, specific heat capacity and density) is not copied to the TMD file by ESATAN. However, it could be possible to parse other model files in order to get this information. With this data available, the reduction process could be improved, by refining the calculation of $\mathbf{K}^{\mathrm{S}}$. As it is defined now, $\mu$ only provides qualitative information of the conductors of the thermal model. However, if different values of $\mu$ were used for the determination of $\mathbf{K}^{\mathrm{S}}$, it would allow, for instance, to compare more effectively the conductors in a zone. Thus, being able to identify which conductors corresponds to contacts and which to solid material.

The reduction of the MLI can be very tricky, as mentioned before. One option to address this limitation would be to identify patterns in the couplings matrices, since the structure of the MLI related couplings is very particular.

Finally, although the reduction method is mainly based on physical properties of the model, the temperature differences among the nodes are driven also by the heat sources and the boundary temperatures. Section 5.2.3 showed that the reduced model using hot conditions behaves fairly well in cold conditions. However, if the cold conditions snapshot were also used in the reduction, the obtained reduced model would be more consistent with broader set of scenarios. Of course the RTMM resulted from both snapshots should be the same. Thus, both adjacency 
matrices should be overlapped, preceding the zeros over the ones. In principle, any number of snapshots could be used.

For the reduction algorithm presented in this dissertation, it has been used a single temperature difference correlation criterion. Different zones of the detailed model could be reduced following different correlation error criteria (external nodes, MLI nodes, etc.) as suggested for instance in [69]. These zones could be identified by the program, again finding some patterns in the matrices (not only "detecting" MLI nodes; also with the REFs, the areas and the radiative environment node would be possible to identify the outer "shell" of the model). Node ranges and labels could be used to this end as well (clustering the model), but in order to make the method as universal as possible, this should be implemented carefully, as it would require some previous work by the engineer.

As previously discussed, many aspects can be improved in the reduction process. This work takes the first step, defining a methodology that has been tested in two models, showing a good correlation level while saving a significant amount of computation time. 


\section{REFERENCES}

[1] D.G. Gilmore, ed., Spacecraft Thermal Control Handbook. Volume 1: Fundamental Technologies, 2nd ed., The Aerospace Press, El Segundo, California, 2002. doi:10.2514/4.989117.

[2] J. Meseguer, I. Pérez-Grande, A. Sanz-Andrés, Spacecraft Thermal Control, Elsevier, 2012. doi:10.1533/9780857096081.

[3] J. Meseguer, I. Pérez-Grande, A. Sanz-Andrés, G. Alonso, Thermal Systems, in: M. Macdonald, V. Badescu (Eds.), Int. Handb. Sp. Technol., 2014: pp. 371-396. doi:10.1007/978-3-642-41101-4.

[4] C.J. Savage, Thermal Control of Spacecraft, in: Spacecr. Syst. Eng., 2011: pp. 357-394. doi:10.1002/9781119971009.ch11.

[5] ECSS Standard: Space engineering - System engineering general requirements (ECSS-EST-10C Rev.1), ESA Requirements and Standards Division, 2017.

[6] A.R.L. Tatnall, J.B. Farrow, M. Bandecchi, C.R. Francis, Spacecraft System Engineering, in: P. Fortescue, G.G. Swinerd, J. Stark (Eds.), Spacecr. Syst. Eng. Fourth Ed., John Wiley \& Sons, Ltd, 2011: pp. 643-678. doi:10.1002/9781119971009.ch20.

[7] K. Oshima, Y. Oshima, An analytical approach to the thermal design of spacecrafts, ISAS Rep. 33(1). http://airex.tksc.jaxa.jp/dr/prc/japan/contents/IS2400575000/IS2400575.pdf （accessed March 6, 2013).

[8] I. Pérez-Grande, A. Sanz-Andrés, C. Guerra, G. Alonso, Analytical study of the thermal behaviour and stability of a small satellite, Appl. Therm. Eng. 29 (2009) 2567-2573. doi:10.1016/j.applthermaleng.2008.12.038.

[9] C. Arduini, G. Laneve, S. Folco, Linearized techniques for solving the inverse problem in the satellite thermal control, Acta Astronaut. 43 (1998) 473-479. doi:10.1016/S00945765(98)00180-5.

[10] J. Gaite, Nonlinear analysis of spacecraft thermal models, Nonlinear Dyn. 65 (2011) 283-300. doi:10.1007/s11071-010-9890-4.

[11] J. Gaite, A. Sanz-Andrés, I. Pérez-Grande, Nonlinear analysis of a simple model of temperature evolution in a satellite, Nonlinear Dyn. 58 (2009) 405-415. doi:10.1007/s11071-009-9488-x.

[12] M. a. Gadalla, Prediction of temperature variation in a rotating spacecraft in space environment, Appl. Therm. Eng. $25 \quad$ (2005) 2379-2397. doi:10.1016/j.applthermaleng.2004.12.018.

[13] J.-R. Tsai, Overview of Satellite Thermal Analytical Model, J. Spacecr. Rockets. 41 (2004) 120-125. doi:10.2514/1.9273.

[14] S. Bishop, F. Castro, C. Goulding, D. Presti, A. Woodcock, Implementation and validation of a PC based ESATAN model for the Ulysses spacecraft, in: Therm. Fluids Anal. Work., NASA, Orlando, FL, 2005.

[15] D. Müller, R.G. Marsden, O.C. St. Cyr, H.R. Gilbert, Solar Orbiter: Exploring the SunHeliosphere Connection, 2013. doi:10.1007/s11207-012-0085-7.

[16] ITP Engines UK Ltd., ESATAN-TMS Thermal User Manual, (2014). 
[17] A. Gandorfer, S.K. Solanki, J. Woch, V.M. Pillet, A.Á. Herrero, T. Appourchaux, The Solar Orbiter Mission and its Polarimetric and Helioseismic Imager (SO/PHI), J. Phys. Conf. Ser. 271 (2011). doi:10.1088/1742-6596/271/1/012086.

[18] B. Besselink, U. Tabak, A. Lutowska, N. Van De Wouw, H. Nijmeijer, D.J. Rixen, M.E. Hochstenbach, W.H.A. Schilders, A comparison of model reduction techniques from structural dynamics, numerical mathematics and systems and control, J. Sound Vib. 332 (2013) 4403-4422. doi:10.1016/j.jsv.2013.03.025.

[19] W.H.A. Schilders, H.A. Van Der Vorst, J. Rommes, Model Order Reduction: Theory, Research Aspects and Applications, Springer Berlin Heidelberg, Berlin, Heidelberg, 2008. doi:10.1007/978-3-540-78841-6.

[20] S. Djukic, A. Saric, Dynamic model reduction: An overview of available techniques with application to power systems, Serbian J. Electr. Eng. 9 (2012) 131-169. doi:10.2298/SJEE1202131D.

[21] D. De Klerk, D.J. Rixen, S.N. Voormeeren, General Framework for Dynamic Substructuring: History, Review and Classification of Techniques, AIAA J. 46 (2008) 1169-1181. doi:10.2514/1.33274.

[22] R.J. Guyan, Reduction of stiffness and mass matrices, AIAA J. 3 (1965) 380-380. doi:10.2514/3.2874.

[23] M. Bernard, A thermal model reduction tool, in: 23th Eur. Work. Therm. ECLS Softw., ESA/ESTEC, Noordwijk, The Netherlands, 2009: pp. 53-68. https://exchange.esa.int/thermalworkshop/attachments/workshop2009/appendix/TMRT.pdf.

[24] M. Bernard, J. Etchells, T. Basset, F. Brunetti, Thermal Model Reduction - Theory \&amp; Application, in: 40th Int. Conf. Environ. Syst., American Institute of Aeronautics and Astronautics, Reston, Virigina, 2010. doi:10.2514/6.2010-6084.

[25] M. Bernard, T. Basset, J. Etchells, Thermal Model Reduction Tool, in: 24th Eur. Work. Therm. ECLS Softw., ESA/ESTEC, Noordwijk, The Netherlands, 2010: pp. 169-180. https:/exchange.esa.int/thermal-workshop/attachments/workshop2010/parts/TMRT.pdf.

[26] D. Kintea, Model reduction of Sentinel 1, in: 25th Eur. Work. Therm. ECLS Softw., ESA/ESTEC, Noordwijk, The Netherlands, 2011: pp. 295-308.

[27] T. Basset, P. Hugonnot, P. Connil, M. Ferrier, TMRT (Thermal Model Reduction Tool): Presentation of the tool and application on satellite model reduction for launcher coupled analysis, in: 46th Int. Conf. Environ. Syst., American Institute of Aeronautics and Astronautics, Vienna, Austria, 2016. http://hdl.handle.net/2346/67549.

[28] P. Heres, Robust and efficient Krylov subspace methods for Model Order Reduction, Eindhoven University of Technology, 2005.

[29] M. Rewienski, J. White, A trajectory piecewise-linear approach to model order reduction and fast simulation of nonlinear circuits and micromachined devices, IEEE Trans. Comput. Des. Integr. Circuits Syst. 22 (2003) 155-170. doi:10.1109/TCAD.2002.806601.

[30] Y. Wang, H. Song, K. Pant, H. Peabody, J. Ku, C.D. Butler, A projection-based model order reduction simulation tool for spacecraft thermal analysis, in: Therm. Fluids Anal. Work., NASA, Newport News, 2011. http://tfaws.nasa.gov/TFAWS11/Proceedings/TFAWS2011-PT-001.pdf.

[31] J. Qian, Y. Wang, H. Song, K. Pant, H. Peabody, J. Ku, C.D. Butler, Projection-Based Reduced-Order Modeling for Spacecraft Thermal Analysis, J. Spacecr. Rockets. 52 (2015) 978-989. doi:10.2514/1.A33117. 
[32] M. Deiml, M. Suderland, P. Reiss, M. Czupalla, Development and evaluation of thermal model reduction algorithms for spacecraft, Acta Astronaut. 110 (2015) 168-179. doi:10.1016/j.actaastro.2015.01.018.

[33] Y.J. Yang, K.Y. Shen, Nonlinear heat-transfer macromodeling for MEMS thermal devices, J. Micromechanics Microengineering. 15 (2005) 408-418. doi:10.1088/0960$1317 / 15 / 2 / 022$.

[34] M.J. Rewienski, A Trajectory Piecewise-Linear Approach to Model Order Reduction of Nonlinear Dynamical Systems, Technical University of Gdansk, 2003.

[35] A. Chatterjee, An Introduction to the Proper Orthogonal Decomposition, IEEE Photonics Technol. Lett. 78 (2000) 808-817. http://www.jstor.org/stable/24103957.

[36] L. Sirovich, Turbulence and the dynamics of coherent structures. I-III, Q. Appl. Math. 45 (1987) 561-590. http://www.jstor.org/stable/43637457.

[37] R. Pinnau, Model Reduction via Proper Orthogonal Decomposition, in: Model Order Reduct. Theory, Res. Asp. Appl., 2008: pp. 95-109. doi:10.1007/978-3-540-78841-6_5.

[38] P. Benner, S. Gugercin, K. Willcox, A Survey of Projection-Based Model Reduction Methods for Parametric Dynamical Systems, SIAM Rev. 57 (2015) 483-531. doi:10.1137/130932715.

[39] A. Antoulas, D. Sorensen, S. Gugercin, A survey of model reduction methods for largescale systems, Contemp. Math. $280 \quad$ (2001) 193-220. doi:http://dx.doi.org/10.1090/conm/280.

[40] D. Celo, X. Guo, P.K. Gunupudi, R. Khazaka, D.J. Walkey, T. Smy, M.S. Nakhla, The creation of compact thermal models of electronic components using model reduction, IEEE Trans. Adv. Packag. 28 (2005) 240-251. doi:10.1109/TADVP.2005.846942.

[41] F. Jouffroy, D. Charvet, M. Jacquiau, A. Capitaine, Automated thermal model reduction for telecom S/C walls, in: 18th Eur. Work. Therm. ECLS Softw., ESA/ESTEC, Noordwijk, The Netherlands, 2004.

[42] L.B. Bushard, On the value of Guyan Reduction in dynamic thermal problems, Comput. Struct. 13 (1981) 525-531. doi:10.1016/0045-7949(81)90047-X.

[43] M. Molina, C. Clemente, Thermal Model Automatic Reduction: algorithm and validation techniques, in: 19th Eur. Work. Therm. ECLS Softw., ESA/ESTEC, Noordwijk, The Netherlands, 2005.

[44] M. Molina, C. Clemente, Thermal Model Automatic Reduction: Algorithm and Validation Techniques, in: 36th Int. Conf. Environ. Syst., SAE International, Savannah, Georgia, 2006. doi:10.4271/2006-01-2112.

[45] J.H. Kim, B. Kim, Study on the reduction method of the satellite thermal mathematical model, Adv. Eng. Softw. 108 (2017) 37-47. doi:10.1016/j.advengsoft.2017.02.007.

[46] G. Fernández-Rico, I. Pérez-Grande, A. Sanz-Andres, I. Torralbo, J. Woch, Quasiautonomous thermal model reduction for steady-state problems in space systems, Appl. Therm. Eng. 105 (2016) 456-466. doi:10.1016/j.applthermaleng.2016.03.017.

[47] S. Krishnamoorthy, M.H. Chowdhury, Compact thermal network model: Realization and reduction, in: IEEE Int. Conf. Electro/Information Technol., 2008: pp. 389-394. doi:10.1109/EIT.2008.4554334.

[48] A.K. Noor, C.D. Balch, M.A. Shibut, Reduction methods for nonlinear steady-state thermal analysis, Int. J. Numer. Methods Eng. 20 (1984) 1323-1348. doi:10.1002/nme.1620200711. 
[49] M. Gorlani, Thermal Model Reduction with Stochastic Optimisation, in: 20th Eur. Work. Therm. ECLS Softw., ESA/ESTEC, Noordwijk, The Netherlands, 2006: pp. 189-198.

[50] M. Gorlani, M. Rossi, Thermal Model Reduction with Stochastic Optimisation, in: 37th Int. Conf. Environ. Syst., SAE International, Chicago, Illinois, 2007. doi:10.4271/200701-3119.

[51] S. Schmidt, L. Kreusser, S. Zhang, POD-DEIM based model order reduction for a threedimensional microscopic Li-Ion battery model, Kaiserslautern, 2013.

[52] P.T. Boggs, J.W. Tolle, Sequential Quadratic Programming, Acta Numer. 4 (1995) 1-51. doi:10.1017/S0962492900002518.

[53] A.I.F. Vaz, L.N. Vicente, PSwarm: A hybrid solver for linearly constrained global derivative-free optimization, Optim. Methods Softw. 24 (2009) 669-685. doi:10.1080/10556780902909948.

[54] J.M.A. Scherpen, Balancing for nonlinear systems, Syst. Control Lett. 21 (1993) 143153. doi:https://doi.org/10.1016/0167-6911(93)90117-O.

[55] D.W. Hengeveld, Reduced-Order Modeling for Rapid Thermal Analysis and Evaluation of Spacecraft, in: 46th AIAA Thermophys. Conf., American Institute of Aeronautics and Astronautics, Reston, Virginia, 2016. doi:10.2514/6.2016-3698.

[56] D.W. Hengeveld, A. Biskner, Enhanced data exploration through Reduced-Order Models, in: 47th Int. Conf. Environ. Syst., American Institute of Aeronautics and Astronautics, Charleston, South Carolina, 2017. http:/hdl.handle.net/2346/72901.

[57] L. Jacques, E. Béchet, G. Kerschen, Finite element model reduction for space thermal analysis, Finite Elem. Anal. Des. 127 (2017) 6-15. doi:10.1016/j.finel.2017.01.001.

[58] J.H. Chin, T.D. Panczak, L. Fried, Spacecraft thermal modelling, Int. J. Numer. Methods Eng. 35 (1992) 641-653. doi:10.1002/nme.1620350403.

[59] ITP Engines UK Ltd., ESATAN-TMS Thermal Engineering Manual, (2014).

[60] N. van der Meijs, Model Order Reduction of Large RC Circuits, in: W.A. Schilders, H. van der Vorst, J. Rommes (Eds.), Model Order Reduct. Theory, Res. Asp. Appl., Springer Berlin Heidelberg, 2008: pp. 421-446. doi:10.1007/978-3-540-78841-6_19.

[61] J. Rommes, W.H.A. Schilders, Efficient methods for large resistor networks, IEEE Trans. Comput. Des. Integr. Circuits Syst. 29 (2010) 28-39. doi:10.1109/TCAD.2009.2034402.

[62] R. Tarjan, Depth-first search and linear graph algorithms, 12th Annu. Symp. Switch. Autom. Theory (Swat 1971). 1 (1971) 146-160. doi:10.1109/SWAT.1971.10.

[63] T. Lengauer, R.E. Tarjan, A fast algorithm for finding dominators in a flowgraph, ACM Trans. Program. Lang. Syst. 1 (1979) 121-141. doi:10.1145/357062.357071.

[64] D.J. Pearce, An Improved Algorithm for Finding the Strongly Connected Components of a Directed Graph, 2005. http://homepages.ecs.vuw.ac.nz/ djp/files/P05.pdf.

[65] J.P. Holman, Heat Transfer, 10th ed., McGraw Hill, New York, 2012.

[66] S. Appel, R. Patrício, H.P. de Koning, O. Pin, Automatic Conductor Generation for Thermal Lumped Parameter Models, in: 34th Int. Conf. Environ. Syst., SAE International, Colorado Springs, Colorado, 2004. doi:10.4271/2004-01-2397.

[67] C.J. Kirtley, N.J. Stock, H.P. De Koning, S. Appel, E.S.A. Estec, Automatic Linear Conductor Generation Solution for Lumped Parameter Models, in: 35th Int. Conf. Environ. Syst., SAE International, Rome, Italy, 2005. doi:10.4271/2005-01-3059. 
[68] A. Buluç, J. Gilbert, V.B. Shah, Implementing Sparse Matrices for Graph Algorithms, in: Graph Algorithms Lang. Linear Algebr., 2011: pp. 287-313. doi:10.1137/1.9780898719918.ch13.

[69] ECSS Handbook: Space engineering - Thermal analysis handbook (ECSS-E-HB-3103A), ESA Requirements and Standards Division, 2016.

[70] W. Ley, K. Wittmann, W. Hallmann, eds., Handbook of Space Technology, John Wiley \& Sons, Ltd, Chichester, UK, 2009. doi:10.1002/9780470742433.

[71] J. Gaite, G. Fernandez-Rico, Linear Approach to the Orbiting Spacecraft Thermal Problem, J. Thermophys. Heat Transf. 26 (2012) 511-522. doi:10.2514/1.T3748.

[72] I. Pérez Grande, I. Torralbo, G. Alonso, A. Gómez-Sanjuan, G. Fernandez-Rico, Thermal Design of the ESA Solar Orbiter Instrument PHI, in: 46th Int. Conf. Environ. Syst., American Institute of Aeronautics and Astronautics, Vienna, Austria, 2016. https://ttu-ir.tdl.org/ttu-ir/handle/2346/67685.

[73] J.D. Hunter, Matplotlib: A 2D graphics environment, Comput. Sci. Eng. 9 (2007) 99104. doi:10.1109/MCSE.2007.55.

[74] B. Frey, N. Bohne, M. Bruno, Development, Testing and Benchmarking of an Automated Thermal Model Correlation Tool, in: 46th Int. Conf. Environ. Syst., American Institute of Aeronautics and Astronautics, Vienna, Austria, 2016: p. 14.

[75] M. Trinoga, Automated thermal model correlation, in: 30th Eur. Sp. Therm. Anal. Work., ESA/ESTEC, Noordwijk, The Netherlands, 2016: pp. 77-92.

[76] E. Anglada, I. Garmendia, Correlation of thermal mathematical models for thermal control of space vehicles by means of genetic algorithms, Acta Astronaut. 108 (2015) 117. doi:10.1016/j.actaastro.2014.11.042.

[77] ECSS Standard: Space engineering - Testing (ECSS-E-10-03A), ESA Requirements and Standards Division, 2002.

[78] ITP Engines UK Ltd., ESATAN-TMS Workbench User Manual, (2014).

[79] B. Frey, M. Trinoga, M. Hoppe, W.-D. Ebeling, Development of an Automated Thermal Model Correlation Method and Tool, in: 45h Int. Conf. Environ. Syst., American Institute of Aeronautics and Astronautics, Bellevue, Washington, 2015.

[80] I. Torralbo, I. Perez-Grande, A. Sanz-Andres, J. Piqueras, Correlation of spacecraft thermal mathematical models to reference data, Acta Astronaut. (2017). doi:10.1016/j.actaastro.2017.12.033.

[81] F. Jouffroy, N. Durand, Thermal model correlation using Genetic Algorithms, in: 21th Eur. Work. Therm. ECLS Softw., ESA/ESTEC, Noordwijk, The Netherlands, 2007: pp. 127-137.

[82] T. Beck, A. Bieler, N. Thomas, Numerical thermal mathematical model correlation to thermal balance test using adaptive particle swarm optimization (APSO), Appl. Therm. Eng. 38 (2012) 168-174. doi:10.1016/j.applthermaleng.2012.01.027.

[83] N. Van Zijl, B. Zandbergen, B. Benthem, Correlating thermal balance test results with a thermal mathematical model using evolutionary algorithms, in: 27th Eur. Sp. Therm. Anal. Work., ESA/ESTEC, Noordwijk, The Netherlands, 2013: pp. 89-108.

[84] J. Klement, On using quasi Newton algorithms of the Broyden class for model-to-test correlation, in: 28th Eur. Sp. Therm. Anal. Work., ESA/ESTEC, Noordwijk, The Netherlands, 2014: pp. 213-228.

[85] J. Klement, On Using Quasi-Newton Algorithms of the Broyden Class for Model-to-Test Correlation, J. Aerosp. Technol. Manag. 6 (2014) 407-414. doi:10.5028/jatm.v6i4.373. 
[86] U. Rauscher, Guidelines for high accuracy thermal modelling. Experiences and results from ESA study: improvement of thermal analysis accuracy, in: 21th Eur. Work. Therm. ECLS Softw., ESA/ESTEC, Noordwijk, The Netherlands, 2007.

[87] ECSS Standard: Space engineering - Thermal control general requirements (ECSS-EST-31C), ESA Requirements and Standards Division, 2008.

[88] N. Lynch, D. Boyd, M. Freeman, Precollimators: passive on-orbit thermal control for space based telescope apertures, in: Sixth Eur. Symp. Sp. Environ. Control Syst., ESA Publications Division, Noordwijk, The Netherlands, 1997: pp. 175-182. 


\section{AnNeX}

\subsection{Example of poor exception handling}

As indicated in 4.2.1, the nodes that have a zero thermal capacity (arithmetic nodes) are treated as isolated nodes. This might pose a problem, if a thermal model has a big number of them, since the amount of these nodes will limit the reduction ratio that can be achieved. For instance, the Solar Orbiter PHI Electronics Unit thermal model has 1571 arithmetic nodes (they correspond to the electronic component cases). Fig. 8-1 shows the maximum reduction ratio $\left(r_{r}=0.75\right)$ reachable using the method as it is, which is a low value if compared to maximum theoretical calculated for PHI FPA, see Fig. 5-9. Therefore, the exception handling should be treated in a different way for the cases of high ratio of arithmetic nodes.

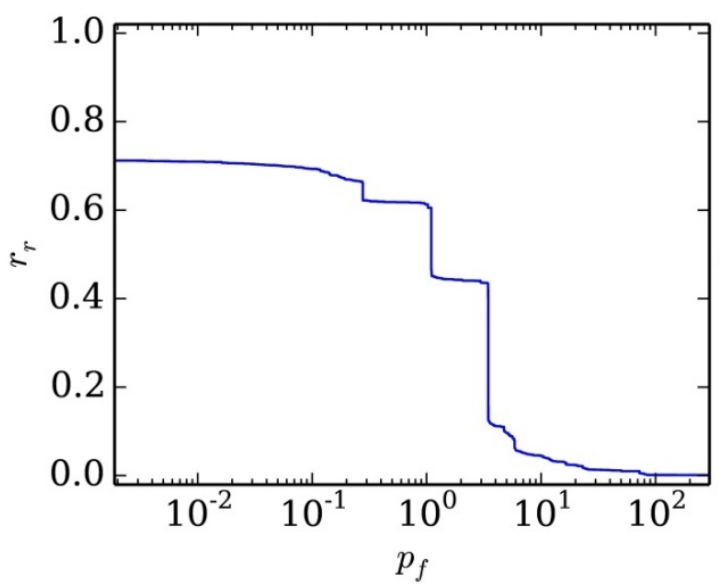

(a)

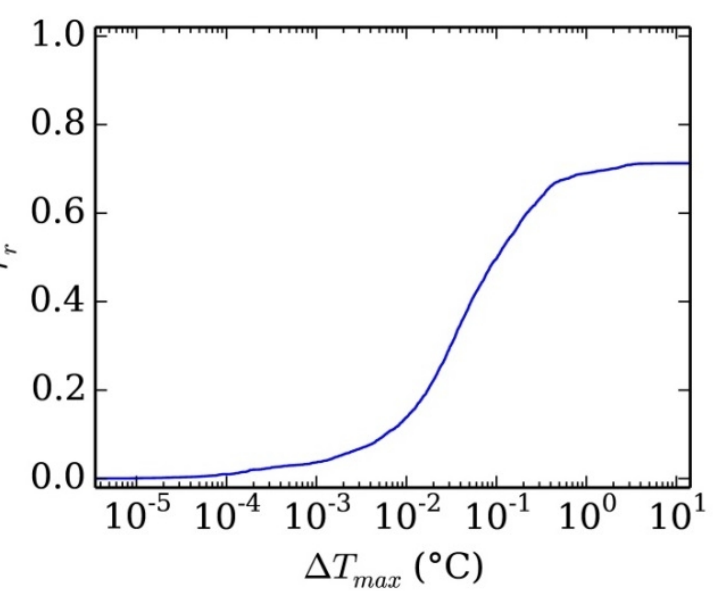

(b)

Fig. 8-1. SO/PHI Electronics Unit: variation of (a) $r_{r}$ vs. $p_{f}$, and variation of (b) $r_{r}$ vs. $\Delta T_{\max }$. For (a), the SCC algorithm is run with $\tilde{K}^{1, F}$. For (b), the matrix $K$ is multiplied element-wise by $\Theta^{F}$, and the SCC algorithm is run with the resulting matrix. 


\subsection{Output of the PHI OPT FPA model reduction (extract)}

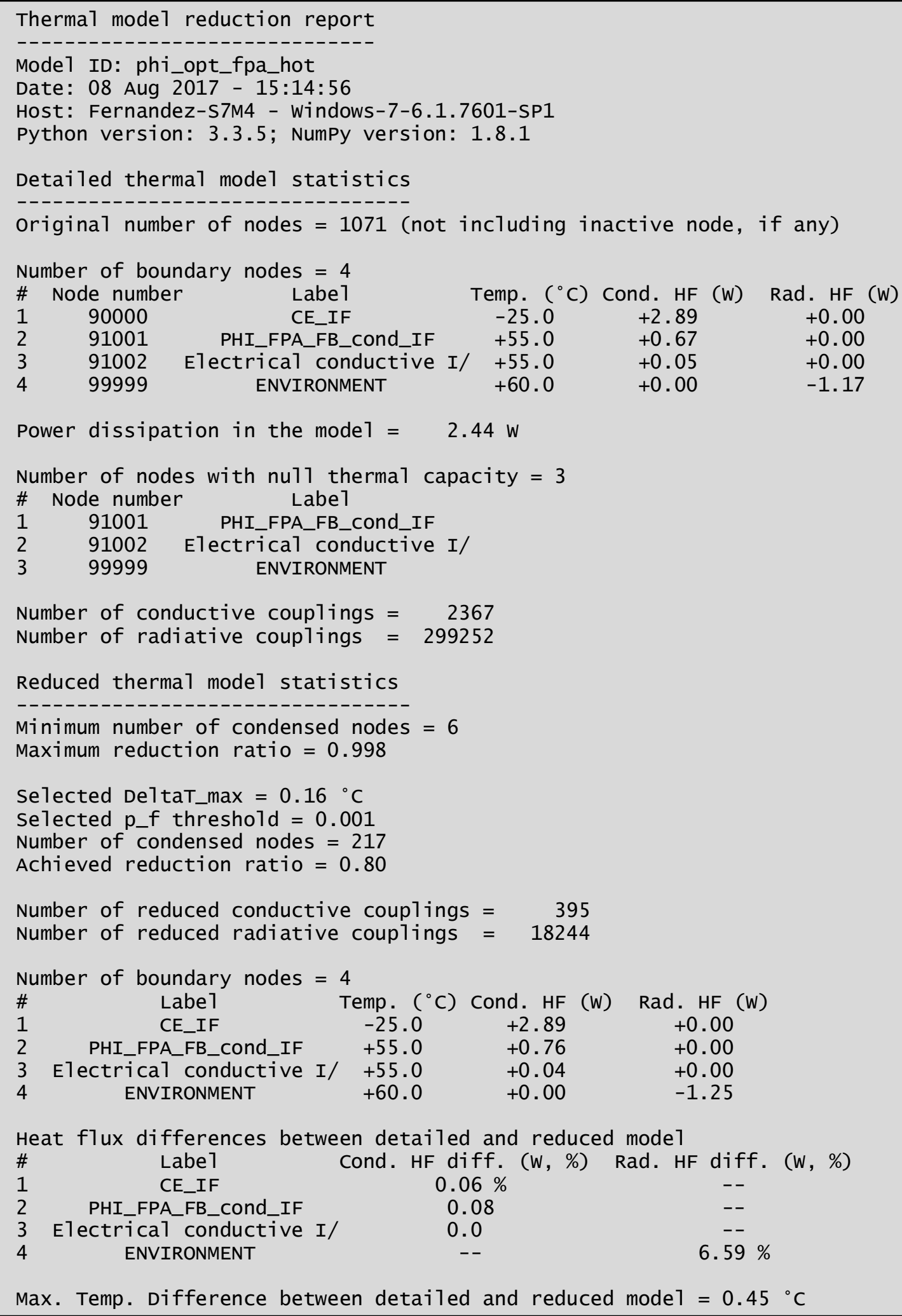




\subsection{TMD files structure}

For the Solar Orbiter PHI FPA test case (see section 5.2), a TMD file is used as input (TMD information is taken as reference, as shown in section 3). The program in which the reduction method has been implemented produces in turn a TMD file that can be opened in ESATAN, in order to visualize the reduced nodes and the temperature differences. Both TMD files structures are shown below. The structure of a HDF file (such as the TMD abovementioned) can be seen with a HDF viewer ${ }^{13}$.

\subsubsection{ESATAN-TMS produced TMD file (as of release 7 service pack 2)}

\begin{tabular}{|c|c|c|}
\hline Name & Kind & Data type and description \\
\hline AnalysisSet1 & HDF5 Group & \\
\hline DataGroup1 & HDF5 Group & \\
\hline conductorDataGL & HDF Dataset & 8-byte floating point, conductive couplings matrix \\
\hline conductorDataGR & HDF Dataset & 8-byte floating point, radiative couplings matrix \\
\hline thermalNodesRealData & HDF Dataset & $\begin{array}{l}\text { 8-byte floating point, numerical values for } \\
\text { categories defined by thermalNodesRealAttributes }\end{array}$ \\
\hline the thermalNodesStringData & HDF Dataset[text] & $\begin{array}{l}\text { String values for categories defined by } \\
\text { thermalStringRealAttributes }\end{array}$ \\
\hline Himes & HDF Dataset & $\begin{array}{l}\text { 8-byte floating point, time vector (single zero value } \\
\text { for steady state case) }\end{array}$ \\
\hline conductorsGL & HDF Dataset & $\begin{array}{l}\text { 4-byte signed integer, defines the node pairs for the } \\
\text { Dataset conductorDataGL }\end{array}$ \\
\hline conductorsGR & HDF Dataset & $\begin{array}{l}\text { 4-byte signed integer, defines the node pairs for the } \\
\text { Dataset conductorDataGR }\end{array}$ \\
\hline models & HDF Dataset & $\begin{array}{l}\text { Compound, indicates model id. and sub-model } \\
\text { structure }\end{array}$ \\
\hline thermalNodes & HDF Dataset & $\begin{array}{l}\text { 4-byte signed integer, defines node numbers and } \\
\text { their assignation to the different sub-models }\end{array}$ \\
\hline abs thermalNodesRealAttributes & HDF Dataset[text] & $\begin{array}{l}\text { Define the categories }{ }^{14} \text { for the } \\
\text { thermalNodesRealData Dataset }\end{array}$ \\
\hline abo thermalNodesStringAttributes & HDF Dataset[text] & $\begin{array}{l}\text { Define the categories for the } \\
\text { thermalNodesStringData Dataset (Type and Label) }\end{array}$ \\
\hline
\end{tabular}

${ }^{13}$ The HDF Group provides an open-source multiplatform HDF files viewer in their web page: https://support.hdfgroup.org/projects/compass/download.html

${ }^{14}$ These are Temperature, Capacitance, Total Albedo Heat Source, Total Earth Heat Source, Total Internal Heat Source, Total Rest Heat Source, Total Solar Heat Source, Area, Solar Absorptivity, InfraRed Emissivity, Incident Albedo Heat Source, Incident Earth Heat Source, Incident Solar Heat Source, X Coordinate, Y Coordinate, Z Coordinate. 
8.3.2 TMD file output by the reduction software

\begin{tabular}{|c|c|c|}
\hline Name & Kind & Data type and description \\
\hline AnalysisSet1 & HDF5 Group & \\
\hline DataGroup1 & HDF5 Group & \\
\hline thermalNodesRealData & HDF Dataset & $\begin{array}{l}\text { 8-byte floating point, numerical values for } \\
\text { categories defined by thermalNodesRealAttributes }\end{array}$ \\
\hline ab thermalNodesStringData & HDF Dataset[text] & $\begin{array}{l}\text { String values for categories defined by } \\
\text { thermalStringRealAttributes }\end{array}$ \\
\hline times & HDF Dataset & $\begin{array}{l}\text { 8-byte floating point, time vector (single zero value } \\
\text { for steady state case) }\end{array}$ \\
\hline models & HDF Dataset & $\begin{array}{l}\text { Compound, indicates model id. and sub-model } \\
\text { structure }\end{array}$ \\
\hline thermalNodes & HDF Dataset & $\begin{array}{l}\text { 4-byte signed integer, defines node numbers and } \\
\text { their assignation to the different sub-models }\end{array}$ \\
\hline abo thermalNodesRealAttributes & HDF Dataset[text] & $\begin{array}{l}\text { Define the categories }{ }^{15} \text { for the } \\
\text { thermalNodesRealData Dataset }\end{array}$ \\
\hline abo thermalNodesStringAttributes & HDF Dataset[text] & $\begin{array}{l}\text { Define the categories for the } \\
\text { thermalNodesStringData Dataset (Type and Label) }\end{array}$ \\
\hline
\end{tabular}

15 These are DTMM Temperature, Reduced node number, RTMM Temperature, DTMM-RTMM Temperature difference $(\boldsymbol{\tau})$. 




\title{
CURRICULUM VitAE
}

\author{
Germán Fernández Rico \\ Date of birth: 09 March 1983 \\ Nationality: Spanish \\ E-mail: ger.fdz.rico at gmail.com
}

\section{PROFESSIONAL EXPERIENCE}

Jul 2014 Thermal engineer at Max Planck Institute for Solar System Research

- present (Göttingen, Germany)

- Thermal systems engineering for Sunrise 3, a balloon-borne solar observatory.

- Thermal analysis, design and testing of subsystems for PHI and METIS instruments on board Solar Orbiter ESA mission.

- Test lead of PHI instrument flight model thermal test campaign.

- Flight thermal hardware procurement and integration.

- Support to general AIT activities in clean room environment.

Sep 2011 Assistant Teacher at Universidad Politécnica de Madrid, UPM

- Jul 2014 - Course: aerospace technology.

- Collaborating with Instituto Universitario de Microgravedad "Ignacio Da

Riva" (IDR/UPM) in thermal modelling and meeting attendance for ESA projects, mainly Solar Orbiter PHI.

- Thermal testing in the thermal vacuum chamber at IDR/UPM.

- Integration of the educational Concurrent Design Facility in IDR/UPM.

Software development for the CDF.

Feb 2010 PhD Fellowship at Instituto Universitario de Microgravedad "Ignacio Da - Sep 2011 Riva", Universidad Politécnica de Madrid (IDR/UPM).

- Support on research and industrial activities within the Institute scope, mainly structural analysis and thermal mathematical models for ESA projects.

$2008-2009 \quad$ Collaboration internship granted by Spanish Ministry of Education Escuela Técnica Superior de Ingenieros Aeronáuticos, Universidad Politécnica de Madrid, UPM)

- Subject: Parametric study of heat transfer in applicable configurations in space systems.

\section{ACADEMIC BACKGROUND}

2009-2011 Master of Science (MSc) in Aerospace Engineering at Universidad Politécnica de Madrid (UPM).

Master thesis: "Temperature stability analysis of the Fabry-Perot interferometer of PHI Instrument, on-board the Solar Orbiter mission".

2001 - $2009 \quad$ Bachelor of Science (BSc) 5-year, Aerospace Engineering at Universidad Politécnica de Madrid (UPM).

Final project: "Design and start-up of an experimental facility for measuring the average heat transfer coefficient of mechanical joints in vacuum environment". 


\section{SKILLS AND TRAINING}

Languages

- Spanish: native

- English: professional proficiency

\section{Computer skills}

- Operative systems: Windows, Mac OS.

- General: Microsoft Office

- Engineering tools: ESATAN-TMS, Patran/Nastran.

- Programming languages: Python, Fortran, VBA

\section{Communication skills}

- Experienced at delivering presentations to large audiences

- Team work as part of international groups, such as Solar Orbiter scientific payloads

- 8-year experience as Leisure Monitor in summer camps

\section{Publications}

\section{Publications, scientific and technical documents and any other publication}

G. Fernández-Rico, I. Pérez-Grande, A. Sanz-Andres, I. Torralbo, and J. Woch, Quasi-autonomous thermal model reduction for steady-state problems in space systems, Appl. Therm. Eng., 105, p. 456-466, 2016. doi: 10.1016/j.applthermaleng.2016.03.017

José Gaite, Germán Fernández-Rico. Linear Approach to the Orbiting Spacecraft Thermal Problem, Journal Of Thermophysics and Heat Transfer Vol. 26, No. 3, p. 511-522, July-Sep 2012 doi: https://arc.aiaa.org/doi/10.2514/1.T3748

\section{Work presented in conferences at national or international level}

German Fernandez-Rico, Ignacio Torralbo, Isabel Perez-Grande, Quasi-autonomous spacecraft thermal model reduction, in: $30^{\text {th }}$ Eur. Sp. Therm. Anal. Work., ESA/ESTEC, Noordwijk, The Netherlands, 2016: pp. 143-151.

https://exchange.esa.int/thermal-workshop/attachments/workshop2016/parts/QAR.pdf

I. Pérez-Grande, I. Torralbo, G. Alonso, A. Gómez-Sanjuan, G. Fernandez-Rico, Thermal Design of the ESA Solar Orbiter Instrument PHI, in: $46^{\text {th }}$ Int. Conf. Environ. Syst., American Institute of Aeronautics and Astronautics, Vienna, Austria, 2016. https://ttu-ir.tdl.org/ttu-ir/handle/2346/67685

German Fernandez-Rico, Ignacio Torralbo, Isabel Perez-Grande, Quasi-autonomous spacecraft thermal model reduction, in: $46^{\text {th }}$ Int. Conf. Environ. Syst., American Institute of Aeronautics and Astronautics, Vienna, Austria, 2016 (poster).

Ignacio Torralbo, German Fernandez-Rico, Isabel Perez-Grande, Sebastian Franchini, Gustavo Alonso, Real-time variable exchange system in a concurrent design environment, in: $6^{\text {th }}$ International Workshop on System \& Concurrent Engineering for Space Applications (SECESA), Stuttgart, 2014. 
Alejandro Gomez-SanJuan, Fermín Navarro, Javier Cubas, Felix Sorribes, German Fernandez-Rico, Gustavo Alonso, Vibrations loads in the process of designing scientific spacecraft payloads, in: $11^{\text {th }}$ International Conference on Vibration Problems, Lisbon.

Fermín Navarro, Alejandro Gomez-SanJuan, Isabel Perez-Grande, German Fernandez-Rico, Diseño preliminar térmico y estructural del instrumento NOMAD de ExoMars, in: XIX Congreso Nacional de Ingeniería Mecánica, Castellón, 2012.

G. Fernández-Rico, I. Torralbo, G. Alonso, I. Pérez-Grande, A. Martínez-Muelas, Integration of the Concurrent Design Facility in the research and educational activities at IDR/UPM, in: $5^{\text {th }}$ International Workshop on System \& Concurrent Engineering for Space Applications, Lisbon, 2012.

German Fernandez-Rico, Isabel Perez-Grande, Angel Sanz-Andres, Thermal modelling of the $\mathrm{SO} / \mathrm{PHI}$ Instrument, in: Desarrollo de instrumentación espacial. Perspectivas y estrategia para la próxima década, Madrid, 2011 (poster).

German Fernandez-Rico, Isabel Perez-Grande, Diseño térmico preliminar del Instrumento PHI de Solar Orbiter, in: VII Congreso Nacional de Ingeniería Termodinámica, Bilbao, 2011.

Germán Fernández-Rico, Isabel Perez-Grande, Ángel Sanz-Andrés, Peter Barthol, in: $20^{\text {th }}$ Symposium on European Rocket and Balloon Programmes and Related Research, Hyères, 2011, pp. 403-406. 